\title{
Indium growth and island height control on Si submonolayer phases
}

by

\section{Jizhou Chen}

\author{
A dissertation submitted to the graduate faculty \\ in partial fulfillment of the requirements for the degree of \\ DOCTOR OF PHILOSOPHY
}

\author{
Major: Condensed Matter Physics \\ Program of Study Committee: \\ Michael C. Tringides, Major Professor \\ Alan I. Goldman \\ Costas M. Soukoulis \\ Kerry L. Whisnant \\ Iver E. Anderson
}

Iowa State University

Ames, Iowa

2009

Copyright (C) Jizhou Chen, 2009. All rights reserved. 
UMI Number: 3355208

\title{
INFORMATION TO USERS
}

The quality of this reproduction is dependent upon the quality of the copy submitted. Broken or indistinct print, colored or poor quality illustrations and photographs, print bleed-through, substandard margins, and improper alignment can adversely affect reproduction.

In the unlikely event that the author did not send a complete manuscript and there are missing pages, these will be noted. Also, if unauthorized copyright material had to be removed, a note will indicate the deletion.

\section{UMI}

UMI Microform 3355208

Copyright 2009 by ProQuest LLC

All rights reserved. This microform edition is protected against unauthorized copying under Title 17, United States Code.

\author{
ProQuest LLC \\ 789 East Eisenhower Parkway \\ P.O. Box 1346 \\ Ann Arbor, Ml 48106-1346
}




\section{TABLE OF CONTENTS}

CHAPTER 1: INTRODUCTION

CHAPTER 2: EXPERIMENTAL DETAILS 19

CHAPTER 3: UNUSUAL THERMAL STABILITY OF QUANTUM SIZE EFEECT PB ISLANDS GROWN ON SI(111)-IN(4x1)

CHAPTER 4: CRYSTALLOGRAPHIC PHASE TRANSITION AND ISLAND HEIGHT SELECTION IN IN/SI(111) GROWTH

CHAPTER 5: THE EXTRAORDINARY HIGH MOBILITY OF INDIUM ATOM ON SI(111)-PB- $\alpha-\sqrt{3} \times \sqrt{ } 3$ SURFACE

CHAPTER 6: HOW INDIUM GROWS ON THE INDIUM SUBMONOLAYER PHASES ON SI(111) - DO WE NEED TO GROW ON THE ALPHA PHASE TO GET THE BEST QSE ISLANDS?

CHAPTER 7: CONCLUSIONS 104 ACKNOWLEDGMENTS 


\section{CHAPTER 1: INTRODUCTION}

Nanotechnology refers any technique that involves about object with nanoscale $\left(10^{-9}\right.$ m) or even smaller. It has become more and more important in recently years and has changed our world dramatically. Most of modern electronic devices today should thanks to the miniaturizing driven by development of nanotechnology. Recent years, more and more governments are investing huge amount of money in research related to nanotechnology.

There are two major reasons that nanostructure is so fascinate. The first one is the miniaturizing. It is obvious that if we can make products smaller without losing the features, we can save the cost and increase the performance dramatically. For an example, the first computer in the world, ENIAC, which occupied several rooms, is less powerful than the cheapest calculator today. Today's chips with sizes of less than half an inch contain millions of basic units. All these should thank to the development of nanotechnology.

The other reason is that when we come to nanoscale, there are many new effects due to the quantum effect which can't be found in large systems. For an example, quantum dots (QDs) are systems which sizes are below $1 \mu \mathrm{m}\left(10^{-6} \mathrm{~m}\right)$ and restricted in three dimensions. There are many interesting quantum effects in QDs, including discrete energy levels, and interdot coupling. Due to these properties and their small sizes, QDs have varies potential

applications such as quantum computing ${ }^{[1]}$, probe $^{[2]}$, light emitting device ${ }^{[3]}$, solar cells ${ }^{[4]}$, and $\operatorname{laser}^{[5]}$.

To meet the requirement of the nanoelectrical applications, the QDs must be grown highly uniformly because their property is highly dependent on their sizes. The major methods to grow uniform QDs include epitaxial, and lithograph. Lithography is a process to make patterns on a thin film by selectively removing certain parts of the film. Using this method, people have good control over size, location and spacing of QDs. For an example, the Extreme ultraviolet lithography (EUVL) have a wave length of $13.4 \mathrm{~nm}$ so it can curve on the surface of an sample to make structure as small as the order of $10 \mathrm{~nm} .{ }^{[6]}$ however, lithograph usually causes permanent damages to the surface and in many cases the QDs are damaged during the lithograph and therefore result in high percentage of defects. 
To avoid this, heteroepitaxy is another choice. Heteroepitaxy refers to deposit a monocrystalline film on to surface of another monocrystalline substrate. During heteroepitaxy, due to the difference of the two materials, strain is introduced. In many systems it is energetically favorable to have one or more strained layers of the overlayer separating the substrate and the islands, resulting in a hybrid growth mode usually referred to as the Stranski-Kratonov (SK, fig.2) growth mode. Self-assembled SK growth has less defects but it is much harder to control the size and position of the islands than lithograph. ${ }^{[15]}$ There are only a few parameters can be changed to control the growth, including temperature, flux rate that atoms are depositing, total amount of deposition, deposition time and the component of substrates, etc.
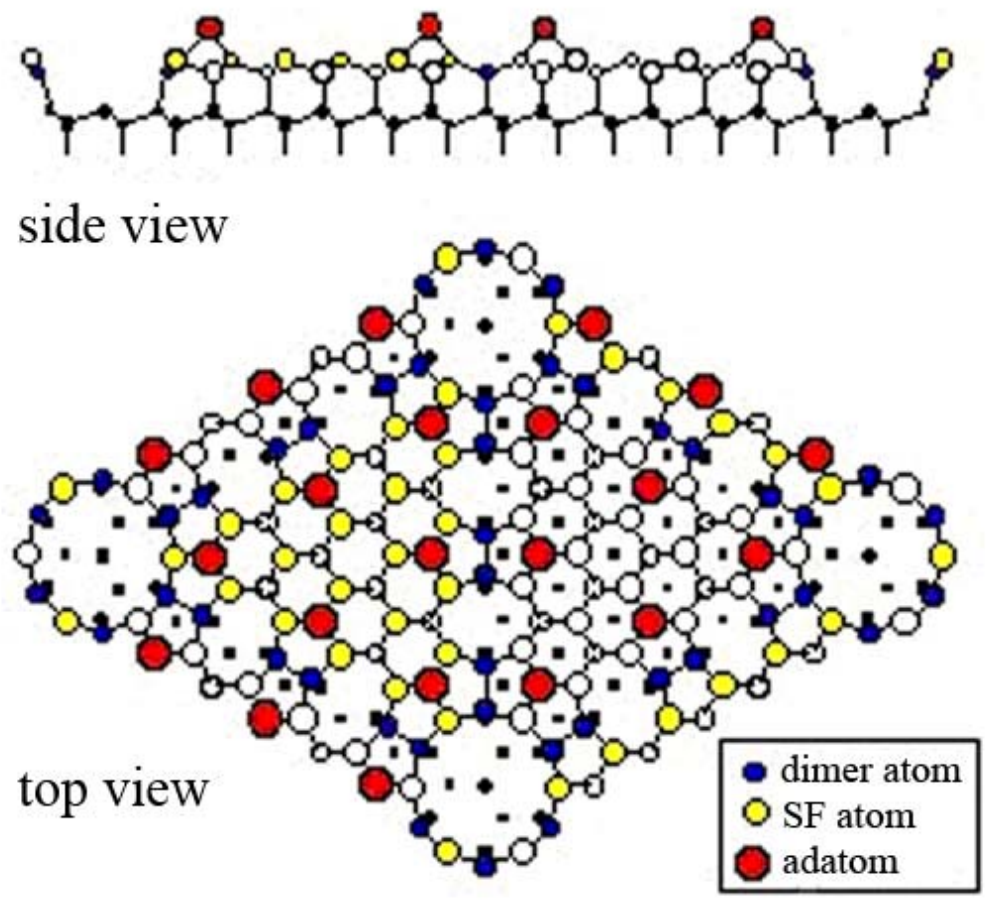

\section{Unit cell of Si(111)-7x7 DAS structure}

Figure $1 \mathrm{Si}(111) 7 \times 7$ reconstruction, taken from [6] (DAS is the acronym for dimer, adatom, stacking fault)

Many researches have been done to improve the uniform of QDs in heteroepitaxy, but it is still a long way from perfect controls. To make the QDs more uniform, some methods can be used. One is to combine lithography and heteroepitaxy. One can produce periodical defects using lithography and then grow self-assembled islands on top. For an example, for 
the growth of GaAs, people can create uniformly spaced defects (such as holes) on the GaAs substrate. The defects can serve as cores to improve the uniformity of QDs. ${ }^{[8]}$

Another way is to grow islands on patterned substrates. If a crystal is clipped along a certain plane, the atoms at the surface will reconstruct to minimize the surface energy, therefore different pattern can be produced on the surface, depend on the environment. One of the most famous structures is $\operatorname{Si}(111)-7 \times 7$ reconstruction, which is found for decades but its exact structure are not known until the birth of STM. Fig.1 shows the structure of Si (111)-7x7 reconstruction. The periodic diamond structure in the bulk was replaced by the reconstruction shown in the figure to minimize the surface energy. This structure is very important because it is the starting point of most of our experiments. Usually we can change the surface by make some depositions and/or change the temperature and the different morphology will have different ability to grow uniform islands. Therefore it is important to control the surface to find a "good" pattern that is suit for self-assembled growth.

There are many techniques to study the property of a surface, including scanning tunneling microscope (STM), which measures the quantum tunneling current between a tip and a sample to acquire information of the sample's surface, Atomic Force Microscopy (AFM), which detects the force between the tip and the sample to acquire the properties of surface, Scanning Electron Microscopy (SEM), which scans a small area of a sample to get good resolution, Transmission Electron Microscopy (TEM), which studies the sample by injecting a beam of electrons into a thin sample and observe the diffracted electrons, Low Energy Electron Microscopy (LEEM), which uses electrons instead of photons to image the sample's surface, ballistic electron emission microscopy (BEEM), which makes a beam of electrons incident from a STM tip to the surface, electrostatic force microscope (EFM), which is a special AFM that can scan without touching the sample, force modulation microscopy (FMM), magnetic force microscopy (MFM), which use magnetic materials in AFM so that magnetic forces can be detected, Piezo Force Microscopy (PFM), which uses piezo to control the tip in an AFM, etc. The instruments above provide real space picture of a surface. There are also instruments obtaining information from diffraction, these techniques includes Low Energy Electron Diffraction (LEED), which studies the structure of a crystal from the diffraction pattern from the low-energy electron beam, Surface X-ray Diffraction 
(XRD), which uses X-ray diffraction, Reflection High Energy Electron Diffraction (RHEED), which uses electrons with higher energy, Neutron diffraction (ND), which uses neutron beams, etc. Usually if we combine the two types of techniques, we can obtain both information of real space and reciprocal space. The instruments we used are STM and LEED.

STM has almost become a must have in recent research on surface physics. It provides the best real space resolution in all known instruments. LEED is a very common technique used to analysis structure of 2D or 3D crystal. Spot profile analysis LEED (SPALEED) is a special LEED which can give higher resolution in reciprocal space $(0.3 \% \mathrm{BZ})$ compared to conventional LEED, thanks to the designation of the octopole field that can control the angle of diffracted electrons. More detail descriptions are presented in Chap.2. The combination of STM and SPA-LEED can give information in both real space and reciprocal space and therefore is a very powerful tool in research in surface physics.

Usually QDs or islands' growth has a few different manners. If the deposited atoms prefer to bind to the substrate, we will see layer-by-layer growth. The atoms will fill the full layer before the next layer appearing. If the atoms prefer to bind each other, then the atoms will form islands before the first layer is fully filled. This mode is called Volmer-Weber(VW) growth. Sometimes the two modes will mix, i.e. there are complete layers as well as islands. This mode is often referred as Stanski-Kratonov(SK) mode. In either VW mode or SK mode, the islands have terrace shapes and the edges of islands have some slope. In some special condition, the atoms don't grow in any of the above three mode, they form uniform height, flat-top islands and the sides of the islands are almost perpendicular to the surface plane (Fig.2). This is due to so-called quantum size effects (QSE). 


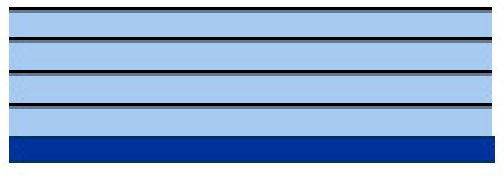

Layer by layer

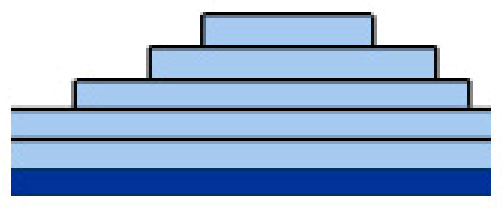

Stranski-Kratonov (SK)

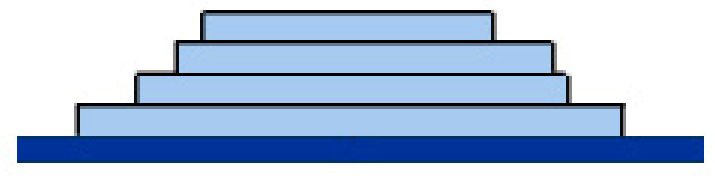

Volmer-Weber(VW)

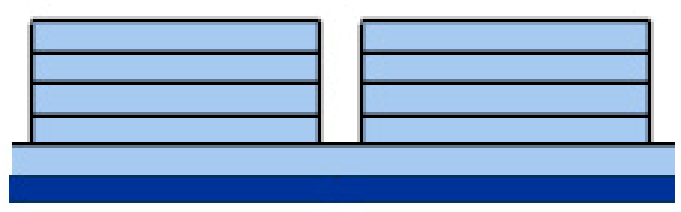

QSE electronic

\section{Figure 2 Illustration for different growth modes}

When the film thickness approaches to nanoscale, quantum confinement has a major impact on the system's physical properties. The itinerary electrons in metal films are confined in the $z$ direction perpendicular to the film surface, resulting in discrete energy levels associated with so-called quantum well (QW) states. Therefore the number of layers will significantly affect the energy level of the electrons and the surface energy will be highly dependent on the thickness of the film. Some thickness will be energetic favored and some will be unfavored. The result is that the islands will prefer to stay in certain preferred heights and therefore form uniformly height islands in the preferred heights.

For s-p metals, the QW energy levels are often be described by the quantization condition as

$$
2 \mathrm{k}(\varepsilon) \mathrm{Nd}+2 \Phi(\varepsilon)=2 \mathrm{n} \pi
$$

where $\mathrm{k}$ is the wave vector for energy $\varepsilon, \mathrm{N}$ is the number of layers, $\mathrm{d}$ is the interlayer spacing, $\Phi$ is a phase shift parameter depending on energy, $\mathrm{n}$ is an integer equal or larger than 0 . From the equation we can calculate the periodicity of QW energy level cross the Fermi level, $\Delta \mathrm{N}=\pi /\left(\mathrm{k}_{\mathrm{f}} \mathrm{d}\right)$. For an example, for $\mathrm{Pb}, \mathrm{k}_{\mathrm{f}}=0.5 \AA^{-1}, \mathrm{~d}=2.86 \AA$, therefore $\Delta \mathrm{N}=2.2^{[10]}$. This means that for $\mathrm{Pb}$ the preferred height appears every two layers. 


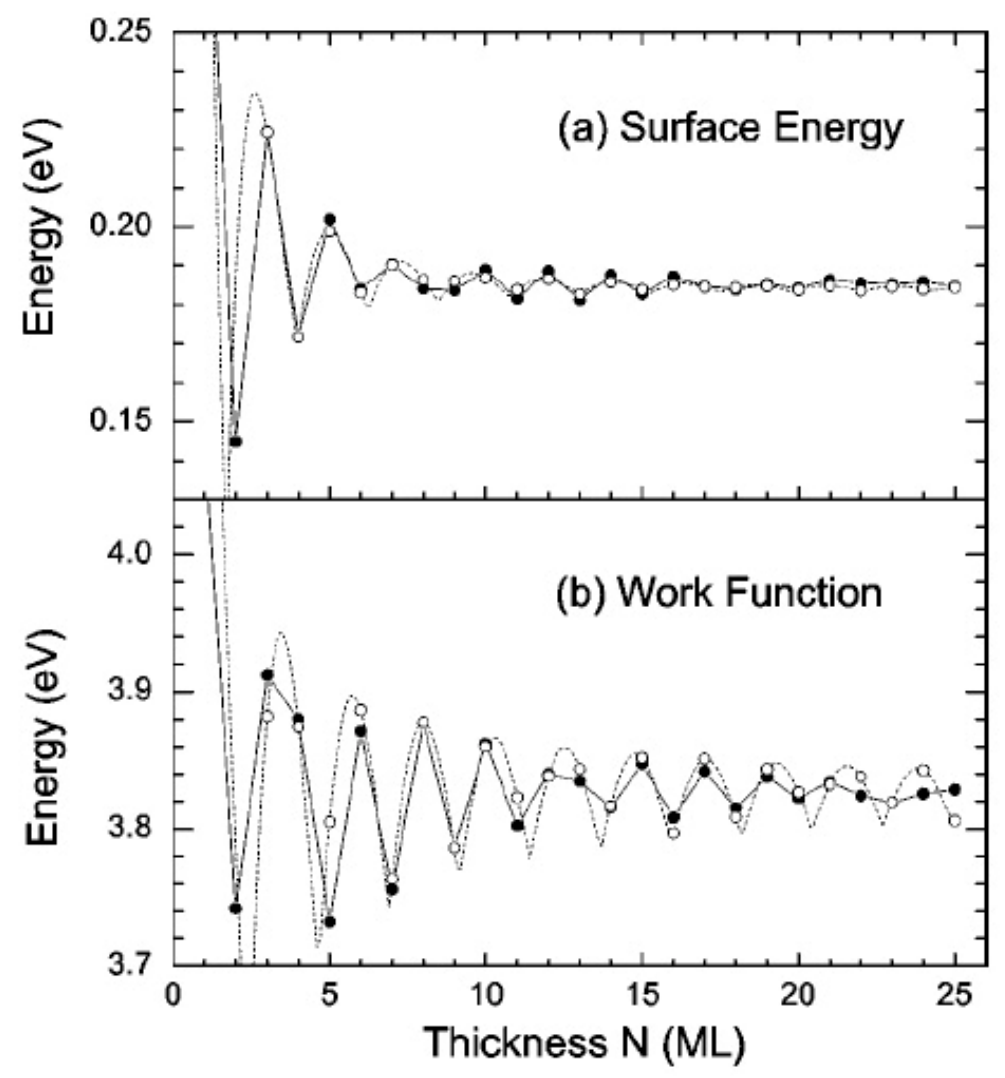

Figure 3 surface energy per $1 \times 1$ unit and work function of $\mathrm{Pb}(111)$ thin films as a function of thickness. Taken from [10]

This analysis suggests that oscillations exist in size-dependent property of the film.

Fig. 3 shows the surface energy and work function dependent on the layer thickness. The surface energy in Fig.3 is defined as one-half of the energy difference between the film and the bulk with the same number of atoms, including the proper subtraction of a term linear in $\mathrm{N}$. The work function is the energy need to remove an electron from the surface. We can clearly see that the oscillation of both surface energy and work function over layer thickness. Both the surface energy and the work function will be at minimum every $2 \mathrm{ML}$. The layer with a low surface energy typically has a surface energy much lower than the Fermi energy and thus is energetic favored. 


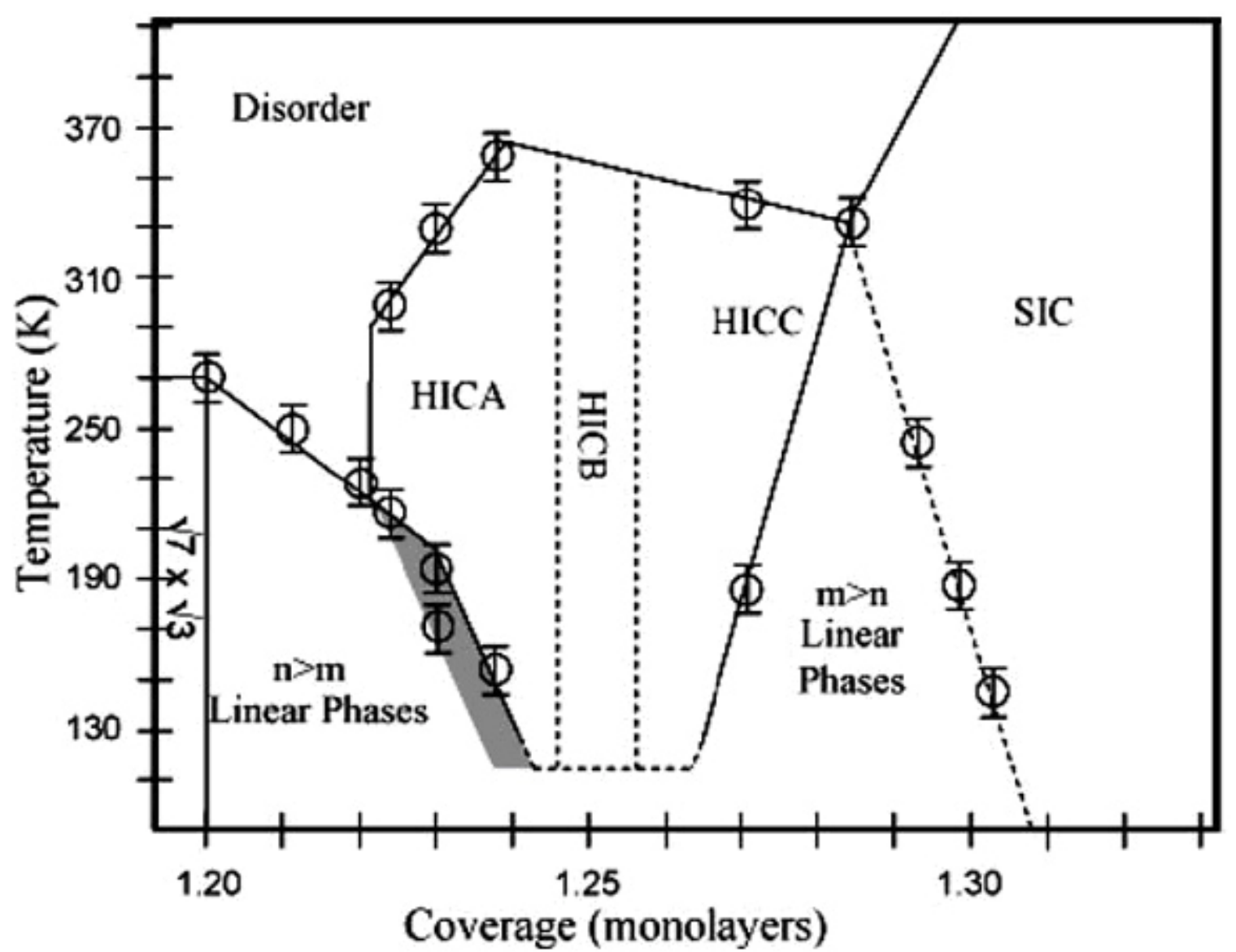

Figure 4 diagram of $\mathrm{Pb}$ phases on $\mathrm{Si}(111)$ surface. Taken from [14]

$\mathrm{Pb} / \mathrm{Si}$ (111) interface is one of the most explored metal-semiconductor interfaces and still draws many people's interests. One reason is that $\mathrm{Pb}$ is not mixable with $\mathrm{Si}$ although they are both group IV elements. What's more, $\mathrm{Pb}$ has low melting point and high vapor pressure therefore it is fit for MBE (Molecular Beam epitaxy, see Chp.2 for detail) growth. Another reason is that $\mathrm{Pb} / \mathrm{Si}(111)$ system has a rich phase diagram(Fig.4). At different coverage and temperature conditions, there are many different structures $(\sqrt{7} \mathrm{x} \sqrt{3}$, hexagonal phase, Devil's staircase phases, etc). Besides above reasons, one of the most important reasons is that strong height preference of $\mathrm{Pb}$ islands due to QSE is observed, and on different $\mathrm{Pb}$ phases the $\mathrm{Pb}$ islands have different preferred heights, which shows the potential to height control.

The $\mathrm{Pb}$ QSE islands show the possibility to control the growth of self-assemble nanostructure in height, and therefore there have been extensive studies on it. Several different techniques are used to study QSE system, including STM, LEED, XRD, Angel- 
resolved Photoemission spectroscopy (ARPES), etc. Using STM one can directly observe the morphology of films and islands, using LEED, one can analysis the dependence of the shape (00) spot on electron energy ( $\mathrm{g}$-s curve, see detailed description in chp.2) to deduce the height of the islands. In ARPES, one can yields information about the occupied states directly. Using these techniques, the $\mathrm{Pb} / \mathrm{Si}$ system has been carefully studied by many groups, e.g., (111) $\mathrm{Pb}$ islands ${ }^{[11]}$, (100) $\mathrm{Pb}$ films ${ }^{[12]}$, $\mathrm{Cu}$ islands grown on $\mathrm{Pb}$ film and influenced by the QSE in the $\mathrm{Pb}$ film ${ }^{[13]}$, etc.

Our group have done a series of works in studying the QSE on $\mathrm{Pb}$ islands on $\mathrm{Si}(111)$ substrate and related field. We first reported the uniform 7-layer height islands grown on top of $\mathrm{Si}(111)-7 \times 7$ reconstruction induced by QSE ${ }^{[9]}$. Fig.5 is an STM picture taken from $\mathrm{Pb}$ islands growth on $\mathrm{Si}(111)-7 \times 7$ surface. The size of the STM image is $200 \times 200 \mathrm{~nm}^{2}$, the temperature is $200 \mathrm{~K}$ and the coverage of $\mathrm{Pb}$ is 3.3 Monolayers (ML). Fig.5(a) shows that most of the $\mathrm{Pb}$ islands have uniform height and flat-top shape, and they are typical QSE islands illustrated in Fig.2. Fig 5(b) is the histogram, which gives the frequency distribution of height of $\mathrm{Pb}$ on the surface. It confirms the uniform of islands height: 6.7(7) ML is the most preferred height on the surface. Other observed heights include 3.8(4), 4.7(5) ML but their frequencies are at least one order lower. The height is not integer because of the existence of wetting layer on Si 7x7 surface. Fig.6 shows the g(s) curve taken from SPALEED for the 7-layer islands. The 7-fold oscillation clearly shows that the dominant height of $\mathrm{Pb}$ islands is seven. For more information about $\mathrm{g}(\mathrm{s})$ curve see chap.2.

7-layer $\mathrm{Pb}$ islands are good examples of QSE islands, but 7 is not the only possible preferred height. Further study shows that the preferred island height can be other numbers, depending on the $\mathrm{Pb}$ substrate where $\mathrm{Pb}$ islands grow on. For an example, $\mathrm{Pb}-\alpha-\sqrt{3} \mathrm{x} \sqrt{3}$ phase on $\mathrm{Si}(111)$ surface is a single layer of $\mathrm{Pb}$ on $\mathrm{Si}(111)$ surface. It removes the $\mathrm{Si}(111)-7 \mathrm{x} 7$ reconstruction and reestablishes the bulk structure (i.e., the Si atoms under the $\alpha$-phase are $1 \mathrm{x} 1$ structure) and forms a film with $\sqrt{3} \mathrm{x} \sqrt{3}$ unit cell that is smoother than the $7 \mathrm{x} 7$ reconstruction and suit for further deposition. In each $\alpha-\sqrt{3} \times \sqrt{3}$ unit cell there are four $\mathrm{Pb}$ atoms and the size of $\sqrt{3} \times \sqrt{3}$ unit cell is 3 times of Si $1 \times 1$ unit cell, so the coverage is $4 / 3$ ML. (See chap. 2 for more detail decription for $\mathrm{Pb}-\alpha-\sqrt{3} \times \sqrt{3}$ phase) When we grow $\mathrm{Pb}$ islands on $\mathrm{Pb}$ alpha- $\sqrt{3} \mathrm{x} \sqrt{3}$-phase, the first stable height is 3 layers from silicon (i.e. 2 layers from the 
wetting layer). The next stable height is 6 layers and after that, the islands grow in a bi-layer manner.

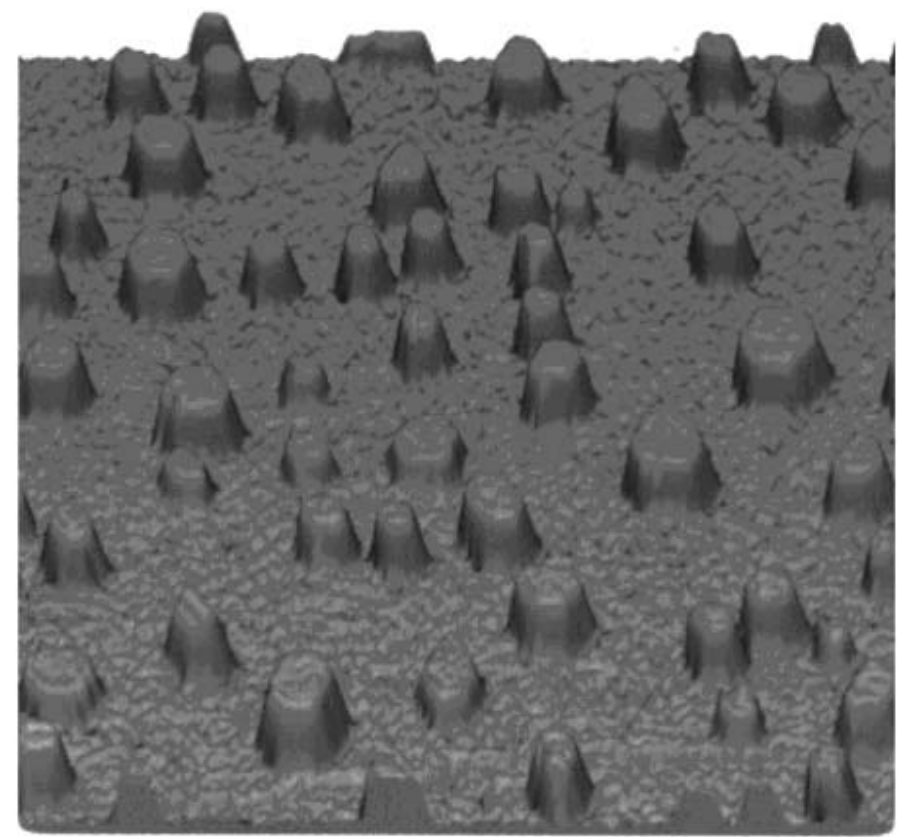

(a)

\section{histogram}

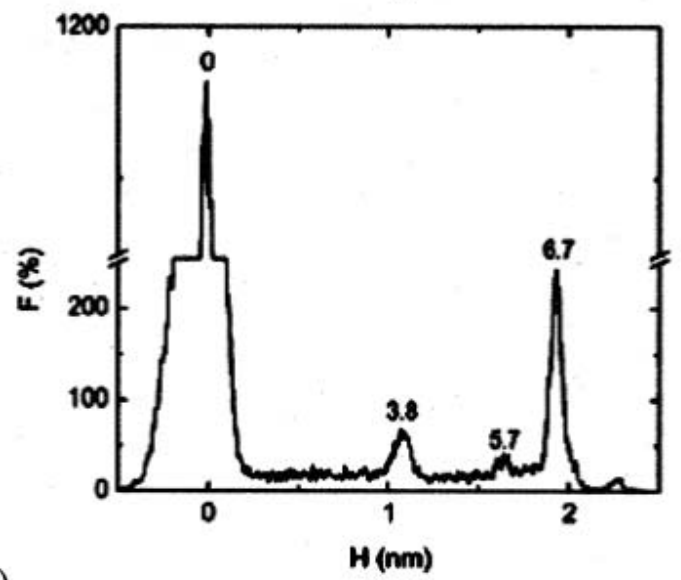

(b)

Figure 5 200nmx200nm STM picture of $\mathrm{Pb}$ islands on $\mathrm{Si}(111) 7 \times 7$ surface and the histogram. Taken from [9]

An experiment is performed to study the QSE effect on Pb alpha- $\sqrt{3} x \sqrt{3}$-phase ${ }^{[16]}$. Initially, 1.6ML of $\mathrm{Pb}$ was put on the top of $\mathrm{Pb}-\alpha-\sqrt{3} \mathrm{x} \sqrt{3}$ phase at $\mathrm{T}=180 \mathrm{~K}$ and most of the surface is covered by 3 -layer (count from silicon) islands. After additional 1.3ML of $\mathrm{Pb}$ was deposited, most of the islands grow to their next stable height, 6 layers(Fig.7). It is found that 
less than $15 \%$ of the 6-layer islands are covered by $\mathrm{Pb}$ atoms, while the corresponding number for stable layers covering unstable height(e.g. 5 layer) islands is $85 \%$. From this we can tell the $\mathrm{Pb}$ atoms prefer to leave the top of stable islands and go to the top of unstable islands, which results in the conversion from unstable islands to stable ones. In other words, the $\mathrm{Pb}$ atoms on the stable 6-layer $\mathrm{Pb}$ islands tend to diffuse to lower layer to increase the lateral dimension of the islands, instead of building the next layer. On the other hand, the $\mathrm{Pb}$ atoms on the unstable layers tend to stay on top of the islands to form the next layer to reach the stable height. Therefore it can be implied that stable $\mathrm{Pb}$ islands have a lower Ehrlich-Schwoebel (E-S) barrier at the edge of the islands, while unstable islands have a higher E-S barrier at the edge. This experiment shows the QSE in kinetic growth.
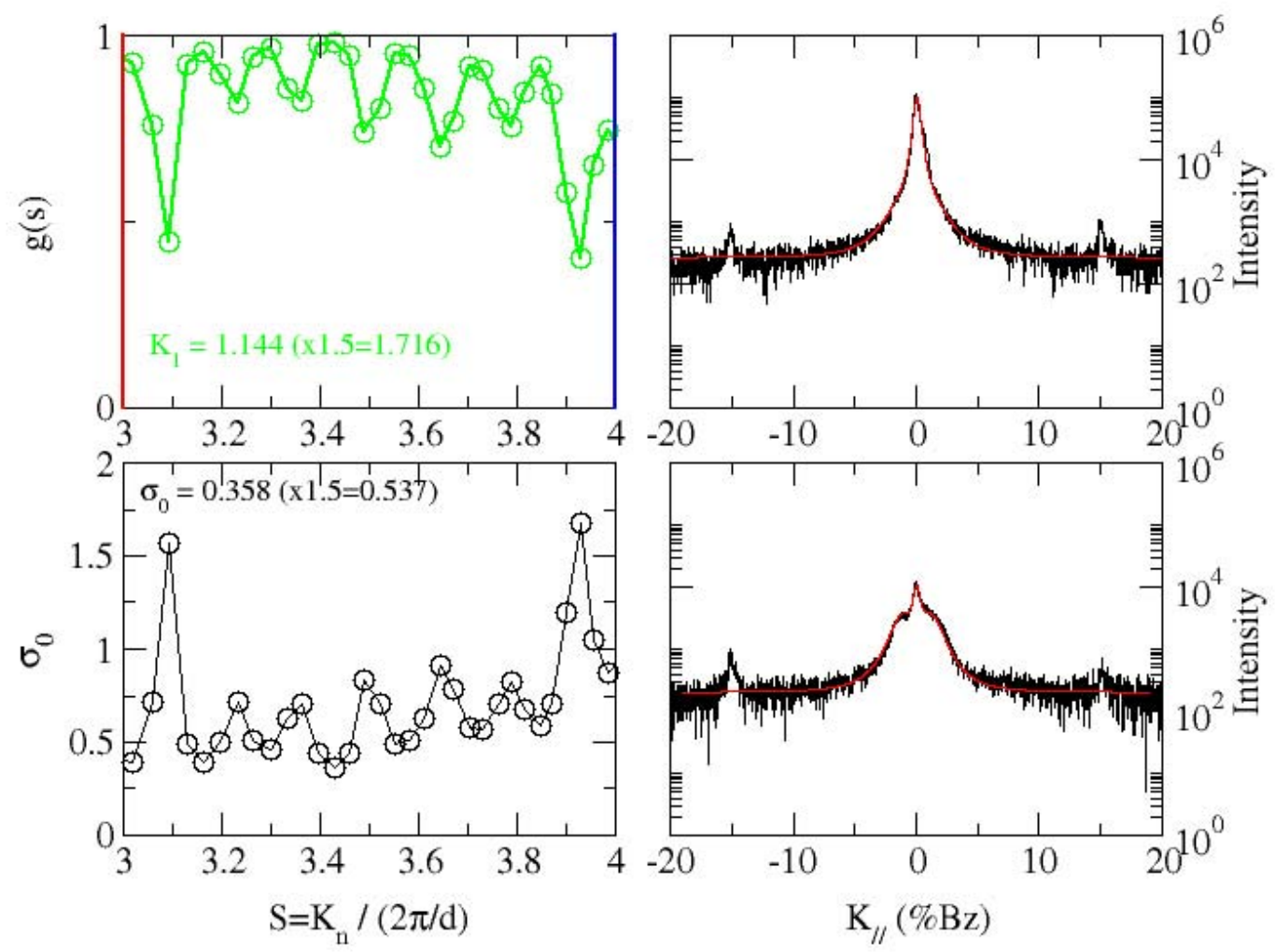

Figure $6 \mathrm{~g}(\mathrm{~s})$ curve for 7-layer $\mathrm{Pb}$ islands on $\mathrm{Si}(111) 7 \times 7$ phase. 

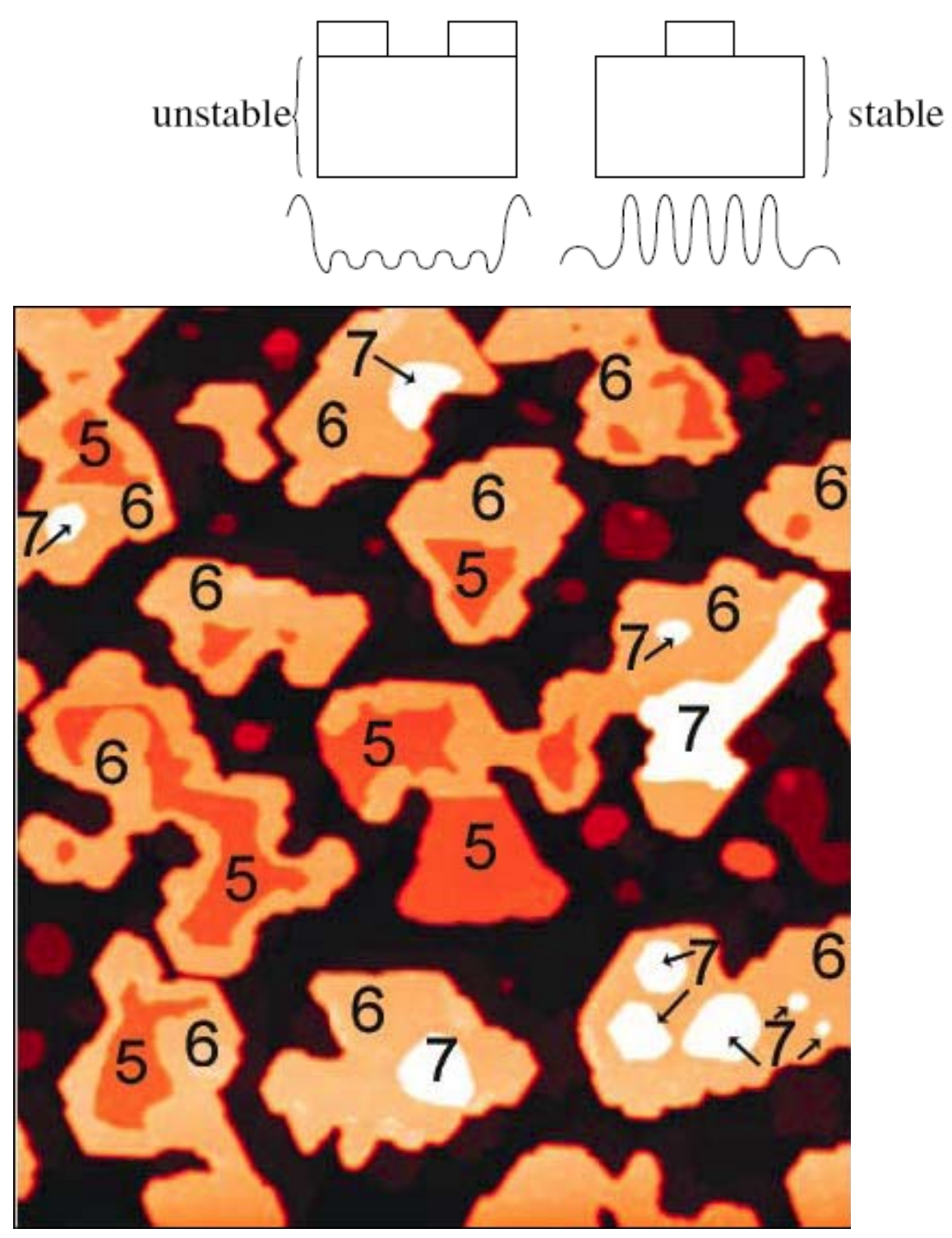

Figure 7 The upper part shows the different growth type on stable and unstable islands. The lower parts shows an 330nm x 370nm images with total Pb coverage of $2.9 \mathrm{ML}$ on $\mathrm{Pb}$ alpha- $\sqrt{3} \times \sqrt{3}$-phase at $T=180 \mathrm{~K}$.

It is observed that all the 6-layer islands complete their top by growing from the perimeter to the center with almost constant width, while the next layer's growth starts with small compact islands in the center. It can be seen in fig. 7. 


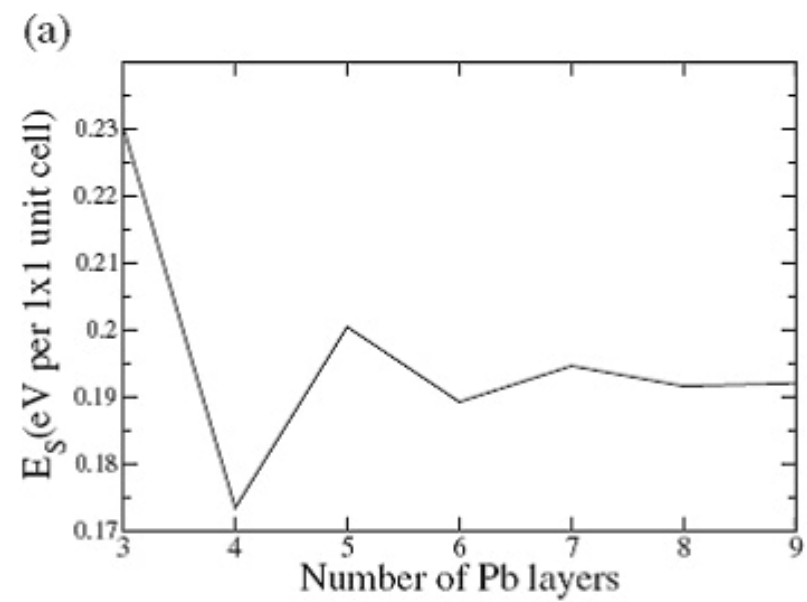

(b)

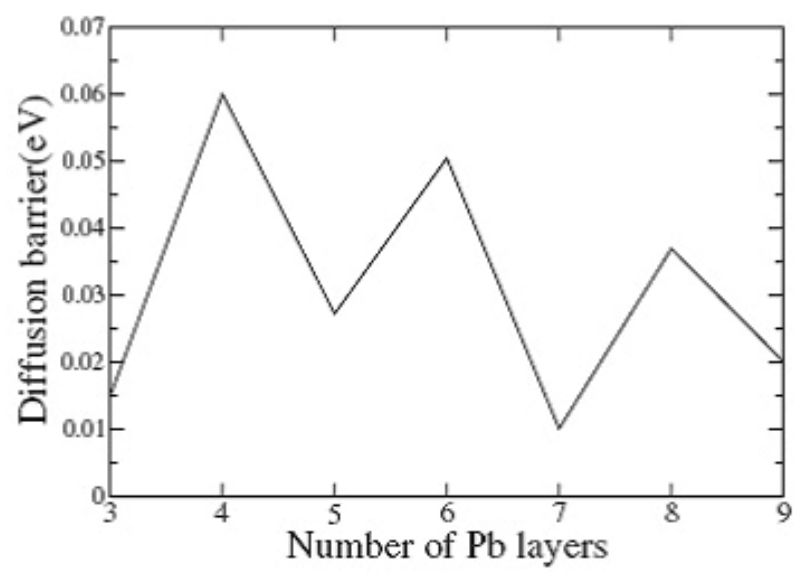

Figure 8 (a) The surface energy per $1 \times 1$ unit cell of a freestanding Pb film from 3 to 9 atomic layers; (b) The diffusion barrier of a $\mathrm{Pb}$ adatom on a 3- to 9-layer $\mathrm{Pb}$ film. Taken from [16]

To understand the unusual growth morphology, we need to study the diffusion of $\mathrm{Pb}$ adatoms as a function of thickness using first-principle total energy calculation. ${ }^{[16]}$ The calculation is carried out using the VAST program ${ }^{[17,18]}$ based on the first-principles densityfunctional theory ${ }^{[19]}$. The surface energy is calculated using formula $E s=\left(E_{\text {film }}-n \times E_{\mathrm{Pb}}\right) / 2$, where $E_{\text {film }}$ is the total energy of the $\mathrm{Pb}$ film, $n$ is the number of $\mathrm{Pb}$ atoms in the system, and $E_{\mathrm{Pb}}$ is the energy of a $\mathrm{Pb}$ atom in its crystalline fcc structure. The result shown in Fig.8 clearly displays the bi-layer oscillations of surface energy from 3-layer to 9-layer due to QSE and it agrees well with the result in Fig.3.

The unusual growth morphology, especially the difference between nucleation on top of stable versus unstable islands, for $\mathrm{Pb}$ islands on $\mathrm{Si}(111)$ observed in the STM experiment can be attributed to the large oscillation in the diffusion barrier between the stable and 
unstable $\mathrm{Pb}$ films. The unstable islands (7 layers) have a lower barrier while the stable islands (6 layers) have a higher barrier.

SPA-LEED experiments are consistent with STM. We can take scan of (00) spots at different energies and make the $g(s)$ curve, from the $g(s)$ curve we can deduce the dominate islands height on the surface. (See Chp.2 for more details about g(s) curve)

Monte Carlo simulation is also performed to simulate the bi-layer growth of $\mathrm{Pb}$ islands ${ }^{[20]}$. Monte Carlo simulation is a computational algorithm by repeating random sampling to get results base on a certain model. To make a correct simulation, it is important to choose proper potential-energy surface and barriers. Fig.9 shows the simulation result that showing bi-layer growth and the atoms grow from the edge and form rings, which are consistent with both experiments and calculations.

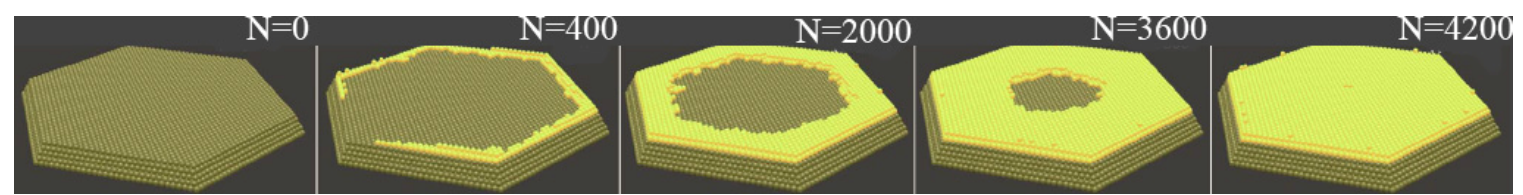

Figure 9 bi-layer growth of Pb islalnds by Monte Carlo simulations. Taken from [18]

Different experiments and first-principle calculations have shown that the bi-layer stability of $\mathrm{Pb}$ islands is due to the QSE. The island stability was observed directly from oscillations of the position of the highest occupied band (HOB) with respect to the Fermi level, i.e., heights with HOB far from the Fermi level correspond to stable heights, since the confined electron energy is lower. However, this oscillatory dependence of the energy level positions with respect to the Fermi level has been also observed in other systems (e.g. $\left.\mathrm{Cu} / \mathrm{Co}(100),{ }^{[20]}\right)$ where QSE are also present. In these systems, the position of the HOB with respect to the Fermi level also oscillates with thickness, therefore we may ask why the preferred heights have not been observed in these systems as well. One possible reason is kinetics, i.e., some unusually mechanism which is unique in $\mathrm{Pb} / \mathrm{Si}(111)$ system that enables the system to find the QSE-driven energy minima within the duration of the experiment.

A study of $\mathrm{Pb}$ islands coarsening is made to attempt to study the kinetics of $\mathrm{Pb}$ island growth. ${ }^{[21]}$ It is found that the wetting layer between $\mathrm{Pb}$ islands plays an important role. After a small deposition (0.25ML) to $\mathrm{Pb}$ islands on $\mathrm{Si}(111) 7 \times 7$ at $180 \mathrm{~K}$, the 4-layer islands those have rings of single layer growing to the next stable 5-layer islands. We notice that the amount of $\mathrm{Pb}$ deposited on to the islands is far less than the amount need to grow the islands, 
which means a large amount of $\mathrm{Pb}$ atom must come from the wetting layer. This also explains why the growth is much faster than expected in classical coarsening theory. It is amazing the way that the wetting layer moves: it spread within minutes along the island perimeter to form the "ring" but takes much longer time to completely fill it. This diffusion explains why the islands are completed in as short as a few minutes.

Although $\mathrm{Pb} / \mathrm{Si}(111)$ interface is very special, it is not the only one where we can see the height preference in growth of ultrathin films due to quantum size effects. Ag is found to have height preference for a long time, and we recently find that $\mathrm{In} / \mathrm{Pb}$ interface is also very interesting.

Like $\mathrm{Pb}$, In also has a rich phase diagram. Ref[22] gives a general survey of In phases, there are many phases still not fully understood. In doesn't mix with $\mathrm{Si}$, too, and like $\mathrm{Pb}$, In has a low melting point and high vapor pressure so it is also fit for MBE.

In an experiment growing $\mathrm{Pb}$ islands on $\mathrm{In} 4 \mathrm{x} 1-\mathrm{Si}(111)$ substrate, we observe that the islands are controllable not only in height by QSE, but also controllable in width due to restrain anisotropy of the $\operatorname{In}(4 \mathrm{x} 1)$ reconstruction. ${ }^{[23]}$ In $4 \times 1$ substrate is an In single layer film with unit cell 4x1(in unit of Si lattice constant). Fig.10 shows an STM images of Pb islands growing on In 4x1-Si(111) surface. These islands have not only uniform height (4 layer), but also uniform width $\left(2 \mathrm{w}_{0}\right.$ or $3 \mathrm{w}_{0}, \mathrm{w}_{0}=1.33 \mathrm{~nm}$, according to the model based on $\mathrm{x}$-ray diffraction ${ }^{[24]}$, is the distance between two $4 \times 1$ rows). Thanks to the high-resolution of STM images, the width of $\mathrm{Pb}$ islands can be easily measured. This experiment shows that by introducing the In $4 \times 1$ substrate, we can control two dimensions of the islands, instead of one dimension. It is one step closer to the final goal to control the growth in all three dimensions. 


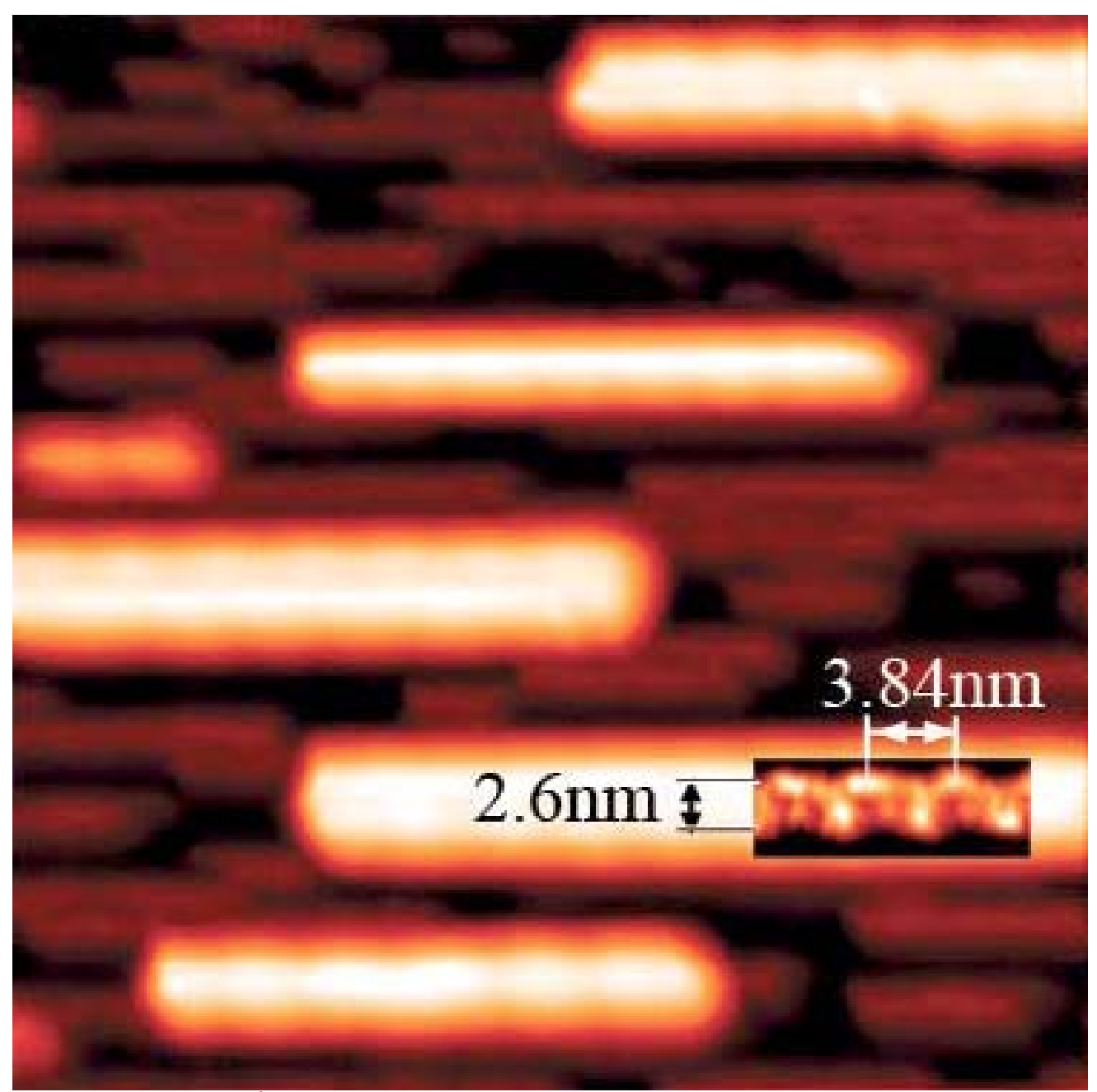

Figure $1050 \times 50 \mathrm{~nm}^{2}$ image when $2 \mathrm{ML} P b$ is deposited on In 4x1 substrate. Two-layer elongated islands of widths $\left(2 w_{0}\right)$ form with the corrugation on top. The islands height is 4 layer from the In or 2 layer from wetting layer. (the wetting layer is 2 layer-high.) Taken from [23]

Due to the discover of the $\mathrm{Pb} / \mathrm{In}(4 \mathrm{x} 1)$ system, it is worth investing more study in the $\mathrm{In}-\mathrm{Pb}$ system. In further study we find that the 4-layer $\mathrm{Pb}$ islands have an extra stability at temperature as high as room temperature ${ }^{[25]}$. Usually the $\mathrm{Pb}$ islands are metastable and exist only at low temperate $(<200 \mathrm{~K})$, but the $\mathrm{Pb}$ islands on $\mathrm{In} 4 \mathrm{x} 1$ phase remains even after $\mathrm{RT}$ overnight annealing.

We not only studied $\mathrm{Pb}$ grown on In $4 \times 1$ substrates, but In islands grown on $\mathrm{Pb}$ substrates, too. Firstly we studied the In islands grown on top of $\mathrm{Pb}$ alpha $(\sqrt{3} \mathrm{x} \sqrt{3})$ phase. QSE driven Islands height preference was observed and a face-center-cubic (FCC) to bodycenter-tetragonal (BCT) indium phase transition is seen. The In islands grow following the FCC structure of substrates at low coverage and prefer to be 4-layer high(count from wetting 
layer) due to QSE. However, the FCC structure is not energetic favored when the coverage increases, and eventually the structure of In islands convert to BCT (bulk structure of indium crystal). ${ }^{[26]}$

When we grow indium islands on $\mathrm{Pb}$ alpha phase one novel phenomenon draws our interests. After the FCC islands transferred to $\mathrm{BCT}$ islands, their growth becomes extra fast. Figure 11 shows $200 \mathrm{~nm} \times 200 \mathrm{~nm}$ STM images of In islands grown on $\mathrm{Pb}$ alpha phase at coverage $=2.5 \mathrm{ML}$ and $5 \mathrm{ML}$. The biggest island in the figure is in bet structure and other islands around it are in FCC structure. We can see between the two images the BCT islands grows significantly (from 10 layer to 28 layer ) while the FCC islands just grow a little bit in height. This unusual growth shows extra high mobility of In atoms, since the amount of In atoms required by the $\mathrm{BCT}$ island is much more than the amount deposited on to it. The $\mathrm{BCT}$ islands must like a vacuum cleaner that absorbs most indium atoms nearby from the wetting layer. In another word, many In atoms on the wetting layer goes to the farther BCT islands instead of nearby FCC islands. (Details are discussed in Chap.5)

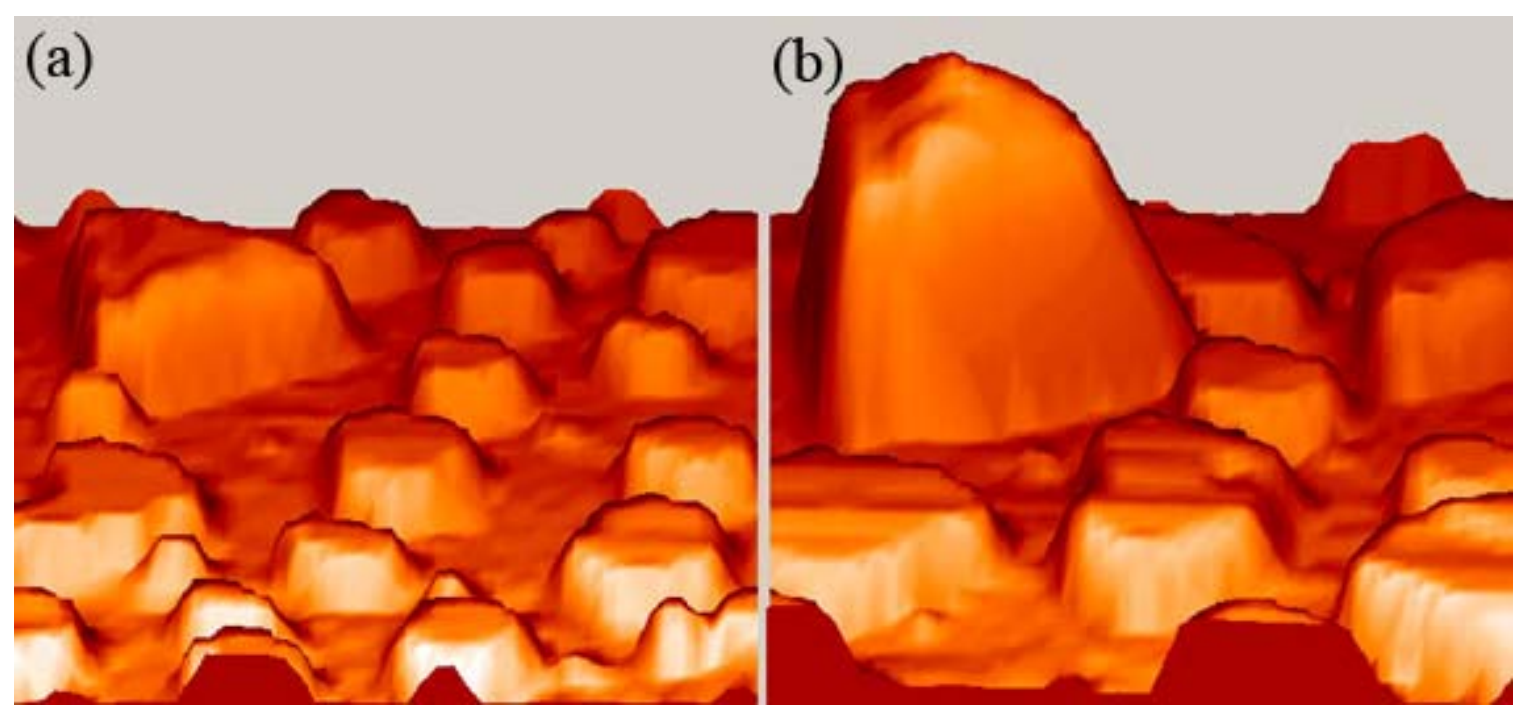

Figure 11 In islands on Pb alpha phase. (a) 2.5ML (b) 5ML image size $=200 \mathrm{~nm} \times 200 \mathrm{~nm}$. $\mathrm{T}=200 \mathrm{~K}$ It will be interesting to learn when we deposit In on In phases, what will happen.

There are a lot of studies on growth on In phases before. For an example, Bauer etc. have studied extensively the growth of In islands in different phases. They grows In on varies In phases, $(4 \mathrm{x} 1, \sqrt{3} \mathrm{x} \sqrt{3}$, etc) at Temperature between $300 \mathrm{~K}-500 \mathrm{~K}$. These low coverage phases can convert to higher coverage phases with In deposition and they have found many different In phase, i.e., $2 \times 1,1.5 \sqrt{ } 3 \times 1.5 \sqrt{ } 3,1 \times 1$ R 30 , etc. These phases are named after their unit cell. 
For an example, 1x1R30 refers to the In phase with the same unit sell as Si (there is 1x1) and rotate by $30^{\circ}$ from Si unit cell, however, there is no growth experiment done at low temperature $(<200 \mathrm{~K})$.

Therefore, it may be useful to study the In deposition in low temperature. we studied the In islands growing on varies of In substrates (In beta or $\sqrt{3} x \sqrt{3}$ phase, In $4 x 1$ phase, In $\sqrt{ } 31 x \sqrt{ } 31$, etc.) in SPA-LEED at low temperature $(<180 \mathrm{~K})$. We found most In phase will convert to In 1x1R30 and then the In 1x1R30 phase serve as a wetting layer and islands grow on top it. Surprisingly, although fcc spots show up at low coverage, we can not find bet spots when coverage increases and FCC spots fade away. Do BCT islands exist on In substrate? The answer is yes. The reason that we can't see BCT spots is BCT islands grow too fast and become extra huge in a very short time so that we can not even detect the BCT spots in diffraction. We believe that the high mobility of In islands is the main reason. We also tried to add some $\mathrm{Pb}$ to reduce the speed of diffusion of In atoms and it seems working in some degree. We didn't find height preference of In islands height by diffraction, which may also related to the mobility of In atoms.

By comparing the In growth on $\mathrm{Pb}$ and In substrate, we find that $\mathrm{Pb}$ alpha phase is very special. It is the only substrate where In islands growth have height preference due to QSE. The reason is still not clear yet, but the $\mathrm{Pb} / \mathrm{In}$ interaction may have some contribution.

This thesis primarily focuses on the In and $\mathrm{Pb}$ islands growth on the thin $\mathrm{In} / \mathrm{Pb}$ induced $\operatorname{Si}(111)$ surface. Chapter 2 is the review of the experiment setup and the two main techniques: SPA-LEED and STM. In chapter 3 we will discuss the unusual stable Pb islands on In $4 x 1$ phase. In Chapter 4 we discuss the phase transition of In islands on $\mathrm{Pb}$ alpha phase. In chapter 5 we discuss the unusual fast grow of In bet islands. In chapter 6 we discuss the In growth on several different In substrate. In the end is a brief summary.

\section{References:}

[1] G. Burkhard, et al, Phys. Rev. B 59, 2070 (1999)

[2] Maxime Dahan, et al, Science 302, 442 (2003)

[3] B. O. Dabbousi, et al, Appl. Phys. Lett. 66, 1316 (1995). 
[4] Ilan Gur, et al, Science 310, 462 (2005)

[5] M. Grundmann, Physica E 5, 167 (2000)

[6] S. Bajt, et.al, Opt. Eng. 41, 1797 (2002)

[7] http://www.ohdomari.comm.waseda.ac.jp/research/surf/stm/stm.html (org.

Takayanagi et al., J. Vac. Sci. Techn. A 3, 1502 (1985).)

[8] H. Heidenmeyer, C. Muller, O.G. Schmidt, J. Crys. Growth 261, 444 (2004)

[9] M. Hupalo, et al, Phys. Rev. B 64, 155307(2001)

[10] C. M. Wei, et al, Phys. Rev. B 66, 233408 (2002)

[11] T.L. Chan, et, al, Phy. Rev. Lett. 96, 226102 (2006)

[12] Li-Ying Ma et al., Phys. Rev. Lett. 97, 266102 (2006)

[13] C. M. Wei, et al, Phys. Rev. B 75, 195417 (2007)

[14] Stepanovsky, et al., Surf. Sci. 600, 1417 (2006)

[15] G. Costantini, A. Rastelli, C. Manzano, R. Songmuang, O. G. Schmidt, K. Kern., Appl. Phys. Lett. 85, 5673 (2004)

[16] T.Z. Chan, et al., Phy. Rev. Lett. 96 226102(2006)

[17] Vienna ab initio simulation package, Technische Universita“t Wien, 1999; G. Kresse and J. Hafner, Phys. Rev. B 47, 558 (1993)

[18] G. Kresse and J. Furthmuller, ibid. 54, 11169 (1996).

[19] P. Hohenberg and W. Kohn, Phys. Rev. 136, B864 (1964); W. Kohn and L. J. Sham, ibid. 140, A1133 (1965).

[20] Z.Kuntova, et al., Phy. Rev. B 75, 205436 (2007)

[21] M. Hupalo, et al., Phys. Rev. B 75, 235443 (2007)

[22] A. Pavlovska, et al., J. Vac. Sci. Technol. B 20,6 (2002)

[23] M. Hupalo, et al., Phys. Rev. B 73, 041405 (2006)

[24] O. Bunk et al., Phy. Rev. B 5912228 (1999)

[25] M. Yakes, et al., App. Phys. Lett. 90, 163117 (2007)

[26] J. Chen, et al. Phys. Rev. B 77, 233302 (2008) 


\section{CHAPTER 2: EXPERIMENTAL DETAILS}

Proper instruments are required to study the surface of a sample. This chapter will give a detailed description to the two instruments in our research: STM and SPA-LEED.

In order to work on surfaces of materials it is important that the conditions of the experiment do not change during the time of experiments. In a normal atmosphere a typical silicon surface will be tainted by the molecules in atmosphere in around $10^{-9}$ second. Therefore, to keep the surface conditions the same throughout the experiment it is necessary to reduce the number of particles in the air that interact with the sample by reducing the pressure at the surface of the sample. In another word, the experiments must be performed in an Ultra High Vacuum (UHV) environment. The pressure of the system during an experiment must be less than $10^{-9}$ torr ( $1 \mathrm{~atm}=760$ torr) to prevent the sample from being polluted by the atmosphere. Both the STM and SPA-LEED system are placed in UHV chambers with base pressure of $10^{-11}$ torr.

In the chamber where experiments are performed, three vacuum pumps are used to guarantee the required vacuum. The first pump is a turbo molecular pump which can reach a

pressure of $10^{-8}$ torr. To further improve the vacuum, an ion pump and a titanium sublimator are used. In an ion pump a potential difference is created across two large metal plates. Gas atoms between the plates are ionized and they are accelerated toward the edge of the plates. The titanium sublimator provides titanium atoms into the chamber. The titanium atoms are very active, they will attract many reactive particles in the air such as hydrogen and oxygen molecules. The sublimator works like a sponge and make the molecules in the air easily be removed by other pumps.

Baking is also needed to reach a vacuum as high as $10^{-11}$ torr. When a vacuum chamber is opened and exposed in air, there will be a layer of air or water accumulated on the surface. It is necessary to remove this layer by baking the system, otherwise the layer will slowly evaporate into the chamber and raising the pressure of the chamber after the chamber is closed. To bake the chamber, heating coils are wrapped all over the outside of the system, and the system is covered with aluminum foils to keep the heat in the chamber and to retain 
the uniform of temperature in the whole system during baking. Then the whole system is heated to below $200^{\circ} \mathrm{C}$. It is important to control the baking temperature. If the temperature is too low, the attaching layer can't be removed. If the temperature is too high, the heat may cause some permanent damages in the chamber. It is necessary to bake the system each time after opening the chamber in order to achieve UHV no less than $10^{-9}$ torr.

After baking, the base pressure of the chamber can reach $10^{-11}$ torr or better and experiments can be performed.

The sample in the UHV chamber is a very thin silicon slice cut in (111) plane. Even in a UHV chamber the surface of the sample will be covered by residual gas after one day. Therefore we must clean the sample each time before an experiment. The cleaning ${ }^{[1]}$ is called "flashing". First we heat the crystal to about $1250^{\circ}$ which is nearly the melting point of Si so that everything on the surface of the Si will be removed. At this stage the sample is very bright and shining, this is the reason we call the procedure "flashing". The time of flashing should not be too long, or the structure of the sample may be damaged. Usually we flash the sample for about 20 seconds, which is enough to clear the impurities on the surface in most cases. The sample temperature is then reduced to $800^{\circ} \mathrm{C}$ slowly and the temperature is kept for 2 minutes. At this temperature the Si $7 \times 7$ reconstruction is formed. After that, the temperature quickly drops to room temperature or even lower and the flashing is finished. After flashing, we can check the quality of the $7 \times 7$ reconstruction. In the SPA-LEED chamber we should observe a very sharp 7x7 diffraction patter while in the STM chamber we should get images with atomic resolution.

If the Si sample is used for the first time, it is covered with an oxide film for protection. In this case, the crystal needs to be flashed multiple times to completely remove the oxide film from the sample. The pressure must be below $10^{-9}$ torr to prevent the crystal from roughening. Once the film is removed, then typically we only need to flash the sample once to clean the surface of the sample.

After the Si sample is cleaned, we can deposit other materials onto the surface of $\mathrm{Si}$ sample. The deposition is done by a method known as molecular beam epitaxy (MBE). In the experiments in this thesis the molecular beams are controlled by a Knudsen cell (K-cell). The $\mathrm{K}$-cell is a good tool for reproducibly depositing small area thin films of atoms. The material 
(usually metals) to be deposited is heated to provide a vapor pressure in an isothermal enclosure. The vapor will effusion from an aperture in the end of the K-cell. There is a shutter at the aperture to control the opening or closing of the aperture. The deposition rate is extremely stable when the temperature of the enclosure, which is accurately controlled by a thermocouple and temperature controller, is stable. Usually after 6-10 minutes of heating, the temperature of the enclosure is stable. The shutter can then be opened for depositing.

The crystal is cooled with a water bath and other shielding devices to reduce the radiation from the surrounding environment, which would cause outgassing and raise the pressure inside the chamber. The K-cell is especially useful for materials with low melting temperature and high vapor pressure such as lead and indium. The saturated vapor pressure is dependent on the temperature, therefore the deposition rate can be controlled with great precision by adjusting the temperature of the material. After the equilibrium is reached and temperature is stable, the shutter can be open and depositions can be made.

To calibrate the flux rate of depositions, two methods are used. For $\mathrm{Pb}$ source, it can be done by preparing the $\sqrt{3} x \sqrt{3}$-beta phase and $\sqrt{7} x \sqrt{3}$ phase. $\sqrt{3} x \sqrt{3}$-beta phase is a 2 -D Pb phase which have a unit cell of $\sqrt{ } 3 \mathrm{a}_{0} \times \sqrt{ } 3 \mathrm{a}_{0}$, where $\mathrm{a}_{0}$ is the unit cell of Si structure. The height of the cell is $3 a_{0} / 2$, so its area is $\sqrt{ } 3 a_{0} *(3 / 2) a_{0}=(3 \sqrt{ } 3 / 2) a_{0}{ }^{2}$, which is 3 times of area of Si unit cell $\left((\sqrt{3} / 2) \mathrm{a}_{0}{ }^{2}\right)$. There is one $\mathrm{Pb}$ atom in each $\sqrt{3} \mathrm{x} \sqrt{ } 3$ unit cell, so its coverage is 1/3ML. The $\sqrt{7} x \sqrt{3}$ phase has a unit cell of $\sqrt{ } 7 a_{0} x \sqrt{ } 3 a_{0}$. The height of the cell is $5 a_{0} / 2$, so its area is $\sqrt{ } 3 \mathrm{a}_{0} *(5 / 2) \mathrm{a}_{0}=(5 \sqrt{ } 3 / 2) \mathrm{a}_{0}^{2}$, which is 5 times of area of the Si unit cell. There are $6 \mathrm{~Pb}$ atoms in one unit cell, so its coverage is $6 / 5=1.2 \mathrm{ML}$. Fig. 1 gives the illustration of $\mathrm{Pb}$ beta and $\mathrm{Pb} \sqrt{ } 7 \mathrm{x} \sqrt{3}$ phase in real space.

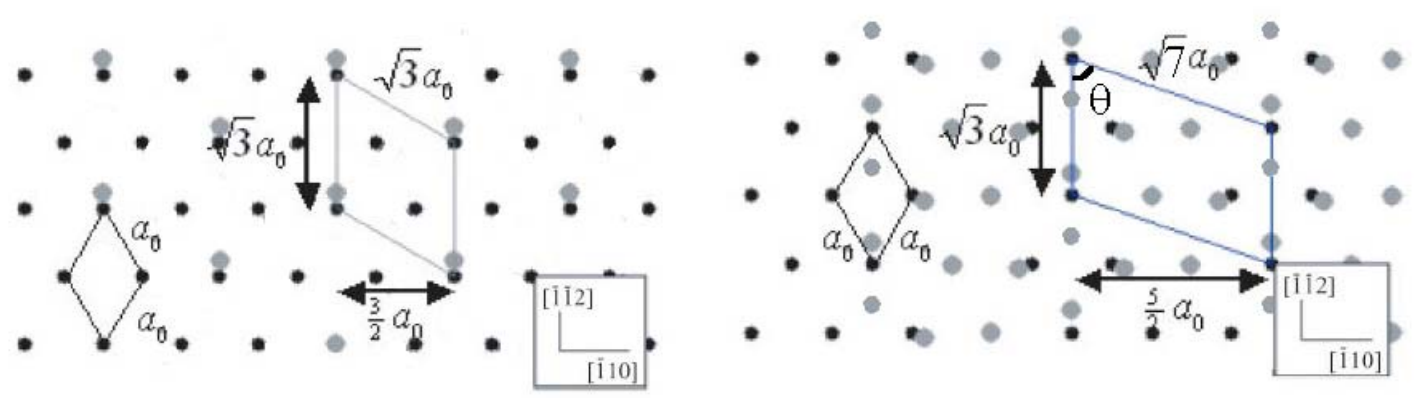

Figure 1 Illustration for $P b \sqrt{3} x \sqrt{3}$ (beta)phase and $\sqrt{7} x \sqrt{3}$ phase. Black dots are Si atoms and grey dots are $\mathrm{Pb}$ atoms. In each figure a unit cell is draw as well as a Si unit cell. 


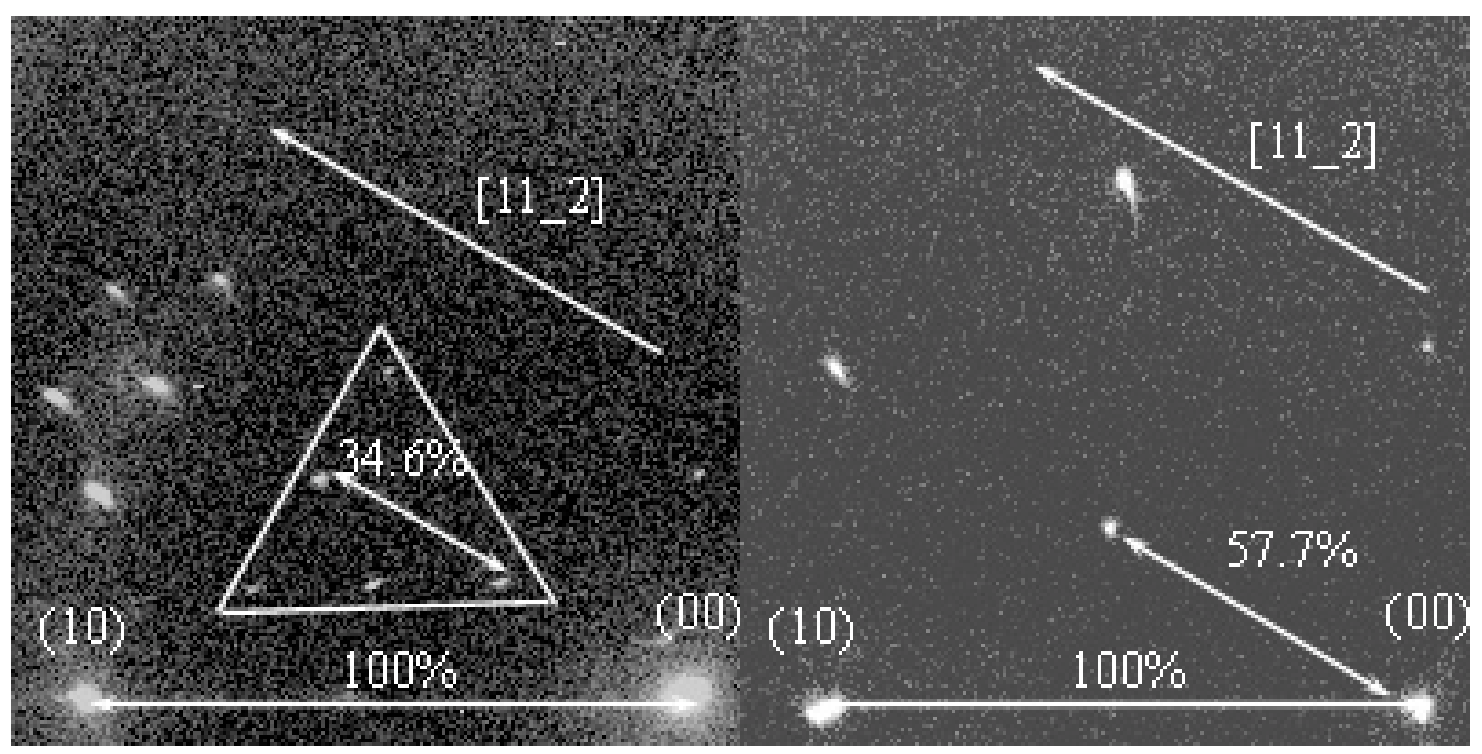

Figure 2 diffraction patterns of $P b \sqrt{ } 3 x \sqrt{ } 3$ (beta)phase and $\sqrt{3} x \sqrt{3}$ phase. The size of the images are $120 \% \times 120 \%$ BZ

The diffraction patterns of the two images are displayed in Fig.2. The beta phase's unit cell is $\sqrt{ } 3$ times of Si unit cell, so in reciprocal space, it is unit cell is $1 / \sqrt{ } 3$ of Si. There fore the position of beta spot is $1 / \sqrt{3}$ of $100 \%$, or $57.7 \%$ from the (00) spot. The $\sqrt{ } 7 x \sqrt{3}$ phase's unit vectors in reciprocal space can be calculated using formula:

$\vec{a}_{1}^{*}=2 \pi \frac{\hat{n} \times \vec{a}_{1}}{\vec{a}_{1} \bullet\left(\vec{a}_{2} \times \hat{n}\right)}, \vec{a}_{2}^{*}=2 \pi \frac{\vec{a}_{2} \times \hat{n}}{\vec{a}_{1} \bullet\left(\vec{a}_{2} \times \hat{n}\right)}$, where

$\hat{n}$ is the unit vector perpendicular to the paper plan, $a_{1}=\sqrt{ } 3 a_{0}, a_{2}=\sqrt{ } 7 a_{0}$ are the unit vectors in real space. Because the area of the unit cell $S=(5 \sqrt{ } 3 / 2) a_{0}{ }^{2}$ and we know that $S=a_{1} a_{2} \sin \theta$, we can get the angle $\theta$ between $a_{1}, a_{2}: \sin \theta=S /\left(a_{1} a_{2}\right)=5 / 2 \sqrt{ } 7$ therefore $\theta=\sin ^{-1}(5 / 2 \sqrt{ } 7)$.

Now we can calculate the unit vectors in reciprocal space:

$$
\begin{aligned}
& \vec{a}_{1}^{*}=2 \pi \frac{\hat{n} \times \vec{a}_{1}}{\vec{a}_{1} \bullet\left(\vec{a}_{2} \times \hat{n}\right)}=2 \pi \frac{a_{1}}{a_{1} a_{2} \sin \theta} \hat{a}_{1}^{*}=\frac{2 \pi}{a_{2} \sin \theta} \hat{a}_{1}^{*}=\frac{4 \pi}{5 a_{0}} \hat{a}_{1}{ }^{*}, \\
& \vec{a}_{2}^{*}=2 \pi \frac{a_{2} \times \hat{n}}{a_{1} \bullet\left(a_{2} \times \hat{n}\right)}=2 \pi \frac{a_{2}}{a_{1} a_{2} \sin \theta} \hat{a}_{2}^{*}=\frac{2 \pi}{a_{1} \sin \theta} \hat{a}_{2}^{*}=\frac{4 \pi \sqrt{7}}{5 \sqrt{3} a_{0}} \hat{a}_{2}^{*}
\end{aligned}
$$

Notice $100 \%$ Si Brillouin Zone(BZ) corresponds to $2 \pi \frac{\hat{n} \times \vec{a}_{01}}{\vec{a}_{01} \bullet\left(\vec{a}_{02} \times \hat{n}\right)}=\frac{4 \pi}{\sqrt{3} a_{0}} \hat{a}_{01}{ }^{*},\left(\vec{a}_{01}\right.$ and $\vec{a}_{02}$ are unit vector of Si unit cell, they have same magnitude $\mathrm{a}_{0}$ and the angle between them is $60^{\circ}$ ), the magnitude of the two unit vectors are 
$a_{1}^{*}=\frac{4 \pi}{5 a_{0}}=\frac{\sqrt{3}}{5} * 100 \% B Z=34.6 \% B Z$,
$a_{2}^{*}=\frac{4 \pi \sqrt{7}}{5 \sqrt{3} a_{0}}=\frac{\sqrt{7}}{5} * 100 \% B Z=52.9 \% B Z$

$\vec{a}_{1}^{*}$ is in [11_2] direction so in [11_2] direction, the positions of $\sqrt{ } 7 \mathrm{x} \sqrt{3}$ spots are $34.6 \%$, $69.2 \% \ldots$

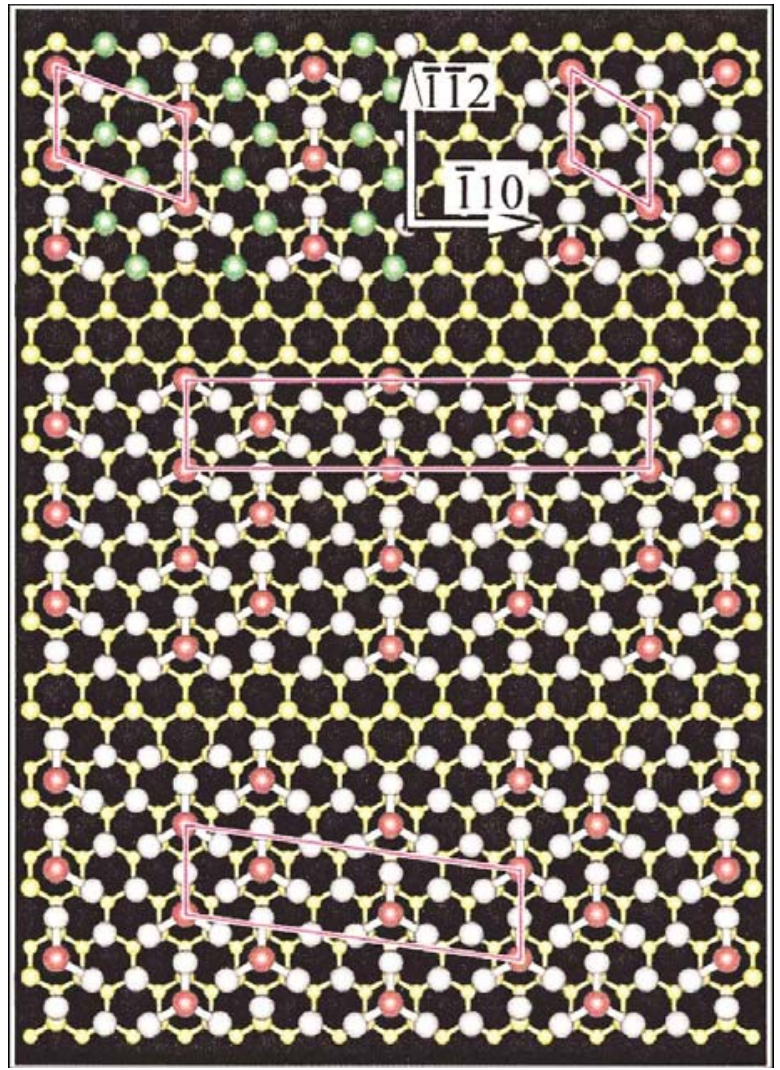

Figure 3 Schematic model of the $\mathrm{Si}(111)$ with several unit cells of the linear phases. The red atoms are $\mathrm{Pb}$ atoms. On the top is the $\sqrt{3} \times \sqrt{3}$ and $\sqrt{7} \times \sqrt{3}$ unit cells. In the middle gives the unit cell of $(3,1)$ devil's staircase and in the bottom is the unit cell of $(2,1)$ devil's staircase. (taken from [2])

When we make depositions on $\mathrm{Pb}$ beta phase at $\mathrm{T} \sim 180 \mathrm{~K}$, the beta phase will convert to $\sqrt{7} x \sqrt{3}$ phase and then $\sqrt{3} x \sqrt{3}$-alpha phase. The $\sqrt{3} x \sqrt{3}$-alpha phase has same unit cell as the $\sqrt{3} x \sqrt{3}$-beta phase, but there are 4 atoms in each cell, so its coverage is $4 / 3=1.33 \mathrm{ML}$. When the coverage is between 1.2ML and 1.33ML, the surface is the mixture of $\sqrt{ } 7 \mathrm{x} \sqrt{3}$ phase and $\sqrt{3} x \sqrt{3}$-alpha phase. It is known as Devil's staircase (DS). Fig.3 shows the schematic models for $\mathrm{DS}$ phases. The red dots are $\mathrm{Pb}$ atoms and the white dots are $\mathrm{Si}$ atoms. The $\mathrm{DS}$ phases are one dimensional phase and its unit cell is 


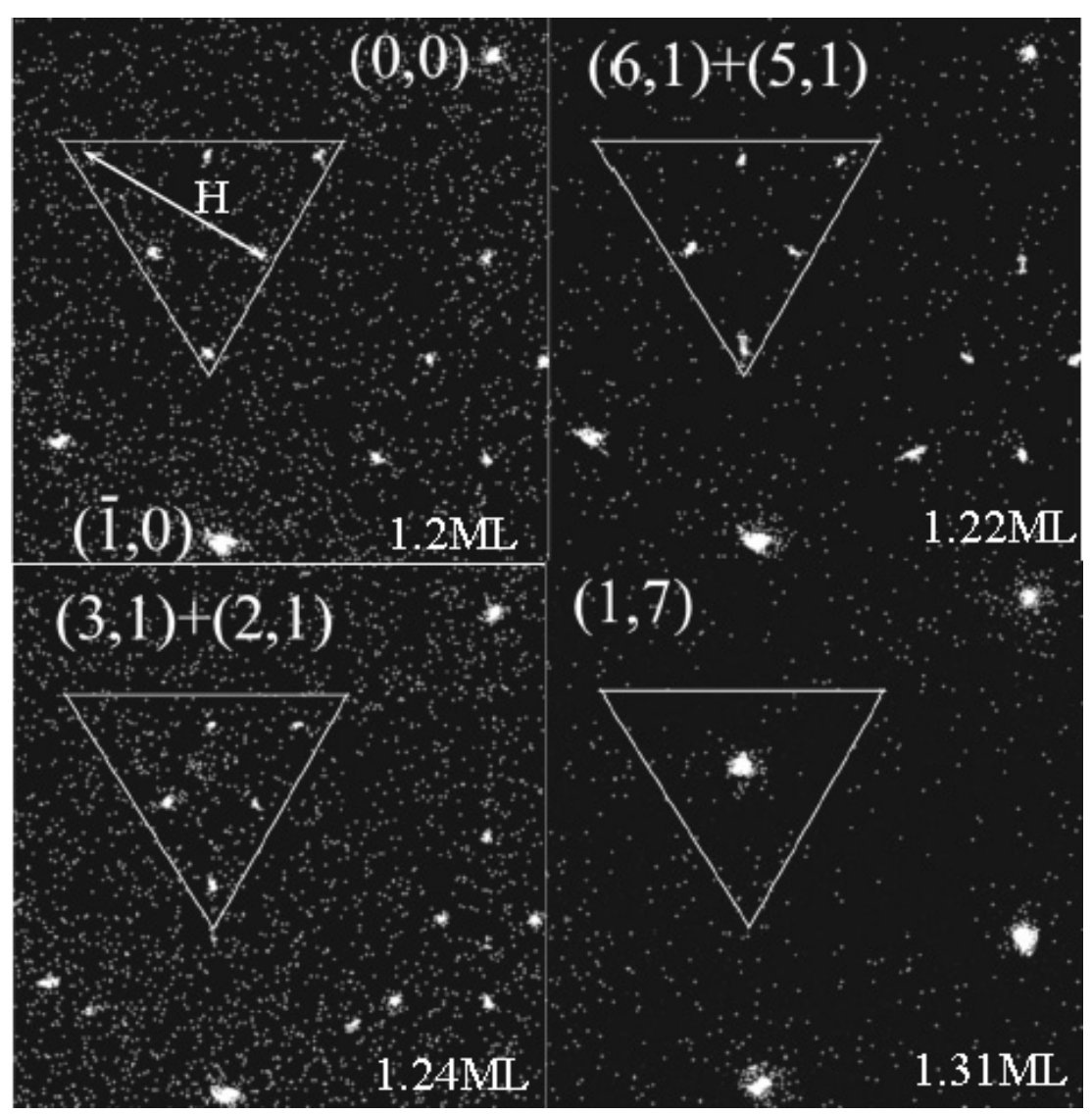

Figure 4 Stepwise deposition experiments at constant temperature $T=169 \mathrm{~K}$. The triangle formed by the spots decrease with coverage (marked in figures). (Taken from [3] combined by $n \sqrt{7} x \sqrt{3}$ cells and $m \sqrt{3} x \sqrt{3}$ cells. Each DS phase have different structure depends on the value of $(n, m)$ or the coverage. For a given phase $(n, m)$, the unit cell's length is $n *\left(5 a_{0} / 2\right)+m *\left(3 a_{0} / 2\right)=(5 n+3 m) a_{0} / 2$ and its height is $\sqrt{3} a_{0}$, so its area is $\sqrt{ } 3(5 n+3 m) a_{0}^{2} / 2$, or $(5 n+3 m) *($ area of Si unit cell). Each unit cell contains $6 n+4 m$ Pb atoms, so its coverage is $(6 n+4 m) /(5 n+3 m)$. For each coverage $\theta$, there is a pair of integer $(n, m)$ that satisfies $\theta=(6 n+4 m) /(5 n+3 m)$. For an example, for coverage $=1.25 \mathrm{ML}, \mathrm{n}=1, \mathrm{~m}=1$, and the unit cell of devil's staircase is made by one $\sqrt{ } 7 x \sqrt{ } 3$ cell and one $\sqrt{ } 7 x \sqrt{3}$ cell.

$\operatorname{An}(n, m)$ DS phase in $\mathrm{Pb} / \mathrm{Si}(111)$ has unit cell vectors $b_{2}=\sqrt{ } 3 a_{0} \hat{y}$, $b_{1}=\sqrt{3} a_{0} / 2 \hat{y}+\left[(m+n) a_{0} / 2\right] \hat{x}$ [if $m+n$ is odd $]$ and $b_{1}=\left[(m+n) a_{0} / 2\right] \hat{x}$ [if $m+n$ is even] with period $q=(5 n+3 m) \mathrm{a}_{0} / 2$. The diffraction pattern resulting from such a phase also has three-fold symmetry. Fig.4 shows diffraction patterns for some DS phase. The first one is just $\sqrt{ } 7 \mathrm{x} \sqrt{3}$ phase (1.2ML), the second one is mixture of $(6,1)$ and $(5,1)(1.22 \mathrm{ML})$, the third one is mixture of $(3,1)$ and $(2,1)(1.24 \mathrm{ML})$, and the last one is $(1,7)(1.31 \mathrm{ML})$. The diffraction 
patterns of DS phases are similar to the $\sqrt{ } 7 x \sqrt{3}$ phase, but the size of the triangle (can be characterized by the parameter $H$ ) is dependent on the coverage. It decreases when $\sqrt{3} \sqrt{3}$ components increase and when coverage increases to $1.33 \mathrm{ML}$ it will shrink into a single point at $57.7 \%(1 / 3,1 / 3)$.

The intensity of diffraction pattern can be expressed as the product of two terms, i.e., the scattering factor within a single $(n, m)$ unit cell $F_{\text {unit cell }}$ times the reciprocal lattice of Dirac $\delta$-functions originating from long-range order

$$
I_{\text {total }}\left(k_{x}, k_{y}\right)=\left|F_{\text {unitcell }} \sum_{l, s}^{s=n+m} \exp \left(i k_{x} \cdot l b_{1}+k_{y} s b_{2}\right)\right|^{2}
$$

Next we will show the relation between $H$ and the phase period for the $(n, 1)$ phases, which are observed in the calibration. The $\delta$-functions are separated in the [11_2] direction by $\delta=4 \pi / a_{0}(5 n+3)=173.2 \% /(5 n+3)$. The spots are expected at multiple of $\delta$. For the $(\mathrm{n}, 1)$ phases, the $\sqrt{7} \mathrm{x} \sqrt{3}$ phase is dominant, and $F_{\text {unitcell }}$ is strong near the $\sqrt{7} \mathrm{x} \sqrt{3}$ spots. Therefore the strongest $\delta$-function spots are the two intersections at the triangle vertex and the spot and at the middle of the opposite side. The distance between them is the triangle height $H$. Since these spots are separated by a multiple of the $\delta$-function spacing and are the closest spots to the $1 / 5$ and $2 / 5$ locations of the $173 \% \mathrm{BZ}$ diagonal, $H$ is the largest possible integer multiple of the $\delta$ - function separation which is less than $1 / 5$, the multiple is

$$
H / \delta=\operatorname{int}\left(\left(4 \pi / 5 \mathrm{a}_{0}\right) /\left(4 \pi / \mathrm{a}_{0}(5 n+3)\right)=\operatorname{int}((5 n+3) / 5)=n\right.
$$

So $H=n \delta=173.2 \% * n /(5 n+3)$. Therefore, if we take 1D scans in [11_2] direction, by measuring $H$, we can calculate the coverage of DS. Fig.4 gives the $2 \mathrm{D}$ diffraction patterns at different coverages which show the shrinking of the triangle.

In a word, when we make depositions on $\mathrm{Pb}$ beta phase, we will see the beta spots become weaker and the $\sqrt{7} x \sqrt{3}$ spots become stronger. When the coverage exceeds 1.2ML, the position of $\sqrt{ } 7 x \sqrt{3}$ spots will move due to we have entered DS phases(as seen in fig.4, and an example will be given in next paragraph). Therefore we can first make a good $\sqrt{3} x \sqrt{3}$-beta phase, and then make small depositions until we reach the $\sqrt{ } 7 x \sqrt{ } 3$ phase or DS. The total amount of deposited $\mathrm{Pb}$ would be then the difference of the final phase and initial phase, and we can divide it by the deposition time and get the flux rate. The key of this calibration is to 
make a good initial beta phase and find the correct final coverage. The beta phase is made by depositing $\mathrm{Pb}$ to slightly above $1 / 3 \mathrm{ML}$ (the exact amount is not important) and annealing the sample to make the $\mathrm{Pb}$ atoms evaporate. $\mathrm{We}$

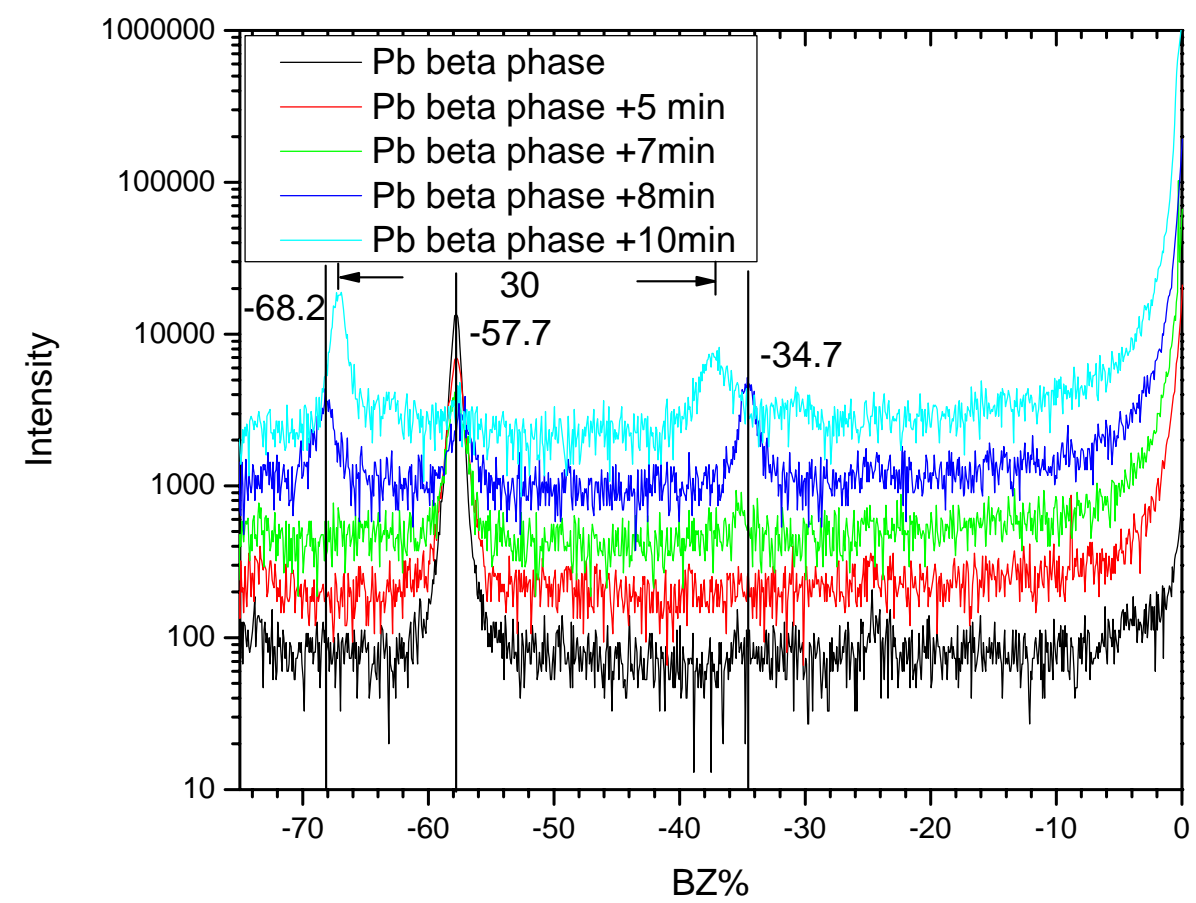

Figure 12 Calibration for $\mathrm{Pb}$ source flux rate by making depostions on $\mathrm{Pb}$ beta phase at $\mathrm{T}=170 \mathrm{~K}$. The profiles are taken along [11_2] direction. The $57.7 \%(\sqrt{3} / 3)$ spot is from beta phase and the $-68.2 \%(2 \sqrt{3} / 5)$ spot is from $\sqrt{7} \times \sqrt{3}$ phase. The small shift of the spot's postion in the last curve indicates the coverage is already slightly exceeds 1.2ML.

take 1-D scans to keep measuring the intensity of the beta spot. Then its intensity reaches the maximum, the surface is covered by beta phase completely and the coverage at this time is $1 / 3 \mathrm{ML}$. Then we cool the system down for further depositions. $\mathrm{Pb}$ is added in small amounts ( $\sim 0.1 \mathrm{ML}$ at a time). The beta spots disappear and the $\sqrt{7} \mathrm{x} \sqrt{3}$ spots begin to appear gradually with depositions. When the beta spots completely disappear and the $\sqrt{7} x \sqrt{ } 3$ phase is reached, the surface coverage can be determined.

This is an example for $\mathrm{Pb}$ source calibration. The source is heated by applying a current of 2.6A. Several depositions are made on $\mathrm{Pb}$ beta phase, after each deposition the sample is annealed to above room temperature and then cooled down to $170 \mathrm{~K}$ and take scans. 
The first dose is 5 minutes, then 2 minutes, 1 minute, 2 minutes. Fig. 5 shows the 1-D scans along [11_2] direction after each deposition. We can tell from the figure that beta spot intensity decrease from 13000 after each dose to 300 (which is the level of back ground noise). After the third dose and the $\sqrt{7} x \sqrt{3}$ spots appears (intensity 547) after the third dose. After the fourth dose, the position of $\sqrt{ } 7 x \sqrt{3}$ spots (intensity 1173 ) moves (the $-68.2 \%$ spot to the right and the $-34.8 \%$ spot to the left), which means we are in DS phase already so we stop further deposition.

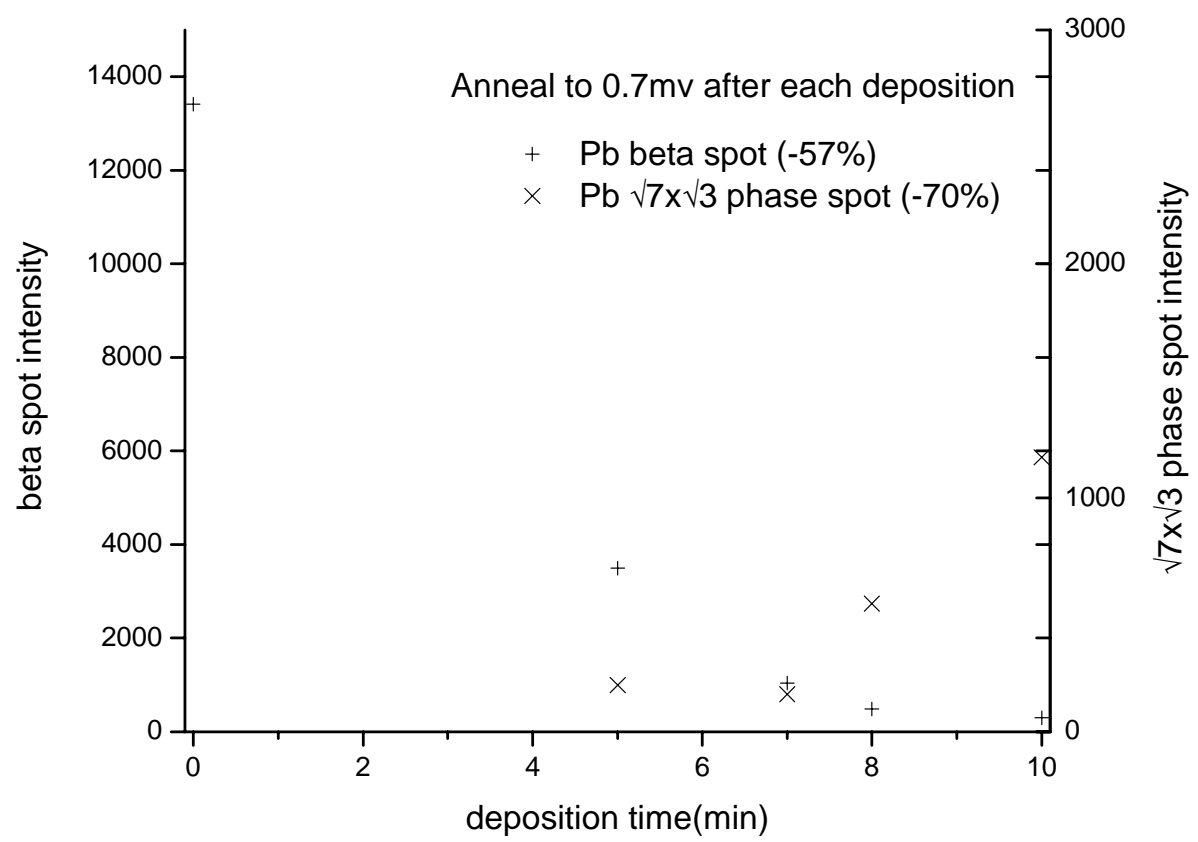

Figure 6 The intensity of beta spot and $\sqrt{7} \mathbf{x} \sqrt{3}$ phase spot dependence on deposition time.

Fig. 6 gives the intensity of beta spot and $\sqrt{ } 7 x \sqrt{3}$ phase spot dependence on deposition time. The intensity of spots are measured from the curves taken from Fig.5. It shows clearly the transition from beta phase to the $\sqrt{ } 7 x \sqrt{ } 3$ phase: the intensity of beta spot goes to zero and the intensity of In $\sqrt{ } 7 x \sqrt{ } 3$ spot arise from zero.

In the last curve, we already have DS phase. Since $H=173.2 \% * \mathrm{n} /(5 \mathrm{n}+3)$, and in the last 1-D file (10 minute deposition) in Fig.3 we find $H=30 \%$, so $\mathrm{n}=4$ and the coverage is $(1.2 \mathrm{n}+1.33) /(\mathrm{n}+1)=1.23$. Therefore the flux rate of $\mathrm{Pb}$ source is $(1.23-0.33) / 10=0.09 \mathrm{ML} / \mathrm{min}$.

After calibration of $\mathrm{Pb}$ source, we can use a quartz crystal microbalance (QCM) to determine the amount of material deposited on the surface. A QCM contains a small quartz 
crystal near the path from the source to the sample, but not exactly between them, avoiding blocking the atom stream during depositions. The crystal is oscillating and its resonant frequency is proportional to one over the square root of its own mass:

$$
f=\frac{1}{2 \pi} \sqrt{\frac{k}{m}}
$$

Where $\mathrm{f}$ is the frequency, $\mathrm{m}$ is the mass of the quartz, $\mathrm{k}$ is Hook's constant. When we performing deposition experiments, the atoms beam will hit the quartz and therefore its mass will accumulate. As a result, the oscillating frequency will decrease. Because the change of the quartz mass can be considered as infinitesimal, the change of quartz frequency is linearly dependent on the change of the quartz' mass:

$$
\frac{d f}{d m}=\frac{1}{2 \pi}\left(-\frac{1}{2 m}\right) \sqrt{\frac{k}{m}}=-\frac{f}{2 m}
$$

During a typical depositing experiment, the change of $f$ and $m$ are less than $0.01 \%$ (the reading of the frequency is in the order of $\mathrm{MHz}$ (In the past 3years, the reading decreased from 3.3 MHz to $3.2 \mathrm{MHz}$ ), while the frequency change after $1 \mathrm{ML}$ of In deposition is only in the order of $100 \mathrm{~Hz}$ or less than $0.01 \%$ of its magnitude, so they can be considered as constant. By measuring the change of frequency before deposition and after, one can determine the mass change of the quartz crystal, and therefore the coverage. As long as we learn the frequency change with a known amount of material deposition, then the coverage can be expressed in terms of the change of oscillation frequency.

For In source deposited at $\mathrm{T}=185 \mathrm{~K}, 1 \mathrm{ML}$ corresponds to $80 \mathrm{~Hz}$ of frequency difference. This parameter is determined by measuring the calibrated $\mathrm{Pb}$ source. Since the $\mathrm{Pb}$ source flux is calibrated using the method in previous part, we find the frequency difference $\Delta \mathrm{f}=150 \mathrm{~Hz}$ when we deposit 10 minutes of $\mathrm{Pb}$ at flux rate $0.1 \mathrm{ML} / \mathrm{minute}$. Because In atom's mass(115) is less than $\mathrm{Pb}(207)$, the mass of $1 \mathrm{ML}$ of $\mathrm{In}$ is $115 / 207=55.5 \%$ of $1 \mathrm{ML}$ of $\mathrm{Pb}$, so $1 \mathrm{ML}$ of In corresponds to $\Delta \mathrm{f}=150 * 55.5 \%=80 \mathrm{~Hz}$.

The following table gives an example of In source calibration using the quartz. A current of $2.6 \mathrm{~A}$ is applied on the source and the temperature is $185 \mathrm{~K}$. We measure the reading of quartz after each deposition. Using this table, we can make a frequency-deposition plot and make a linear fit in figure 7 . The slope of the linear fitting is $-7.85 \mathrm{~Hz} / \mathrm{min}$. So the 
flux rate is $7.85 / 80=0.1 \mathrm{ML} /$ minute. The sensitivity of the QCM provides Hz-level sensitivity in frequency so we can have submonolayer accuracy in the coverage measurements.

\begin{tabular}{lrr}
$\begin{array}{l}\text { Deposition time } \\
\text { (minute) }\end{array}$ & \multicolumn{2}{l}{$\begin{array}{l}\text { Frequency } \\
(\mathrm{Hz})\end{array}$} \\
& 0 & 4854650 \\
3 & 4854612 \\
4 & 4854608 \\
6 & 4854589 \\
10 & 4854561 \\
13 & 4854537 \\
16 & 4854521
\end{tabular}

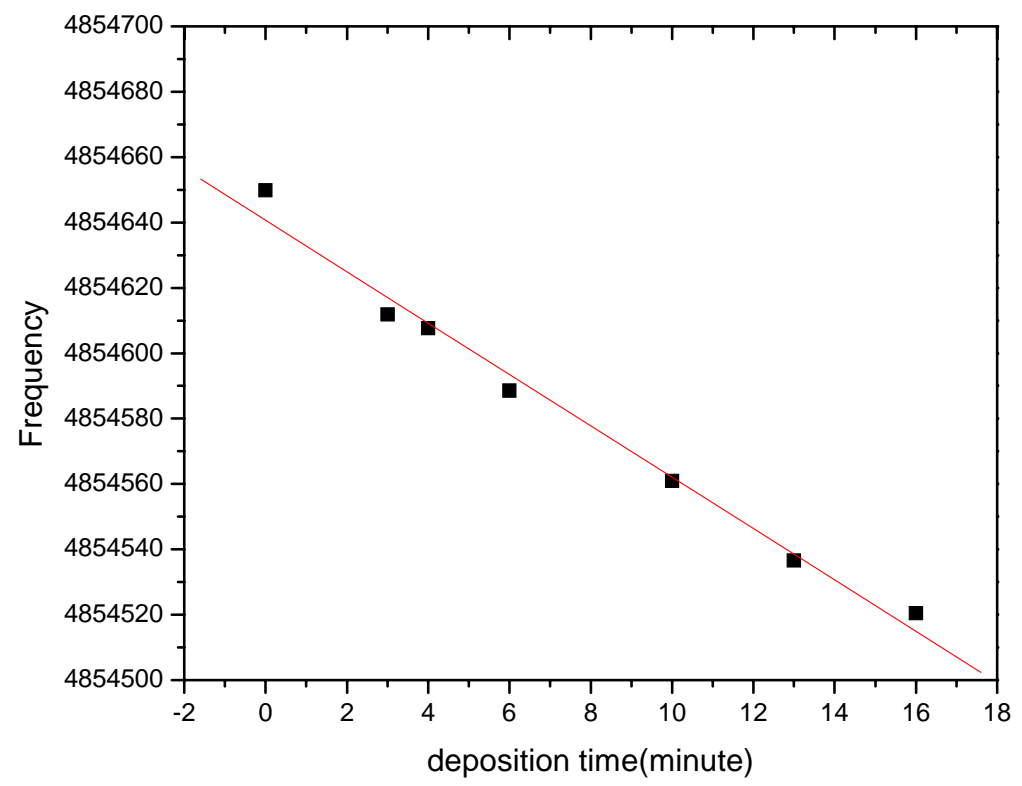

Figure 7 The frequency of the quartz and the deposititon time when In are deposited at $T=185 \mathrm{~K}$. From the slope of the linear fit we can determine the flux rate of In. 
The advantage of using a QCM to determine the amount of deposition is that as long as we have the proportional constant $\mathrm{df} / \mathrm{dm}$ (or the frequency difference per ML), we don't need to calibrate the flux rate any more when we change the flux rate by changing the temperature of the source since the frequency change is only dependent on the total amount, not flux rate. When read the frequency of the QCM, it is important that the two reading must be get at the same temperature because the frequency reading is dependent to the temperature, too.

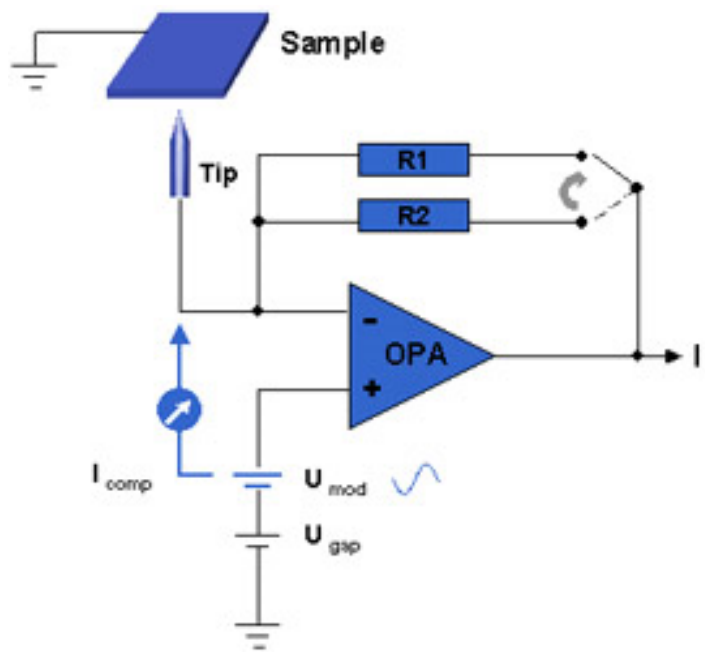

Figure 8 Schematic diagram of the STM (figure taken from http://www.omicroninstruments.com/ltstm/STM.html)

One of the main instruments we use is Scanning tunneling microscope (STM). Fig. $8^{[4]}$ gives a schematic drawing showing how STM works. We make a very sharp tip and approach it near the surface of a sample. When we apply a voltage between the tip and the sample, there would be a tunneling current between them. When the tip moves around the sample, due to the unsmooth of the sample surface, the distance between the sample and the tip will vary, and the tunneling current is exponentially dependent on the distance, which make the tunneling current very sensitive to the surface condition of the sample. After we survey a certain area of a sample, we can get a good surface profile of the sample. In real experiments, it's the current, not voltage kept constant. The tip is controlled by electronic system via a piezoelectric crystal, and when the tip moves around the sample, there is a feed back system controlled by electronic instruments to keep the height of tip to maintain the tunneling current constant. 
STM gives the best ever real space resolution in all existing instruments, and this is its biggest advantage. Currently only STM can get images with atomic resolution. What's more, it provides a visual surface profile in real space which can be easily understood by anybody.

The tip in STM is controlled by the piezoelectric crystal, so it is essential to calibrate the piezoelectric crystal to get the correct reading. Because we can get atomic resolution on a properly prepared good $\mathrm{Si}(111) 7 \times 7$ surface and the unit cell of $\mathrm{Si}$ is well known, it is convenient to calibrate in the lateral direction of the surface. To calibrate in the vertical direction (i.e. the direction perpendicular to the (111) plane), we can move the tip to a terrace with steps. By measuring the steps height in STM and compare with well known value the vertical direction can be correctly calibrated. Fig.9 gives an STM image of clean Si surface with steps and its cross-section. It shows the step height is $0.435 \mathrm{~nm}$, but the actually height can be calculate from its fcc structure and lattice constant: $0.384 \mathrm{~nm} * \sqrt{ }(2 / 3)=0.31 \mathrm{~nm}$, therefore all the vertical heights measured in STM need to multiplied by a coefficient $0.31 / 0.435=0.72$ to get their real values. 


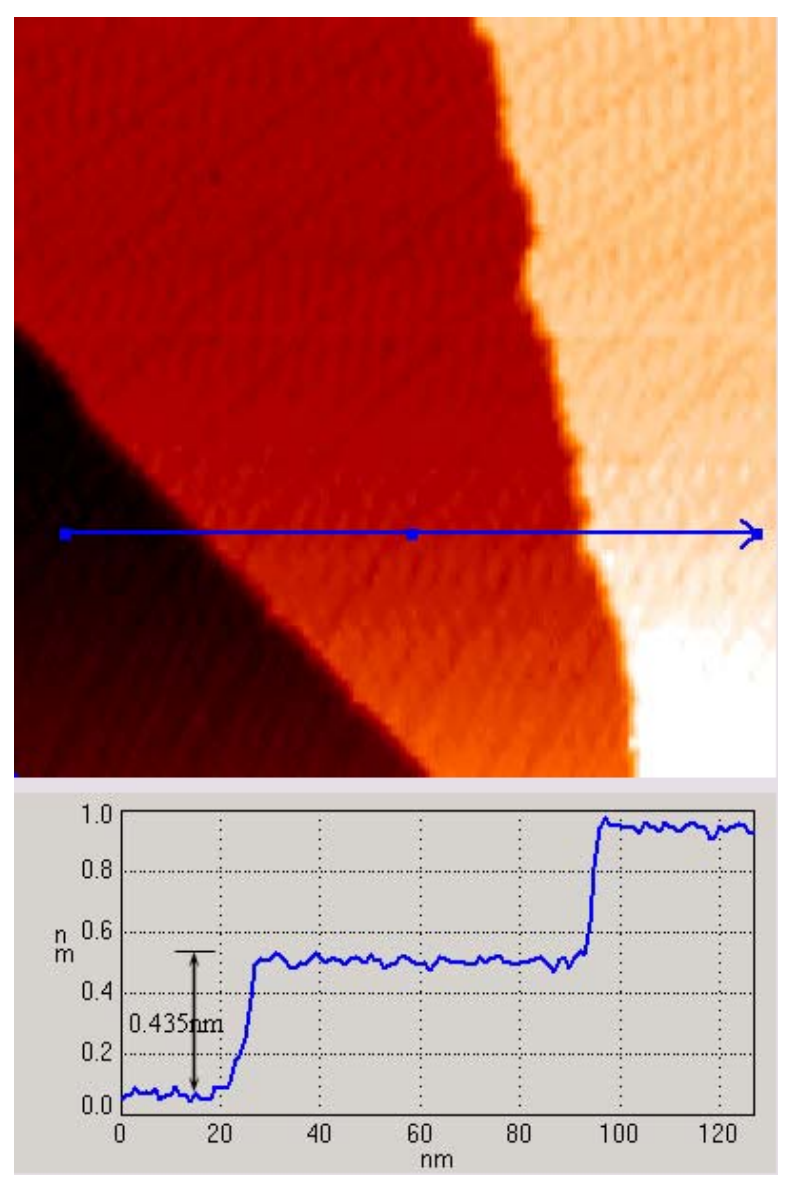

Figure 9 a 140nm x 140nm STM image for clean Si surface with steps. The 1-D cross-section shows that its step height is $0.435 \mathrm{~nm}$.

If we move the tip for a long distance or the temperature is changed (e.g. move the tip horizontally for $1 \mu \mathrm{m}$, which is 5-10 times more than the size of a typical STM image), the piezoelectric crystal will deform elastically in some degree and there will be distortion in the image. Elastically means the deformation is not permanent and it takes some time (usually a few minutes) to return to normal and stabilize the tip and get rid of the distortions to obtain reliable measurement.

The calibration of flux rate in STM experiments is done by calculating the total volume in a certain time on the surface. For an example, in the In on $\mathrm{Pb}$ alpha phase experiments, we take an STM image after each deposition at the same area.

The first thing to do after taking the images is to properly subtract background. Theoretically, the tip should move in a plane parallel to the surface, but in reality there is a small angle and the result is the STM images of the surface look like tilted. We need to eliminate that effects and correct the tilt. After that, we get the height of each point in the 
STM image. The collecting of STM images and background subtraction is done using a software called Scala. It controls the electric feedback system to keep the tunneling current constant and collect raw data. After collecting raw data it can also correct the background from tilting by interpolating in the points or areas with same altitude.

We have two methods to process the data. In the first method, we can divide the height axis into many small intervals $(0-0.01 \mathrm{~nm}, 0.01-0.02 \mathrm{~nm}$, etc, which can be considered as infinitesimals compared to the islands heights in order of $\mathrm{nm}$, for the images in fig. 10, the axis are divided into 500 intervals), and count there are how many pixels falling in each small intervals. Then we can draw a histogram that gives the relation between the number of pixels for each small height interval and the respect height(h). (see Fig.10) Because each pixel can be considered as an infinitesimal area, if we sum up all the counts over all the intervals we are practically performing integration over the axis and getting the total area of the image (in unit of pixels) and if we sum up the product of the count and its respective height (which is the volumes corresponding to pixels falling into each specific height interval) over all intervals we can get the total volume of the indium (in unit of pixel*nm). By dividing the total volume by the total area we can get the average height of In, i.e. the coverage of In deposited on the surface. After we get the coverages at different deposition times, the flux rate can then be obtained from the slope of the linear fit of the coverage-deposition time curve.
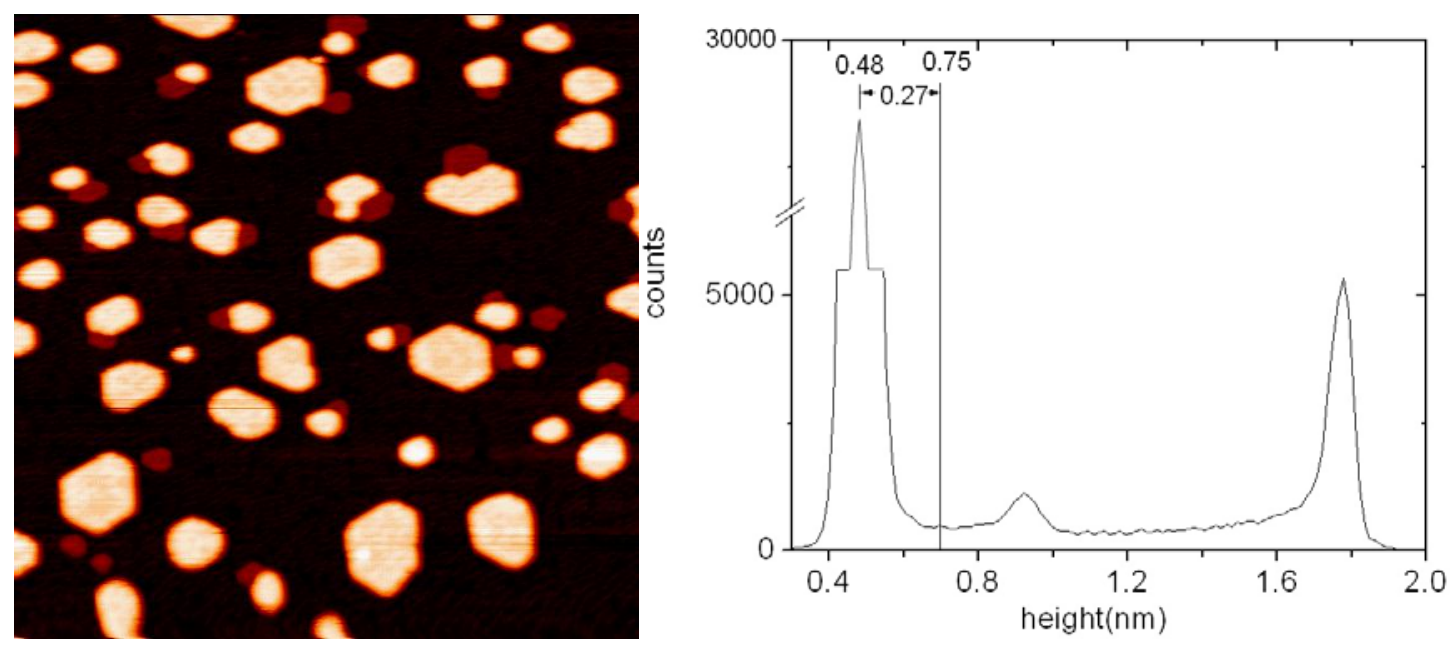

16minutes deposition. Total area(sum the counts in the whole axis) $=250000$, Total volume(sum of the product of count and height in the whole axis) $=187243$, and averaged height $=187243 / 250000=0.27 \mathrm{~nm}$. (0.62ML) 

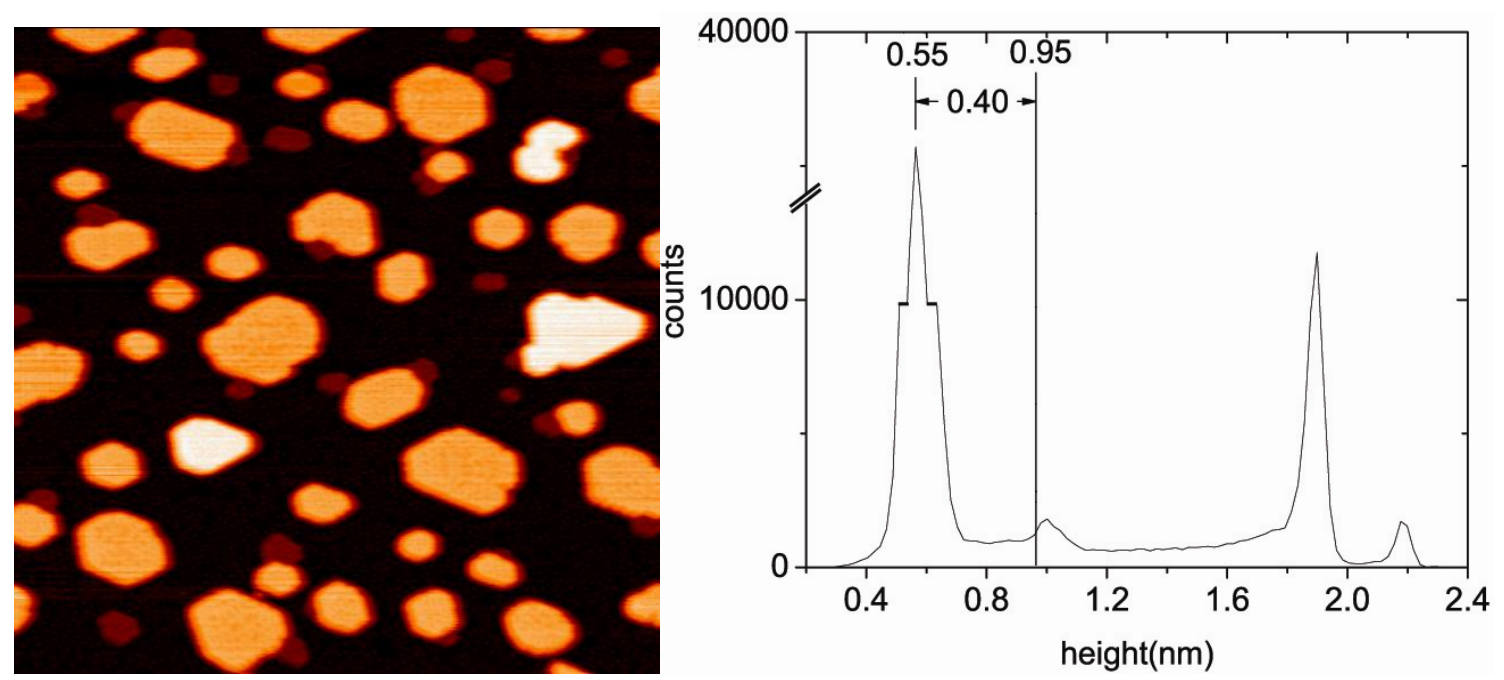

20minutes deposition. Total area $=237444$, Total volume $=250000$, and averaged height $=237444 / 250000=0.40 \mathrm{~nm} .(0.92 \mathrm{ML})$
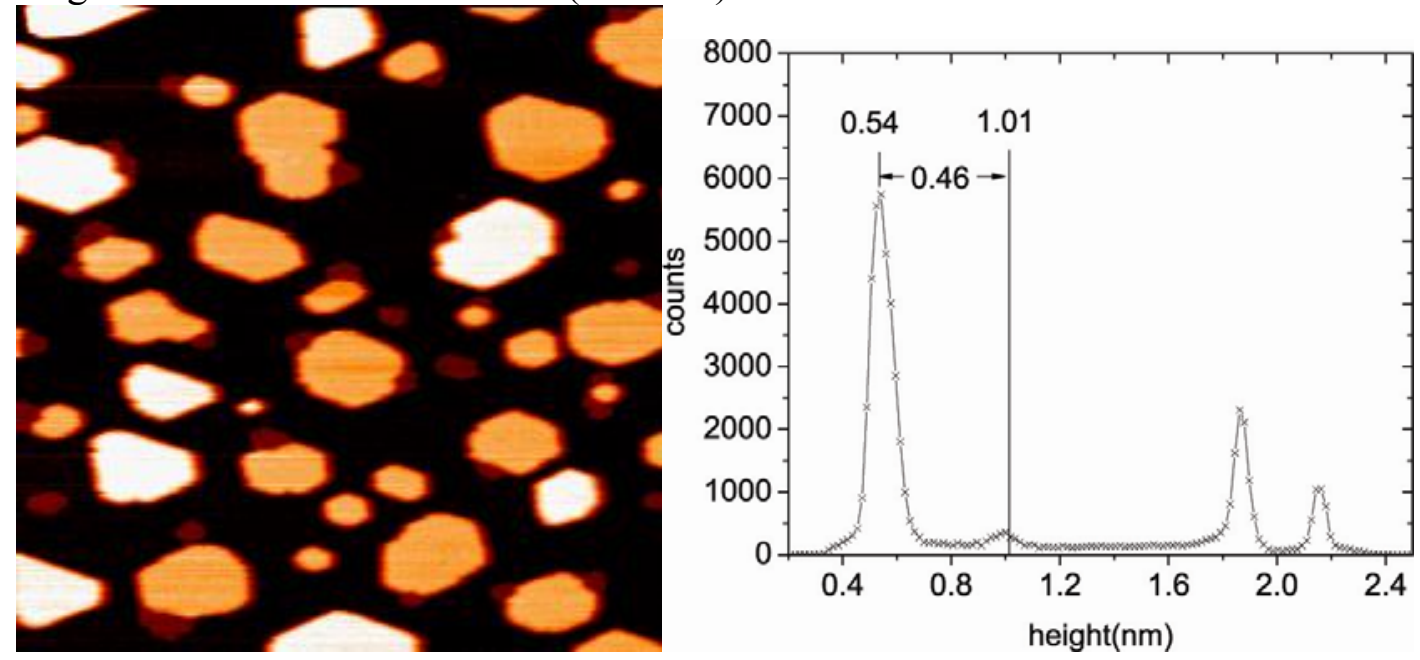

24minutes deposition. Total area $=62500$, Total volume $=62926$, and averaged height $=62926 / 62500=1.01 \mathrm{~nm}$. Coverage $=0.46 \mathrm{~nm}(1.06 \mathrm{ML})$
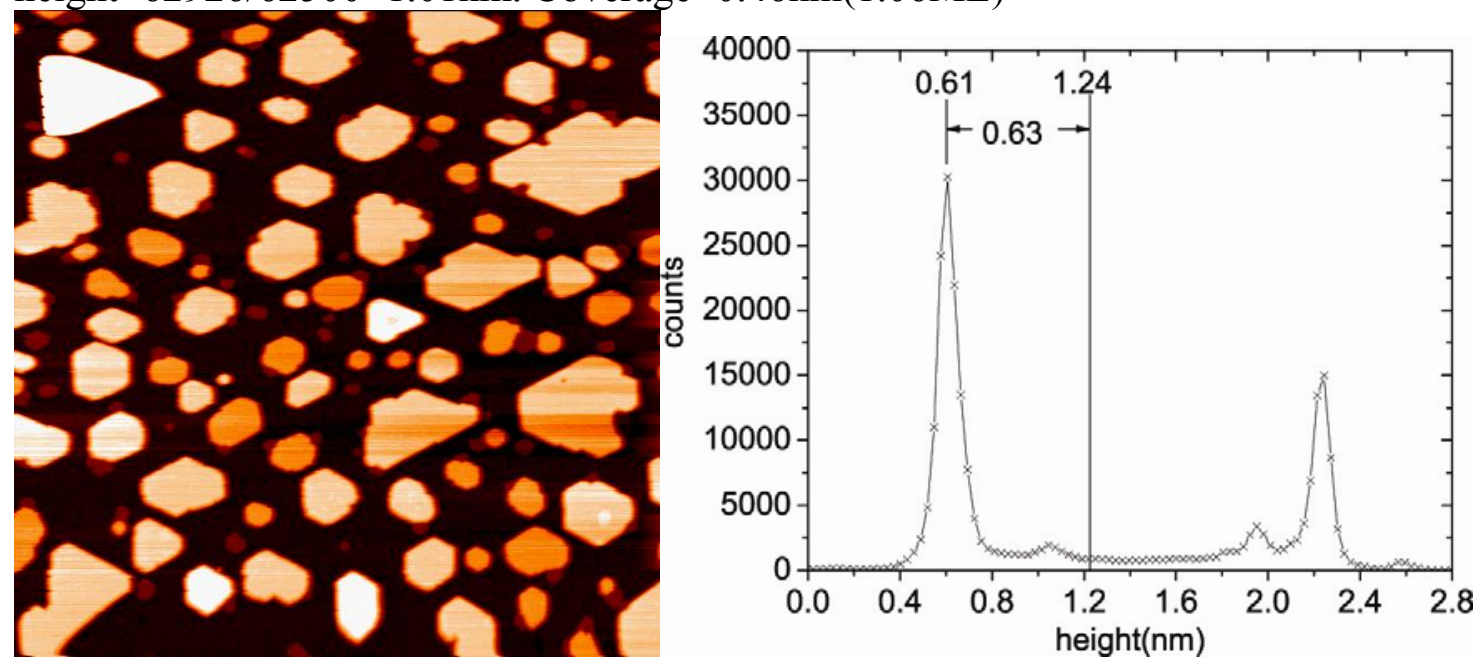
28 minutes deposition. Total area $=250000$, Total volume $=309013$, and averaged height $=309013 / 250000=1.24 \mathrm{~nm}$. Coverage $=0.63 \mathrm{~nm}(1.45 \mathrm{ML})$
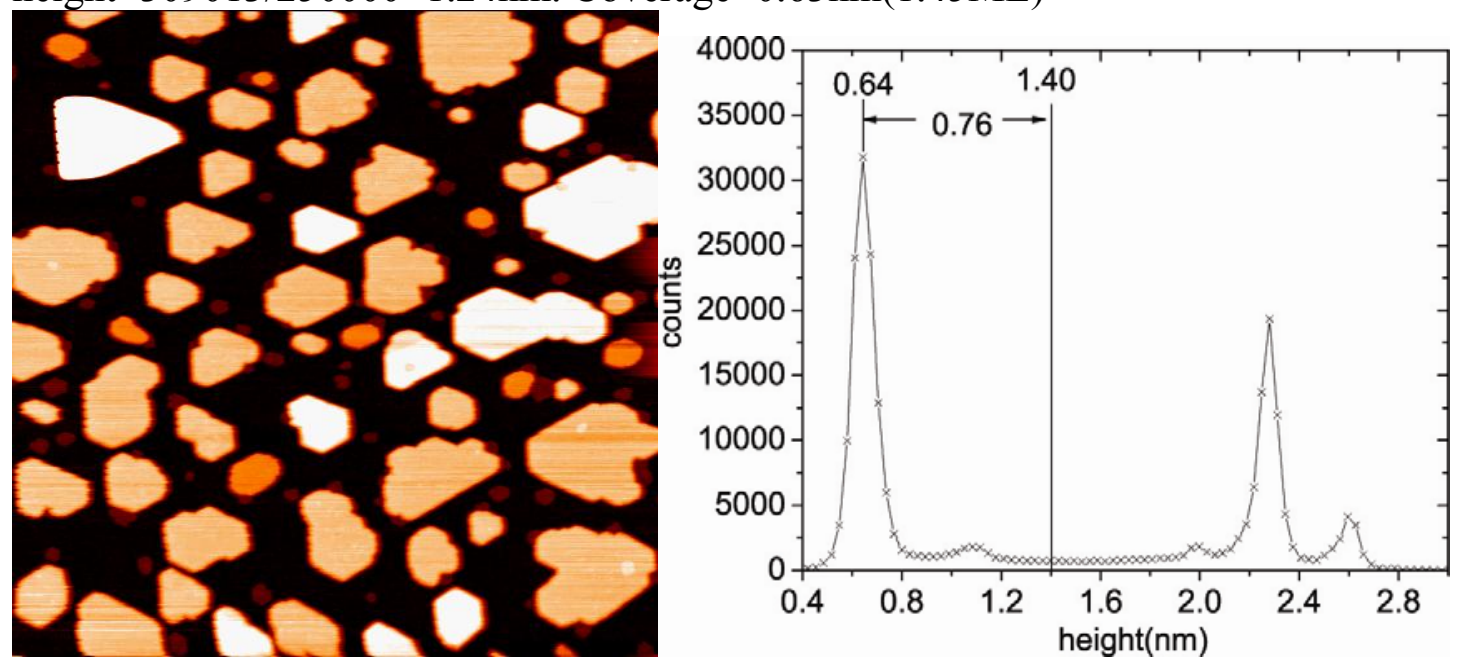

32 minutes deposition. Total area $=250000$, Total volume $=350335$, and averaged height $=350335 / 250000=1.40 \mathrm{~nm}$. Coverage $=0.76 \mathrm{~nm}(1.75 \mathrm{ML})$
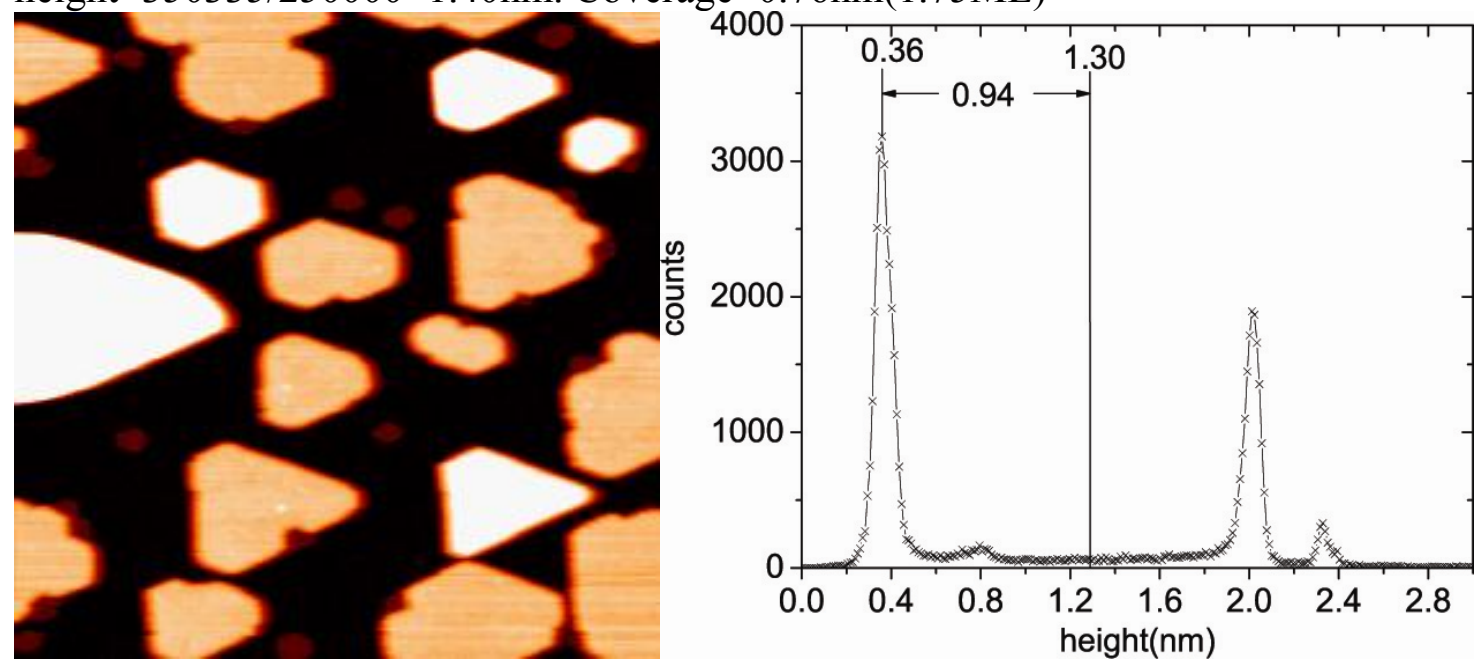

36 minutes deposition. Total area $=62500$, Total volume $=81092$, and averaged height $=81092 / 62500=1.30 \mathrm{~nm}$. Coverage $=0.94 \mathrm{~nm}(2.16 \mathrm{ML})$ 

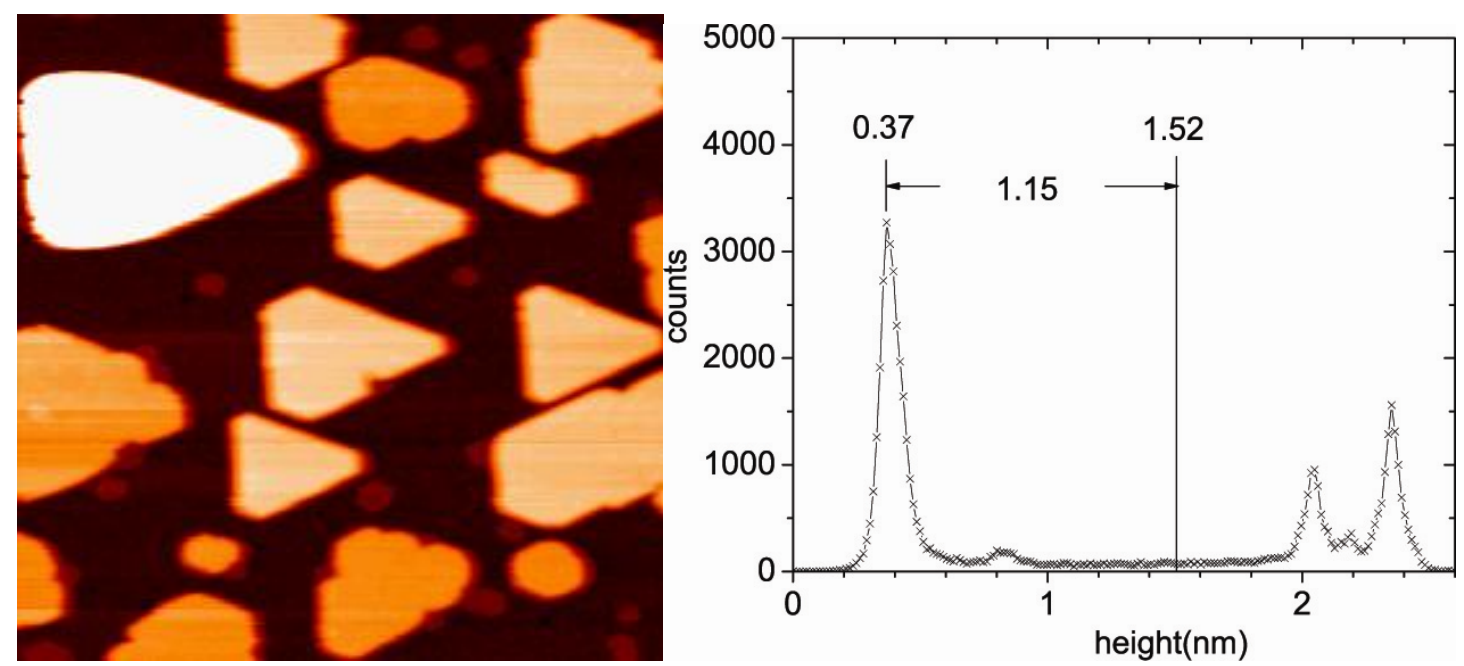

41 minutes deposition. Total area $=62500$, Total volume $=94853$, and averaged height $=94853 / 62500=1.52 \mathrm{~nm}$. Coverage $=1.15 \mathrm{~nm}(2.64 \mathrm{ML})$
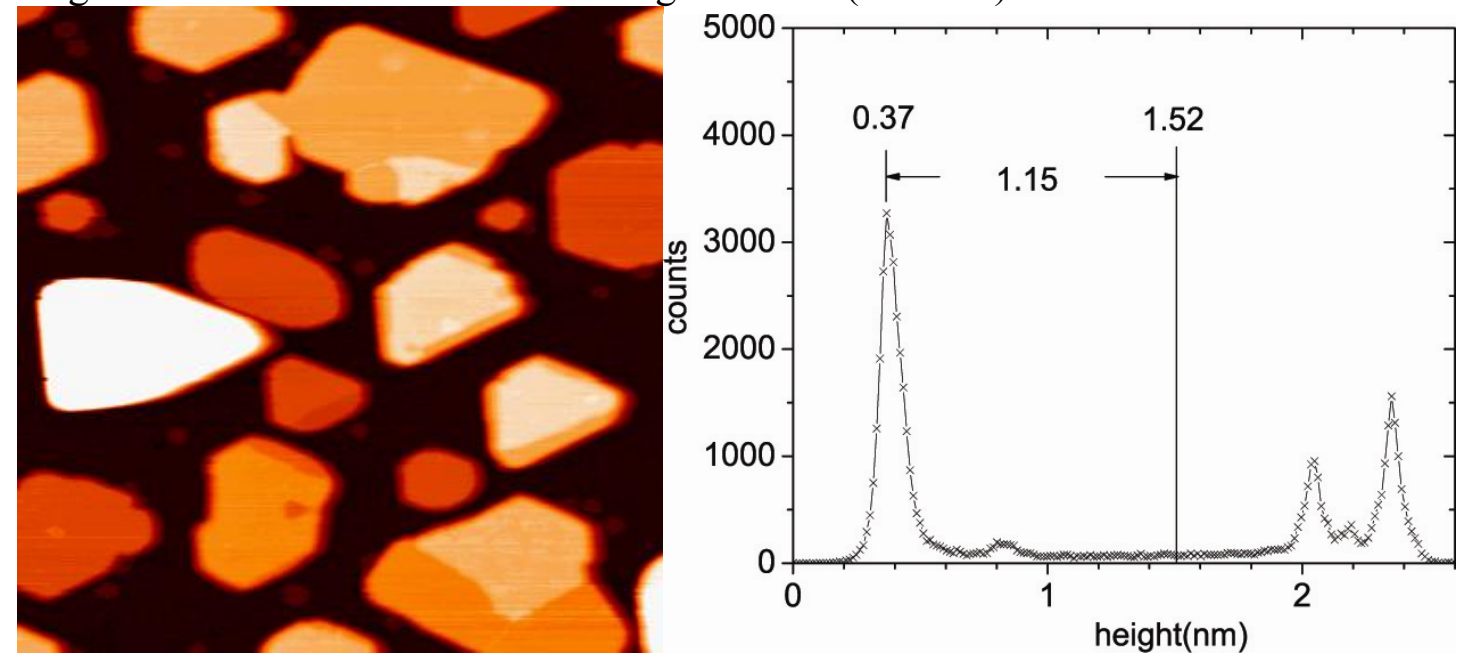

48minutes deposition. Total area $=122550$, Total volume $=308273$, and averaged height $=308273 / 122500=2.52 \mathrm{~nm}$. Coverage $=1.54 \mathrm{~nm}(3.54 \mathrm{ML})$

Figure 10 STM images and their histograms for In on Pb alpha phase. (16,20,24,28, 32,36,41,48, 56 minutes of In deposition, respectively)

For an example, Fig.10 shows a series STM images at different coverages and their histogram, respectively. In each histogram, we can calculate the coverage by summing up the data in the histogram to get the total area and total In volume. The average In height is just the total volume divided by the total area. The coverage (in unit of $\mathrm{nm}$ ) is the height from the wetting layer to the average height. Since the height of a single In layer is $0.38 \mathrm{~nm}$ in STM, it can also be converted to coverage in ML. The coverage is marked in each histogram based on the result of calculation. The analysis result of this images can be summarized as the following table: 


$\begin{array}{ccrrrr}\begin{array}{c}\text { Deposition } \\ \text { time(min) }\end{array} & \begin{array}{c}\text { Area } \\ \text { (pixel) }\end{array} & \begin{array}{c}\text { Volume } \\ (\text { pixel*nm) }\end{array} & \begin{array}{c}\text { average In } \\ \text { height } \\ (\mathrm{nm})\end{array} & \begin{array}{c}\text { wetting layer } \\ \text { height } \\ (\mathrm{nm})\end{array} & \begin{array}{c}\text { Coverage } \\ (\mathrm{nm})\end{array} \\ 16 & 250000 & 187243 & 0.75 & 0.48 & 0.27 \\ 20 & 250000 & 237444 & 0.95 & 0.55 & 0.40 \\ 24 & 62500 & 62926 & 1.01 & 0.54 & 0.46 \\ 28 & 250000 & 309013 & 1.24 & 0.61 & 0.63 \\ 32 & 250000 & 350335 & 1.40 & 0.64 & 0.76 \\ 36 & 62500 & 81092 & 1.30 & 0.36 & 0.94 \\ 41 & 62500 & 94853 & 1.52 & 0.37 & 1.15 \\ 48 & 122500 & 308273 & 2.52 & 0.98 & 1.54\end{array}$

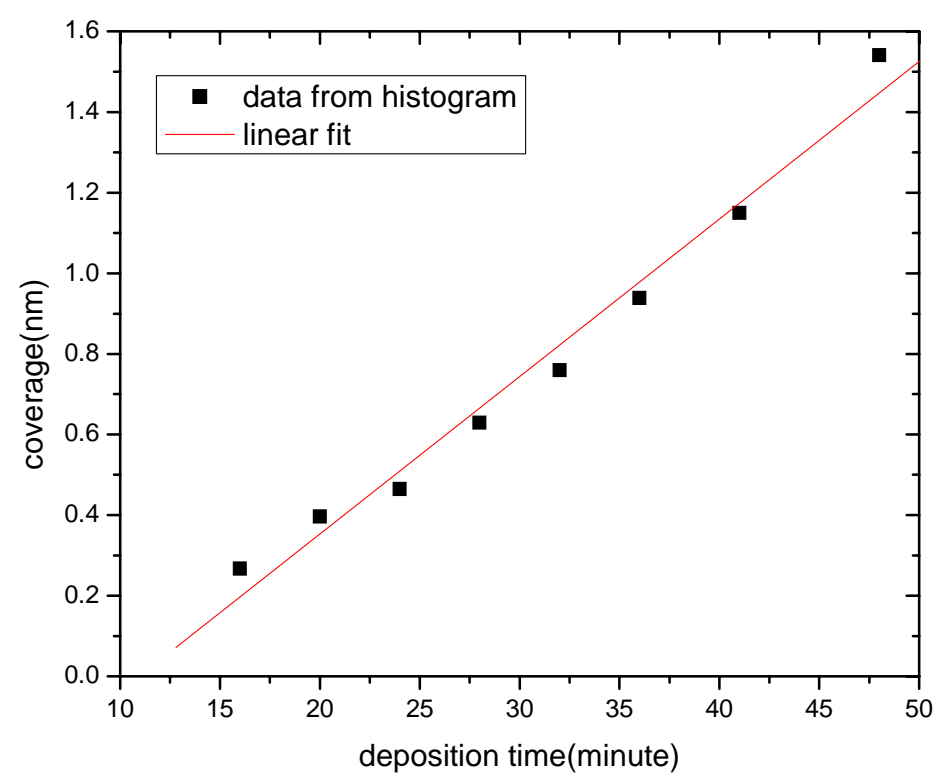

Figure 11 Linear fit of the coverage vs deposition time used to determine the flux rate of In depositions for STM. The dots are the coverages calculated from the histograms in respective time. We can calculate the flux rate from the slope of the fit.

Using the table, we can plot the time-coverage relation (fig.11) and get the linear fitting:

Coverage $=-0.428+$ deposition time $* 0.039$

This formula shows that the flux rate is $0.039 \mathrm{~nm} / \mathrm{min}$ or $0.1 \mathrm{ML} / \mathrm{min}(1 \mathrm{ML}=0.435 \mathrm{~nm})$. We may notice that the y-intercept of the fitting line is not zero. This is because in the beginning of deposition a certain mount of atoms go to the wetting layer before growing islands. Typically the coverage-deposition should be very close to linear.

The standard deviation of the fitting in Fig. 11 can be calculated as only 3\%, but the error of the calibration comes mainly from the STM system. We know when the tip scans 
along the surface, if the altitude of the surface has a significant change (e.g. from the top of an island to the wetting layer), the system can't move the tip to the proper altitude instantly, and it takes sometime to adjust the position of the tip. As a result, there seems a ramp surround each islands even if the islands are actually very steep. In these images, the lateral length of a ramp is usually 7-9 nanometers and when we calculate the total volume of islands, we integrate assuming the ramp is real. For a typical island with size $\sim 60 \mathrm{~nm}$ (fig.12) and ramp width $\sim 5 \mathrm{~nm}$, the area of the ramp around it is about $\left(60^{2}-55^{2}\right) / 60^{2}=16 \%$ of its total area, so the error due to this effect could be $15 \%$ or larger. Therefore we can estimate that the flux rate as $0.1 \pm 0.01 \mathrm{ML} / \mathrm{min}$.

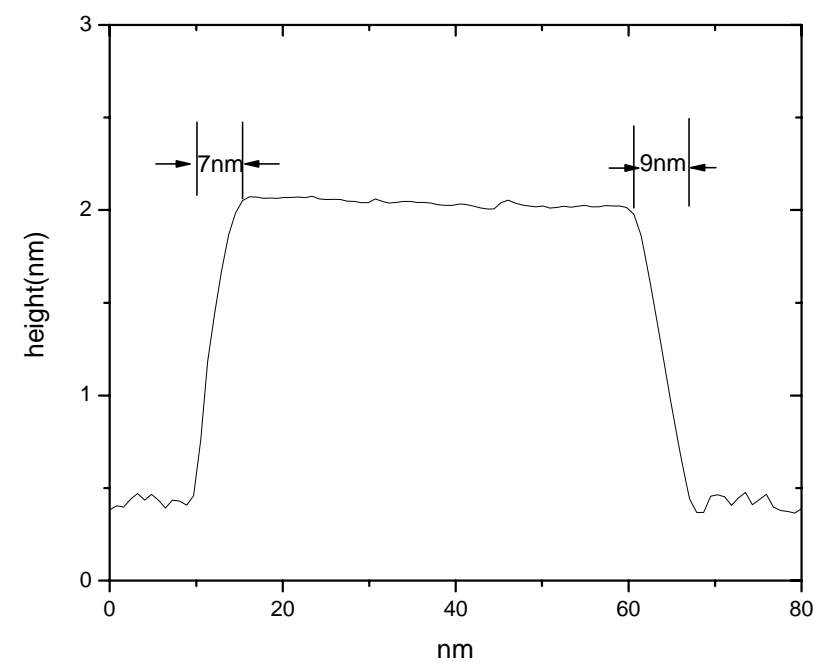

Figure 12 The cross section of a typical In island. The lateral length of its two edges are $7 \mathrm{~nm}$ and $9 \mathrm{~nm}$, respectively. The edges are not absolutely vertical due to the limited responding time of the tip.

Another method to obtain the total volume is to measure island by island. Since we know the height of every point in the STM image, we can draw contour lines on the STM images and find the area for each island by select a proper threshold value. (e.g. in Fig.13, the points higher than the threshold value is white, others are black). The area of each island is obtained by counting the number of pixels in the respect island using software. The height of each island can measured from the 1-D profiles(e.g. fig.12). The volume of each island is then the product of its area and height. 


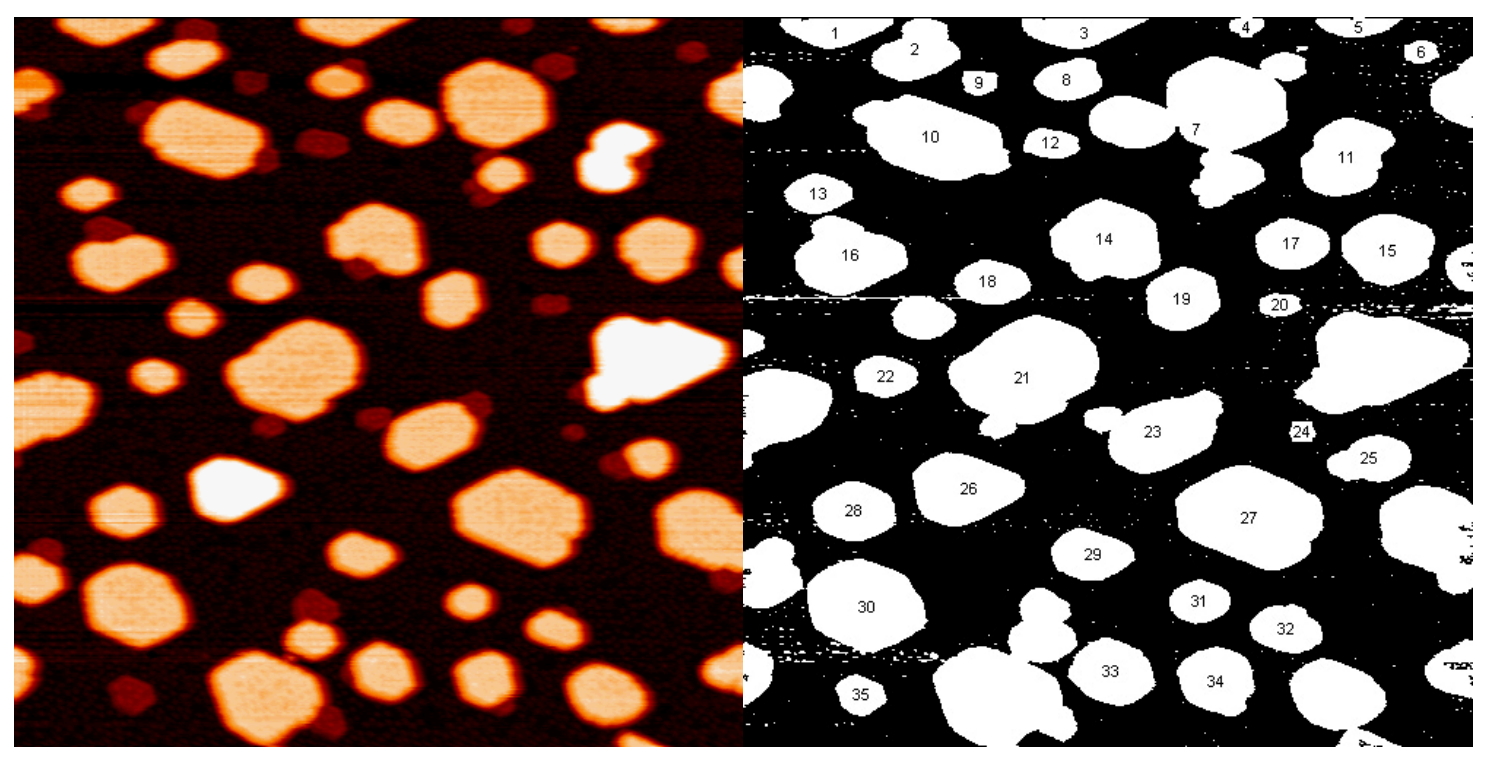

Figure 13 An STM image(200nm*200nm) and its corresponding contour line

For an example, the triangle-shaped island(\#26) in Fig. 13 contains 2800 pixels

(which is counted by software) and the image is $200 \mathrm{~nm} \times 200 \mathrm{~nm}$ and its resolution is $500 \mathrm{x}$ 500 , so each pixel corresponding to $0.16 \mathrm{~nm}^{2}$, and the area of the islands is $448 \mathrm{~nm}^{2}$, and the height of the island is $1.16 \mathrm{~nm}$ therefore its volume is $519.7 \mathrm{~nm}^{3}$. And the total coverage is the sum of the volume of all islands divided by the area of the image. In this method the main error comes from the selection of the threshold level. Theoretically the islands are sharp and the threshold should not affect the measurement but real islands are not ideal. For an example, in Fig. 12 is a cross section of a typical flat island. If the threshold level is chosen at near the top and bottom of the island height, separately, its size will differ by $7+9=16 \mathrm{~nm}$, which is more than $15 \%$ of the size of the island. The absolute error is in the same level for all the islands, so the relatively error is especially large for small islands. Another disadvantage is that the area of each islands is measured separately so it is much time-consuming. What more, it ignored the atoms between islands, which makes the coverage smaller than actual value.

Comparing the two methods, the first one measures the whole image in the same time and greatly reduced the random errors so its result is more reliable as long as the background is correctly subtracted. Correctly subtracted background means the wetting layer plane must be horizontal without tilt. It is obviously if the plane is tilted the histogram will become meaningless. 
Although STM is a very powerful tool, it shortcoming is also obvious. It can only scan a very small area (usually less than $1 \mu \mathrm{m}^{2}$ ), so it is hard to get good statistics over a large scale. Sometime if one observes a "not representative area", it may even get misleading results. On the other hand, because we need tunneling current, it is limited to the study on conductor or doped semiconductor surface. Another draw back is that it can not scan during depositions to make real time measurement like LEEM or RHEED. In many cases another instrument is required to help STM to overcome the shortcomings.

Low energy electron diffraction (LEED) is a very widely used technique. In an LEED a beam of electrons (typically $20-200 \mathrm{eV}$ ) is incident toward the surface. The wavelength of the electrons is in the same order to the lattice constant of surface, therefore the crystal diffraction can be observed. In a conventional LEED system, the diffracted electrons are scattered towards a screen and can be recorded by a camera. The diffraction pattern can be used as an auxiliary tool to judge the quality of the crystal surface roughly.

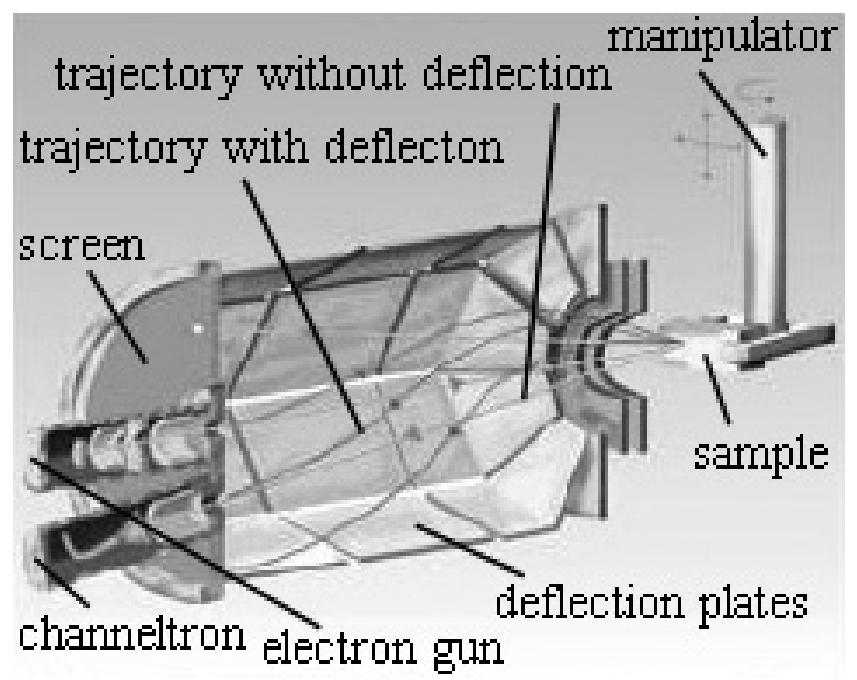

Figure 14 A schematic view showing how SPA-LEED works. Image from[5]

To do more accurate analysis in the diffraction pattern, we need to improve the resolution in reciprocal space. One solution is spot-profile analysis LEED (SPA-LEED). ${ }^{[6]}$ (Fig.14) In a SPA-LEED system, the electron beam from the electron is incident to the surface of the sample and reflected back into the channeltron. The channeltron is behind a small hole so only electrons in a specific direction, i.e., a specific position in the diffraction pattern can be detected. To get the information of the whole diffraction pattern, a pair of deflection plates are introduced to generate an octopole electron field in the chamber. By 
adjusting the octopole electron field, the electrons in all the directions can come into the channeltron, therefore, we can perform a scan in the reciprocal space by adjusting the voltage of the deflection plates. The voltage of deflection plates is controlled by a computer.

The sensitivity of the instrument in reciprocal space is determined by the transfer width of the incoming electron beam. For a conventional LEED the transfer width is typically in the order of $300 \AA$, which gives the resolution of 1-2\% Si Brillouin zone (BZ) in reciprocal space. In a SPA-LEED system, thanks to the high sensitivity of the detector, the transfer width is improved to $2000 \AA$, which provides a resolution in reciprocal space of $0.2 \% \mathrm{Si} \mathrm{BZ}$, which is one order higher than a conventional LEED. The detector can also give quantitative intensity of each point in the reciprocal space, while a conventional LEED is unable to do so or doesn't have good accuracy. The accurate positions in reciprocal space and the good spot intensity resolution allow the analysis of spots profiles which is the main investigation tool in this thesis.

When we scan in the reciprocal space, we can choose the scan of the whole 2-D surface or scan along a line. By performing a 2-D scan, we can reproduce the diffraction pattern as seen in the screen of a conventional LEED and have an overview of the information of the whole Brillouin Zone. If choosing 1-D scan we can get the intensities in a specific direction. Because the scanned area in the reciprocal space is much smaller, the time interval for each point can be increased significantly and therefore we can reduce the noise caused by random intensity fluctuation. If necessary we can perform long time scanning and get diffraction patterns with high quality. It is common to perform a 2-D scan to get a general idea about the surface and then take 1-D scans to get information with better resolutions.

Because the displacement of the electron beam and the bias voltage of the octopole are not perfectly linear, both the positions of the 1D and 2D spots need to be calibrated. Si (111) $7 \times 7$ reconstruction is a perfect reference for calibration. The positions of $\mathrm{Si} 7 \times 7$ spots are well known as $1 / 7,2 / 7 \ldots$ of $100 \% \mathrm{BZ}$, we can make a table to list the position of the spots in raw data and the corrected value. The calibration of other spots can be accomplished by linear interpolation between two $7 \times 7$ spots. For an example, In a 1-D scan, the reading for $\mathrm{Si}$ (7x7) $7 / 7$ and $8 / 7$ spots are:-96.8\% and $-109.3 \%$ in [1_10] direction, while their actual positions are $-100 \%$ and $-114.3 \%(-8 / 7 * 100 \%)$, respectively. For an In spot at $-110.6 \%$ near 
the $8 / 7$ spot, its real position can be calculated using linear interpolation: -[(110.6$109.3) /(109.3-96.8)+8] / 7 * 100 \%=-115.7 \%$.

Another useful tool to analysis date acquired in SPA-LEED is the g(s) curve. ${ }^{[7]}$ It is a method that can be used in case of islands size comparable with the transfer width of the instrument. As the energy of the electron beam varies, its momentum, therefore the normal momentum transfer component $\mathrm{K}_{\mathrm{z}}$ changes as well. The phase difference between scattering from the top of the islands and the surrounding area will vary, as a result. For certain energies, the phase difference is $0,2 \pi, 4 \pi \ldots$ In these conditions (in-phase condition) the (00) intensity in the 1D scan profile is at a maximum and has instrumentally limited full-width-at-halfmaximum (FWHM). On the other hand, for certain energies, the phase difference is $\pi, 3 \pi$, $5 \pi$. . In these conditions (out-of-phase condition) there is destructive interference between the top of the islands and the surrounding region. The intensity of (00) spot in the 1D scan profiles then have a minimum intensity and larger FWHMs. For the in-between energies, the (00) profiles can be decomposed into a narrow $\left(\mathrm{a}_{0}\right)$ and a broad component $\left(\mathrm{a}_{1}\right)$ (Fig.15).

The $g(s)$ as a function of the scaled normal component momentum transfer $s=\left(K_{z}\right.$ $/(2 \pi / d))$ is defined as the ratio of the area of the narrow component $\left(a_{0}\right)$ to the total area. $\left(a_{0}+a_{1}\right)$ ( $d$ is the single step height of the islands grown on the surface.) For an ideal situation that the islands covered exactly $50 \%$ of the area, $\mathrm{a}_{0}$ should vanish at out-of-phase conditions and $\mathrm{a}_{1}$ should vanish at in-phase conditions. The value of $g$ oscillates as the energy of the electron changes. If islands have $\mathrm{m}$ layers, i.e., its height $\mathrm{H}=\mathrm{md}$ then the oscillation period in $\mathrm{s}$ decreases, since it is given by

$$
\Delta \mathrm{s}=\Delta \mathrm{Kz} /(2 \pi / \mathrm{d})=(2 \pi / \mathrm{H}) /(2 \pi / \mathrm{d})=\mathrm{d} / \mathrm{H}=1 / \mathrm{m}
$$



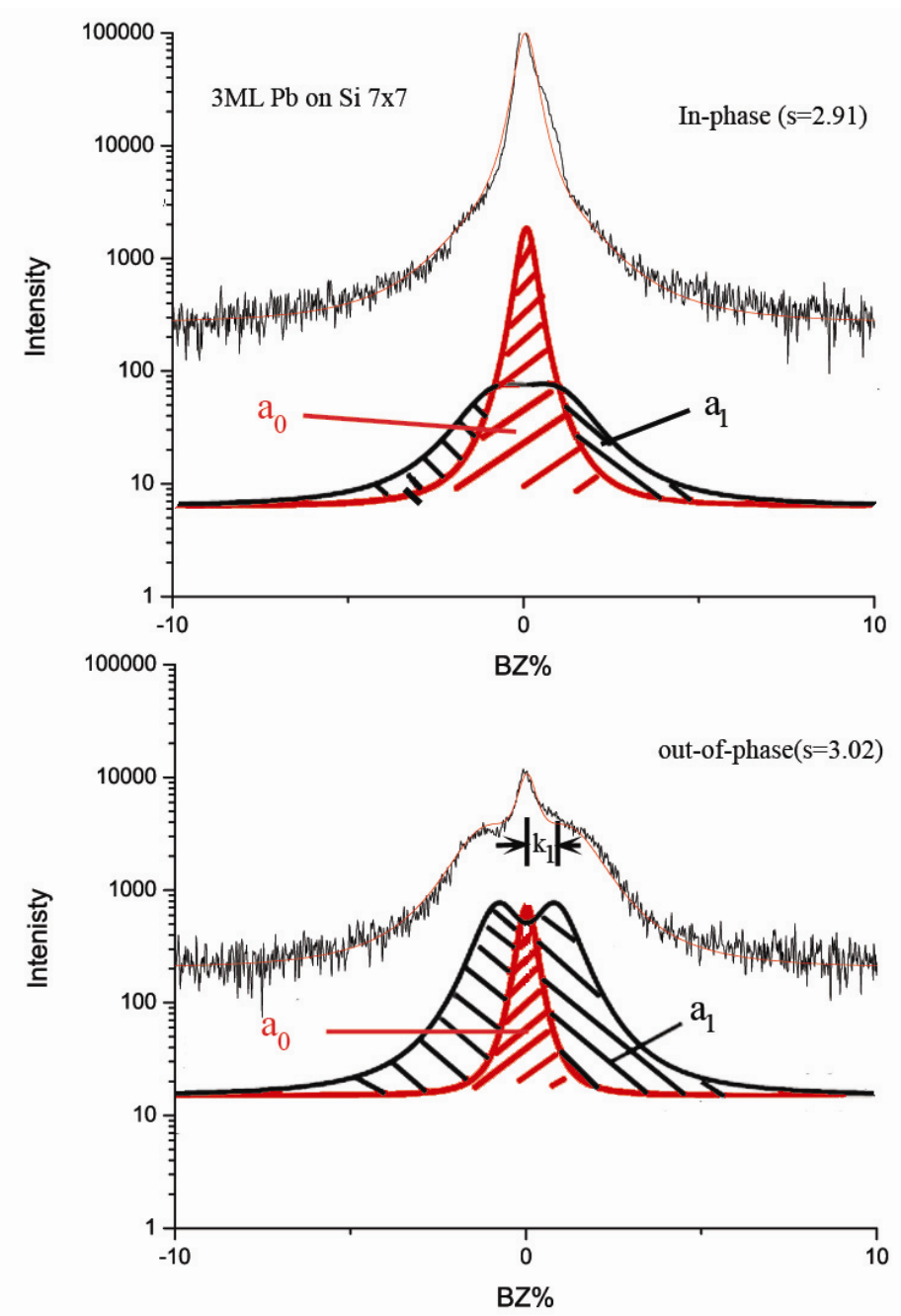

Figure 15 components of the (00) spot. The 1D profile is taken from $3 \mathrm{ML}$ of $\mathrm{Pb}$ on $\mathrm{Si} 7 \mathrm{x} 7 \mathrm{system}$ at $\mathrm{T}=-$ $185 \mathrm{~K}$ in [1_10] direction. The (00) spot can be decomposed into two parts. ${ }^{[7]}$ The red part $\left(\mathrm{a}_{0}\right)$ is the narrow part and the black part $\left(a_{1}\right)$ is the broad part. In in-phase condition, $a_{0}$ part is big and $a_{1}$ part is small, while vice-versa in out-of-phase condition.

So the period of the oscillations of the $\mathrm{g}(\mathrm{s})$ curve is inversely proportional to the island height. Therefore we can deduce the number of layers in the islands from the period. Fig.16 gives an example of $g(s)$ curve. The upper-left part is the $g(s)$ curve, which shows the 7 -fold oscillation between $s=3$ and $s=4$. From the $g(s)$ curve we can make a conclusion that most $\mathrm{Pb}$ islands on the surface are 7-layers high, which is consistent with the STM experiments. The lower-left part is the $\sigma_{0}$ (a parameter in the fitting, equivalent to the FWHM) v.s. s curve. We can see it oscillates as well. In the right part we give two representative profiles which are in in-phase condition and in out-of-phase condition, respectively. From the fit curve in red we can see the strong broad component (the shoulder) in the out-of-phase 
condition. There is a factor of 1.5 in fig. 16 for $\sigma_{0}$ and $\mathrm{k}_{1}$ values, this is because we did all the fitting in 1-dimension, but these spots are actually 2-D, so the factor is used to get the correct values of $\sigma_{0}$ and $\mathrm{k}_{1}$.
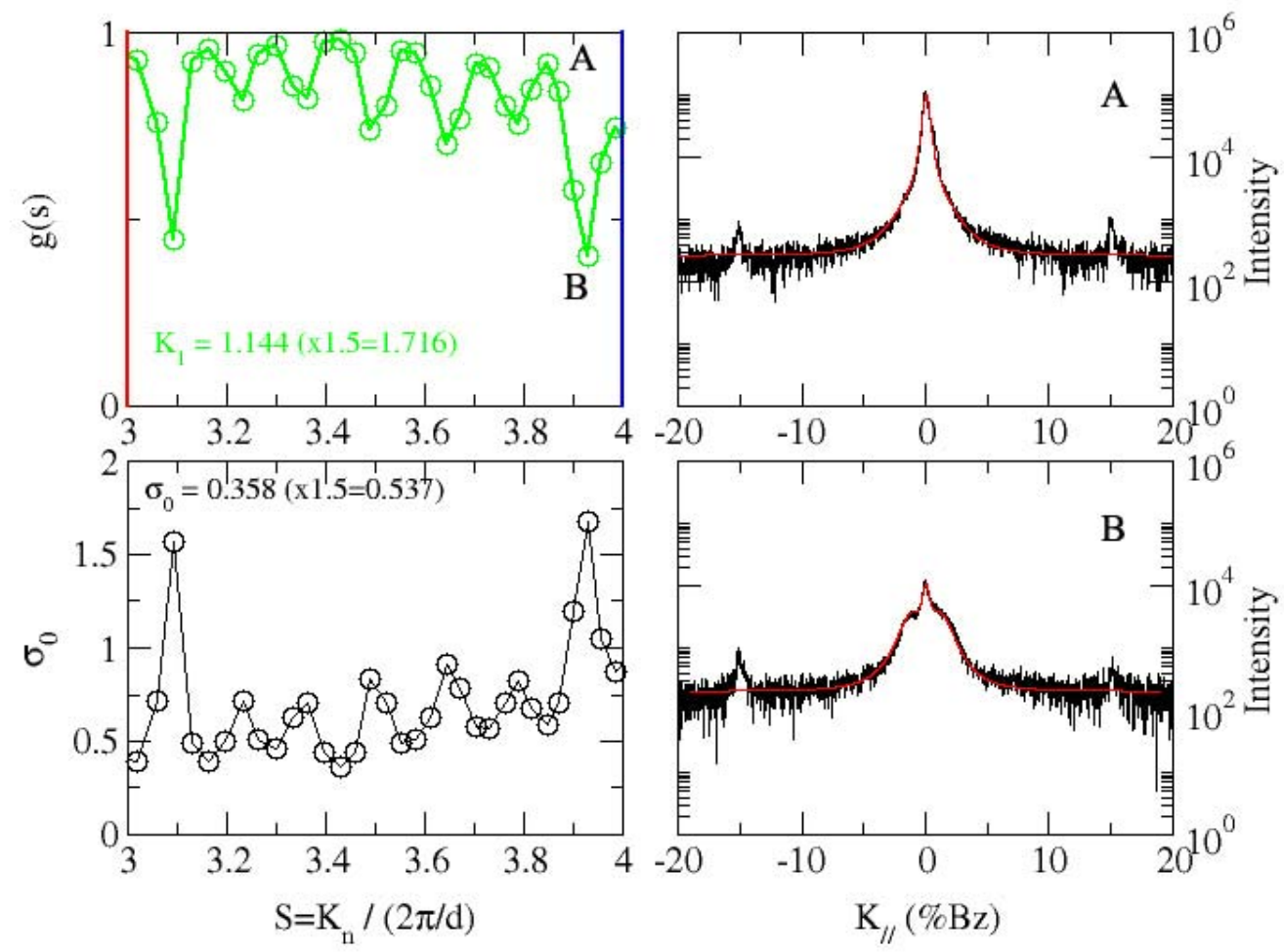

Figure 16 a $\mathrm{g}(\mathrm{s})$ curve taken from $\mathrm{Pb}$ islands grown on $\mathrm{Si}(111) 7 \times 7$ surface. $($ coverage $=3 \mathrm{ML}, \mathrm{T}=185 \mathrm{~K})$ The $K_{1}$ and $\sigma_{0}$ values have a factor of 1.5 due to the dimension concern. A, B are representative in-phase and out-of-phase profiles.

The fitting is done by a program called "gir", which uses the combination of a Gaussian function (for the narrow component) and a 3/2 Lorentzian function (for the broad component) to fit the $(00)$ profile. 


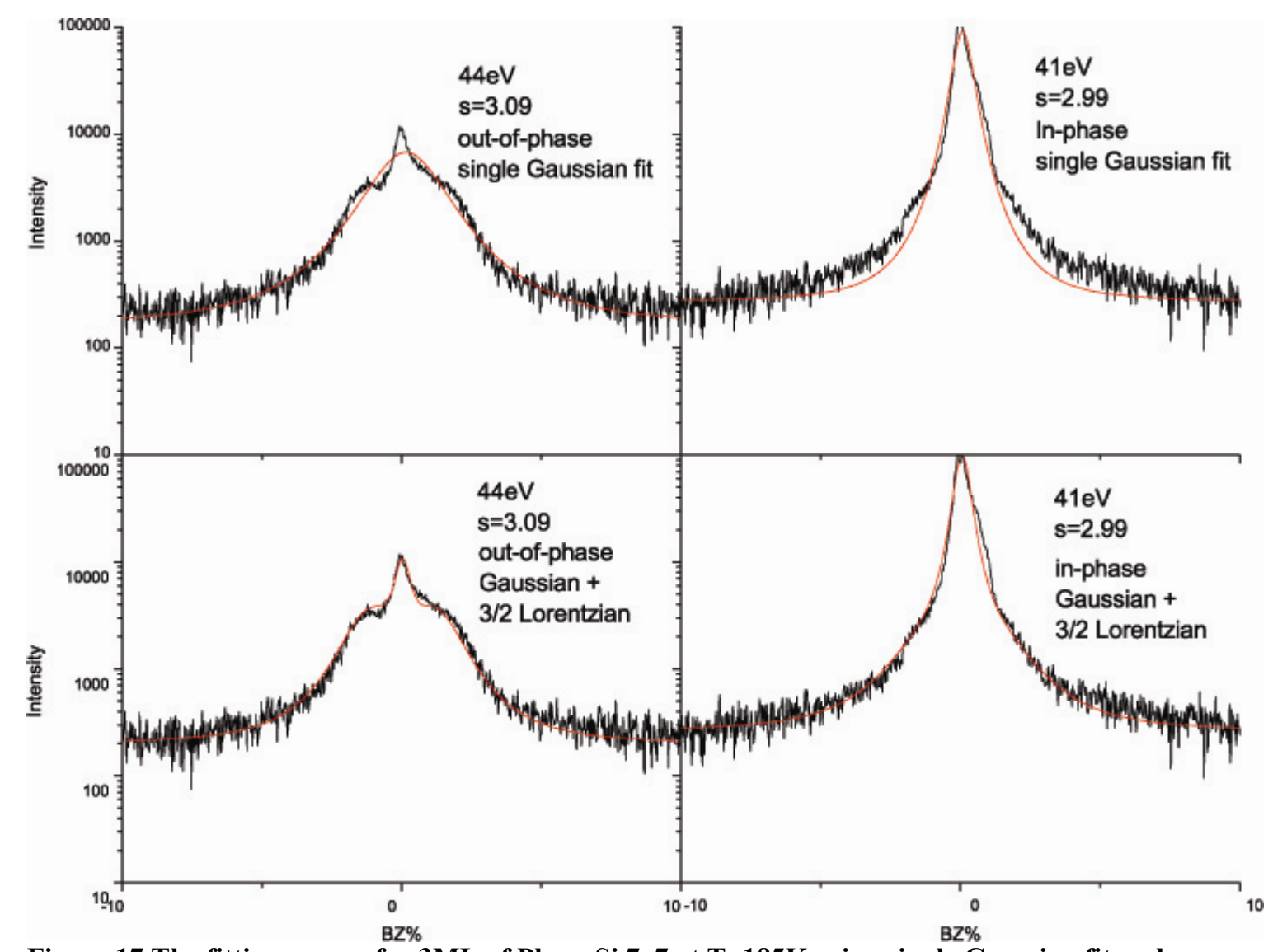

Figure 17 The fitting curves for $3 \mathrm{ML}$ of $\mathrm{Pb}$ on $\mathrm{Si} 7 \times 7$ at $\mathrm{T}=185 \mathrm{~K}$ using single Gaussian fit and Gaussian $+3 / 2$ Lorentzian, $E=41 \mathrm{eV}$ and $44 \mathrm{eV}$, separately.

The detailed process to make a $\mathrm{g}(\mathrm{s})$ curve is the following:

1. We obtain the 1-D I(intensity)-k(parallel wave vector) profiles of (00) spot at different energies (typically taking a scan every one $\mathrm{eV}$ interval and the energy range covering between $s=3$ and $s=4$, e.g. from $38 \mathrm{eV}$ to $75 \mathrm{eV}$ ) from SPA-LEED. In Fig. 17-19 the six profiles are $39 \mathrm{eV}, 42 \mathrm{eV}, 61 \mathrm{eV}, 68 \mathrm{eV}, 64 \mathrm{eV}, 71 \mathrm{eV}$, respectively.

2. Preparations before fitting. We need to check if there are abnormal points (e.g. intensity value which is several orders larger than a typical intensity value and too large to be physical), or "spikes". Those abnormal points need to be removed.

3. We fit all the I(intensity)-k(parallel wave vector) profiles using a single Gaussian function

$$
I(k)=a e^{-\frac{k^{2}}{2 \sigma_{0}^{2}}}+c
$$




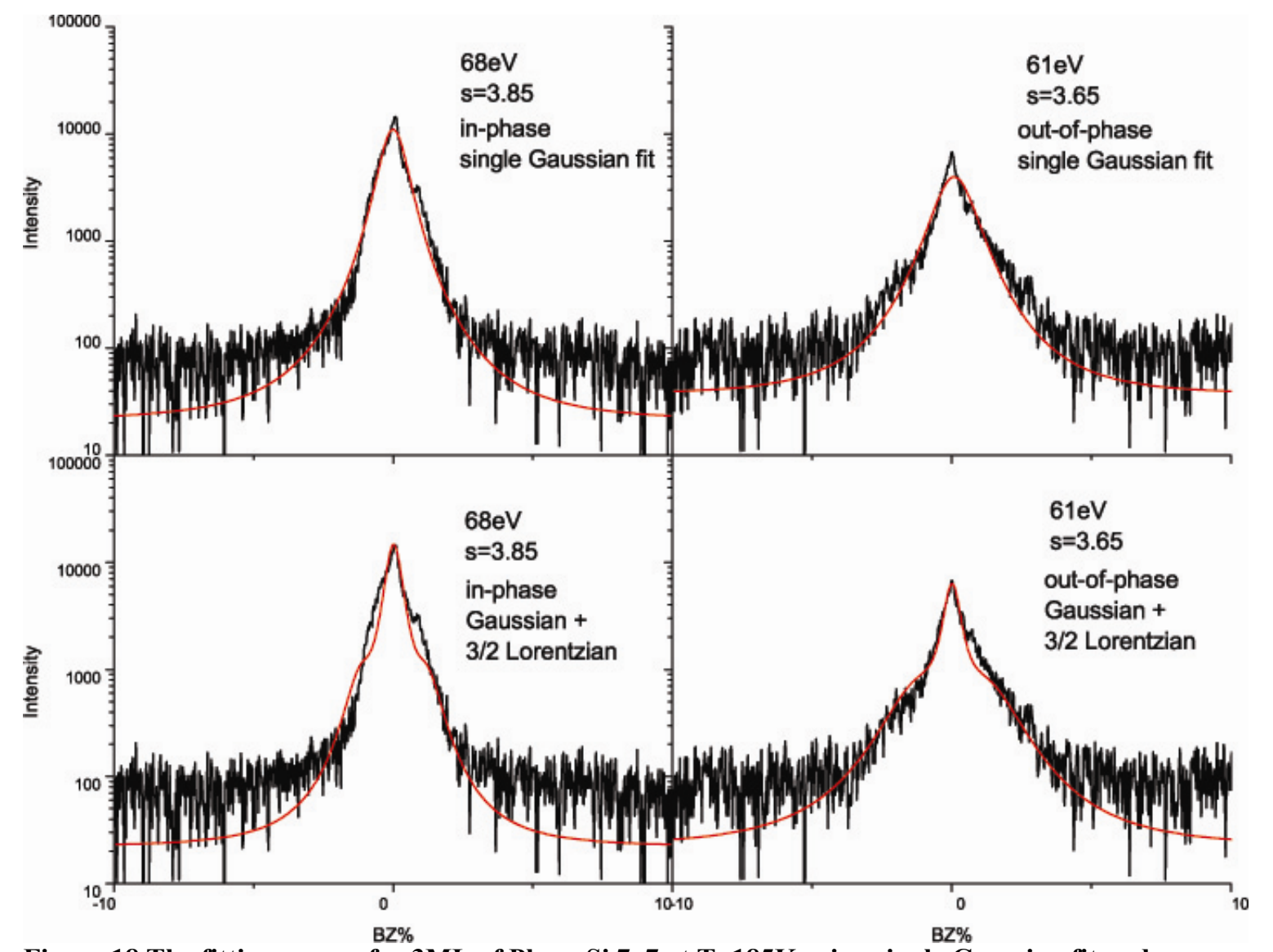

Figure 18 The fitting curves for $3 \mathrm{ML}$ of $\mathrm{Pb}$ on $\mathrm{Si} 7 \times \mathrm{x} 7$ at $\mathrm{T}=185 \mathrm{~K}$ using single Gaussian fit and Gaussian $+3 / 2$ Lorentzian, $E=61 \mathrm{eV}$ and $68 \mathrm{eV}$, separately.

(Fig.17-19 shows six representative profiles as examples.). This is done by running the program to make an Gaussian fit in the $(00)$ mode. Parameters a, c, $\sigma_{0}$ can be determined by the fitting grogram.

From the Gaussian fit we can get a $\sigma_{0}$ (width of the Gaussian curve) for each profile. In these profiles, those at in-phase conditions have smaller $\sigma_{0}$ values and those at out-ofphase conditions have larger $\sigma_{0}$ values. Therefore, the smallest $\sigma_{0}$ is the closest to the real $\sigma_{0}$ value, which corresponds to in-phase condition and there is no or very little broad component's contribution and we can determine the value of $\sigma_{0}$. 


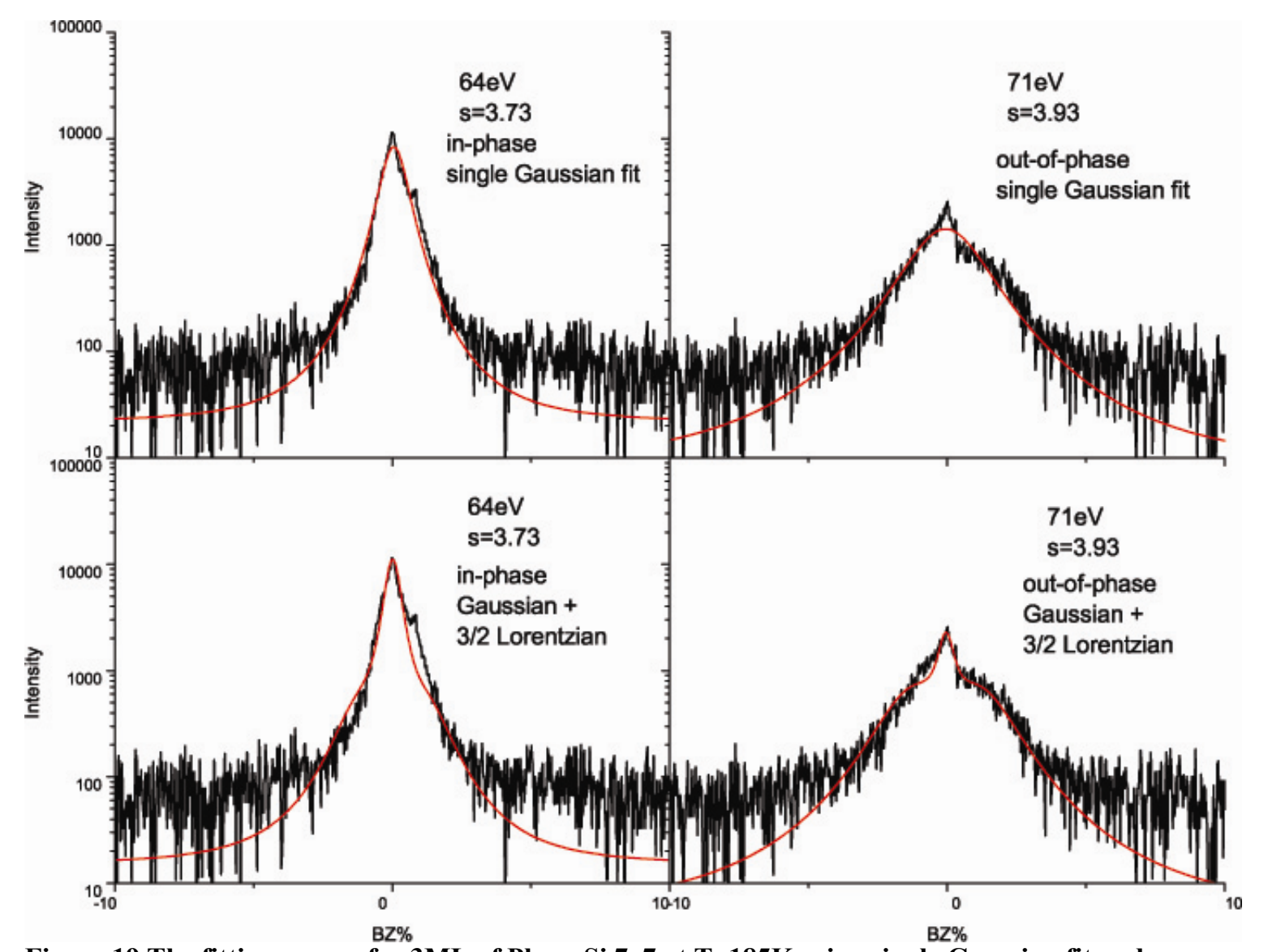

Figure 19 The fitting curves for $3 \mathrm{ML}$ of $\mathrm{Pb}$ on $\mathrm{Si} 7 \times \mathrm{x} 7$ at $\mathrm{T}=185 \mathrm{~K}$ using single Gaussian fit and Gaussian $+3 / 2$ Lorentzian, $E=64 \mathrm{eV}$ and $71 \mathrm{eV}$, separately.

4. After $\sigma_{0}$ is determined, we can pick out all the out-of-phase condition profiles (those have largest $\sigma_{0}$ values) with combination of a Gaussian function with the fixed $\sigma_{0}$ and a pair of 3/2 Lorentzian functions:

$$
I(k)=a e^{-\frac{k^{2}}{2 \sigma_{0}^{2}}}+\frac{b}{\left|k-k_{1}\right|^{3 / 2}+d^{3 / 2}}+\frac{b}{\left|k+k_{1}\right|^{3 / 2}+d^{3 / 2}}+c
$$

This fit is done by the double ring mode in the program. Parameters a, b, c, d, $\mathrm{k}_{1}$ can be determined by the program. The main purpose of this fit is to determine $\mathrm{k}_{1}$, i.e. the position of the "shoulders" for the broad components (Fig.15). The averaged $\mathrm{k}_{1}$ value from these fitting is taken as the $\mathrm{k}_{1}$ value for all the profiles in the next step because the value of $\mathrm{k}_{1}$ should not dependent on energy of electron.

5.After the two key parameters $\sigma_{0}$ and $\mathrm{k}_{1}$ are determined, we can run the fitting program again in the double-ring mode on all the profiles to get the eventual fitting curves 
with the combination of a Gaussian function with the fixed $\sigma_{0}$ and a pair of 3/2 Lorentzian functions with the fixed $k_{1}$. In Fig.17-19 the fitting curves in the bottom are shown as representative profiles. The two-component fitting is much better than the single Gaussian fitting in the upper half and very close to the raw data.

6. After the fitting is accomplished, we have obtained the area of narrow component $\mathrm{a}_{0}=(2 \pi)^{1 / 2} \mathrm{a} \sigma_{0}$ and the area of the broad component $\mathrm{a}_{1}=(16 \pi \mathrm{b} \sqrt{3}) /(9 \sqrt{\mathrm{d}})$ in each profile, and therefore we can plot the $g(s)$ curve: $g=a_{0} /\left(a_{0}+a_{1}\right)$.

Let's see a specific example. We put 1.5ML of indium on $\mathrm{Pb}$ alpha phase and then take the 1-D profiles at different energies. Fig.21 (lower-left part) shows the $\sigma_{0}$ vs s curve from step 3. The $\sigma_{0}$ value range is from 0.322 to 1.587 , so 0.322 is used as the fixed $\sigma_{0}$ in the following step. An in-phase profile and an out-of-phase profile are shown in fig.20. In step 4, we choose file $205,210,214,221,230,238$ (those have the largest $\sigma_{0}$ values) as out-ofconditions. $\sigma_{0}$ is fixed as 0.322 , and we get the fit value for $\mathrm{k} 1$ are: $3.72 \mathrm{E}+37,0.888,0.868$, $1.058,1.058,56152000$. The first and last values are unphysical and not counted, the average value of the rest four is 0.94. Fig. 21 shows the fitting curve for file 210 and 214. In step 5, the fixed parameters are $\sigma_{0}=0.322, \mathrm{k} 1=0.94$, and we get the final fitting curve. In the right side in Fig.22 are two representative in-phase and out-of-phase fitting. And we can draw the $\mathrm{g}(\mathrm{s})$ curve from the fitting (upper-left in fig.22), which gives a clear 5-fold oscillation.

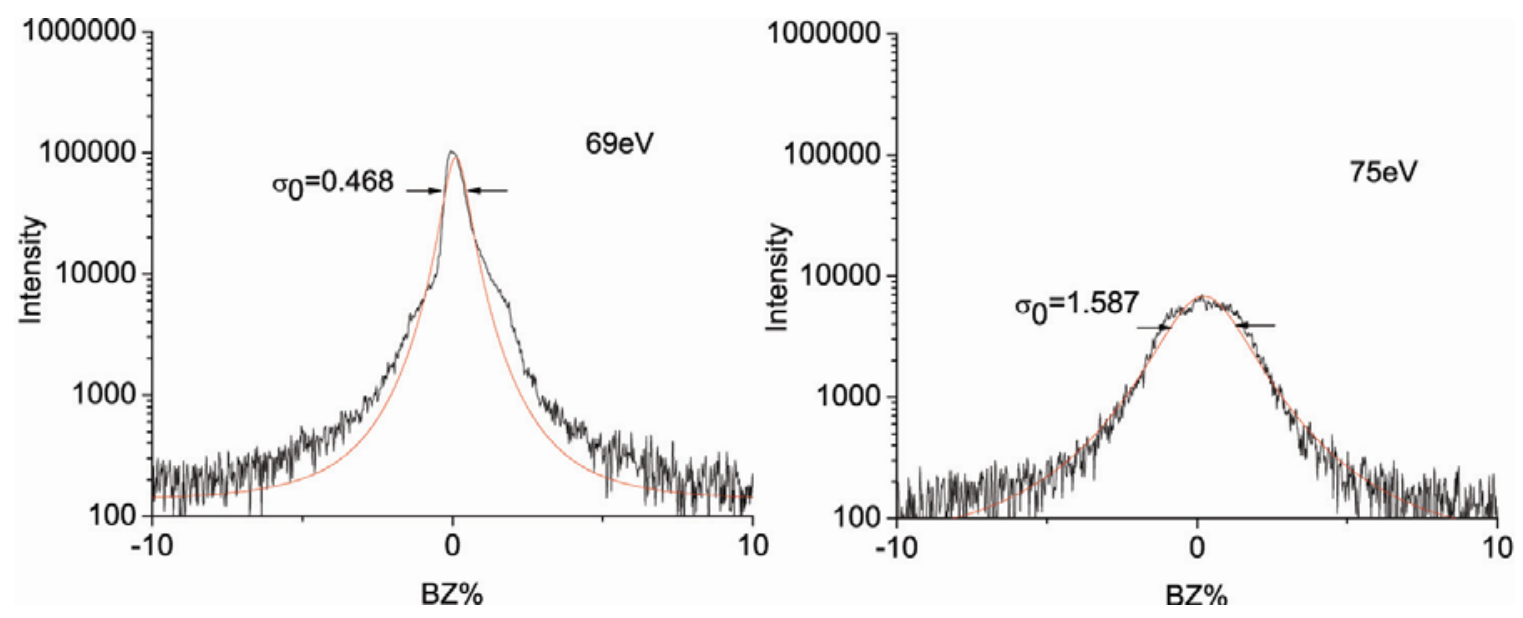

Figure 20 Single Gaussian fit for In phase $\left(\sigma_{0}=0.468\right)$ condition and out-of-phase $\left(\sigma_{0}=1.587\right)$ condition. The system is $1.5 \mathrm{ML}$ of $\mathrm{In}$ on $\mathrm{Pb}$ alpha phase, $\mathrm{T}=185 \mathrm{~K}$. 

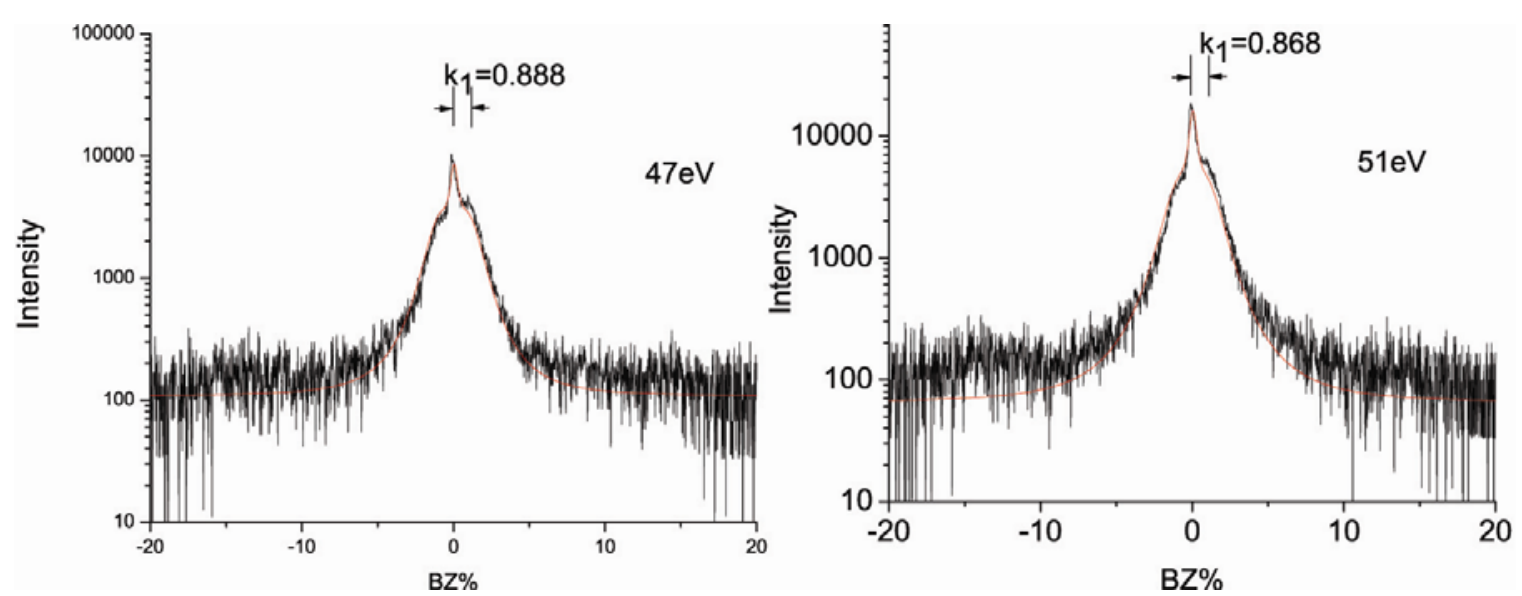

Figure 21 Two-component fit for out-of-phase condition curves. (a) $E=47 \mathrm{eV}, \mathrm{k}_{1}=0.888$ (b) $E=51 \mathrm{eV}$ $k_{1}=0.868$. The system is $1.5 \mathrm{ML}$ of $\mathrm{In}$ on Pb alpha phase, $\mathrm{T}=185 \mathrm{~K}$.

Some time the fitting curve doesn't agree with raw data very well. In these cases we need to adjust the fixed parameters manually to find the parameters that can represent the fitting curve best.
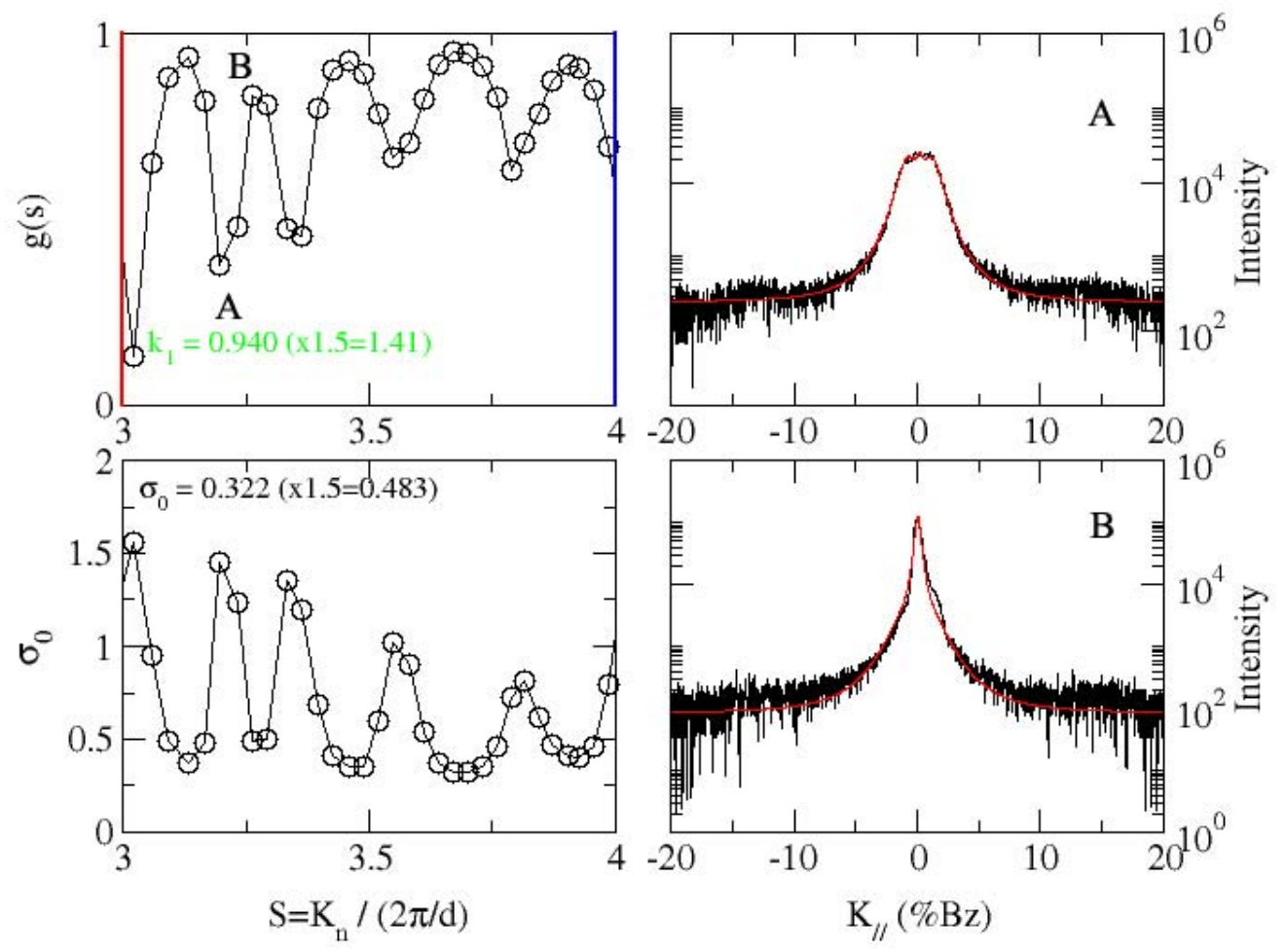

Figure $22 \mathrm{~g}(\mathrm{~s})$ curve for $1.5 \mathrm{ML}$ indium on $\mathrm{Pb}$ alpha phase at $\mathrm{T}=185 \mathrm{~K}$ (upper left). $\sigma_{0}$-s curve(lower-left) and a typical out-of-phase(upper-right) and in-phase(lower-right) profile is also included. 
The $\mathrm{g}(\mathrm{s})$ curve analysis is very useful when most of the islands are in the same height due to QSE and can be used to determine the islands height solely from diffraction without the help of the STM. However, if there is no preferred height in the islands or the islands size is too large and exceeding the instrument limit, it is hard to deduce information from the $\mathrm{g}(\mathrm{s})$ curves.
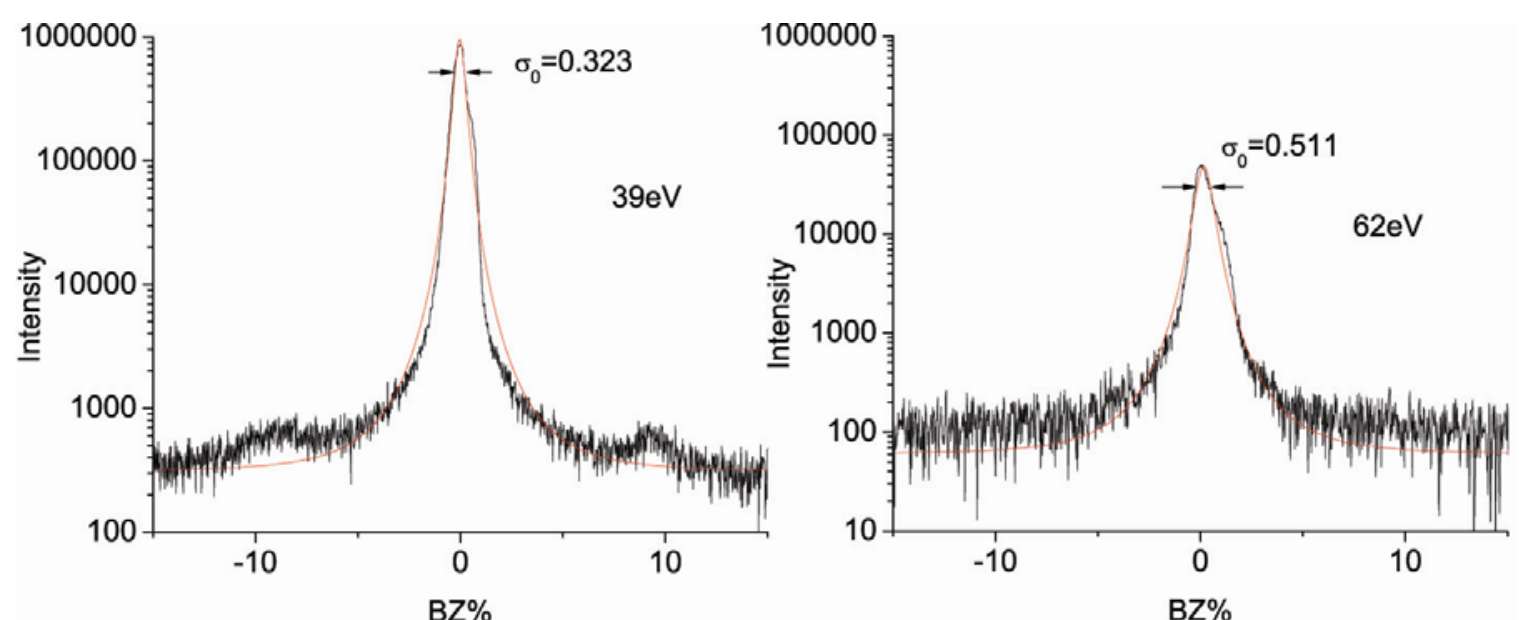

Figure 23 Single Gaussian fit for $3 \mathrm{ML}$ of In on In $4 \times 1$ phase $\left(\sigma_{0}=0.323,39 \mathrm{eV}\right)$ condition and out-of-phase $\left(\sigma_{0}=0.511,62 \mathrm{eV}\right)$ condition. The value of $\sigma_{0}$ in out-phase-condition is very small.

An example is the deposition of In on In $4 \times 1$ phase. We attempted to get the $\sigma_{0}$ vs $\mathrm{s}$ curve when $3 \mathrm{ML}$ of In are deposited onto the In 4x1 phase at T=170K in Fig.23. (see Ch.6 for more detailed discussions) The values $\sigma_{0}$ are very small, which indicates that there is no significant difference between in-phase and out-of-phase conditions from the profiles. The extremely small $\sigma_{0}$ values $(<0.5 \%)$ means that the islands sizes are more than $700 \AA$ and are very close to or have exceeded the limit of instruments. Therefore we can not get a $\mathrm{k}_{1}$ value with physical meaning. In this situation there is no significant difference between in-phase conditions and out-of-phase conditions.(Fig.23) Even if we force the program to finish the rest steps and get a g(s) curve(Fig.24), we cannot get much useful information. And it is not possible to find the height of islands via $g(s)$ curve in the case. This example shows that the method of $g(s)$ curve has its limitation. In a summary, if $\sigma_{0}$ are too small and close to the limit of instrument, it is not reliable any more. In these situations, $\mathrm{g}(\mathrm{s})$ curve is not a tool to study the properties of islands. 

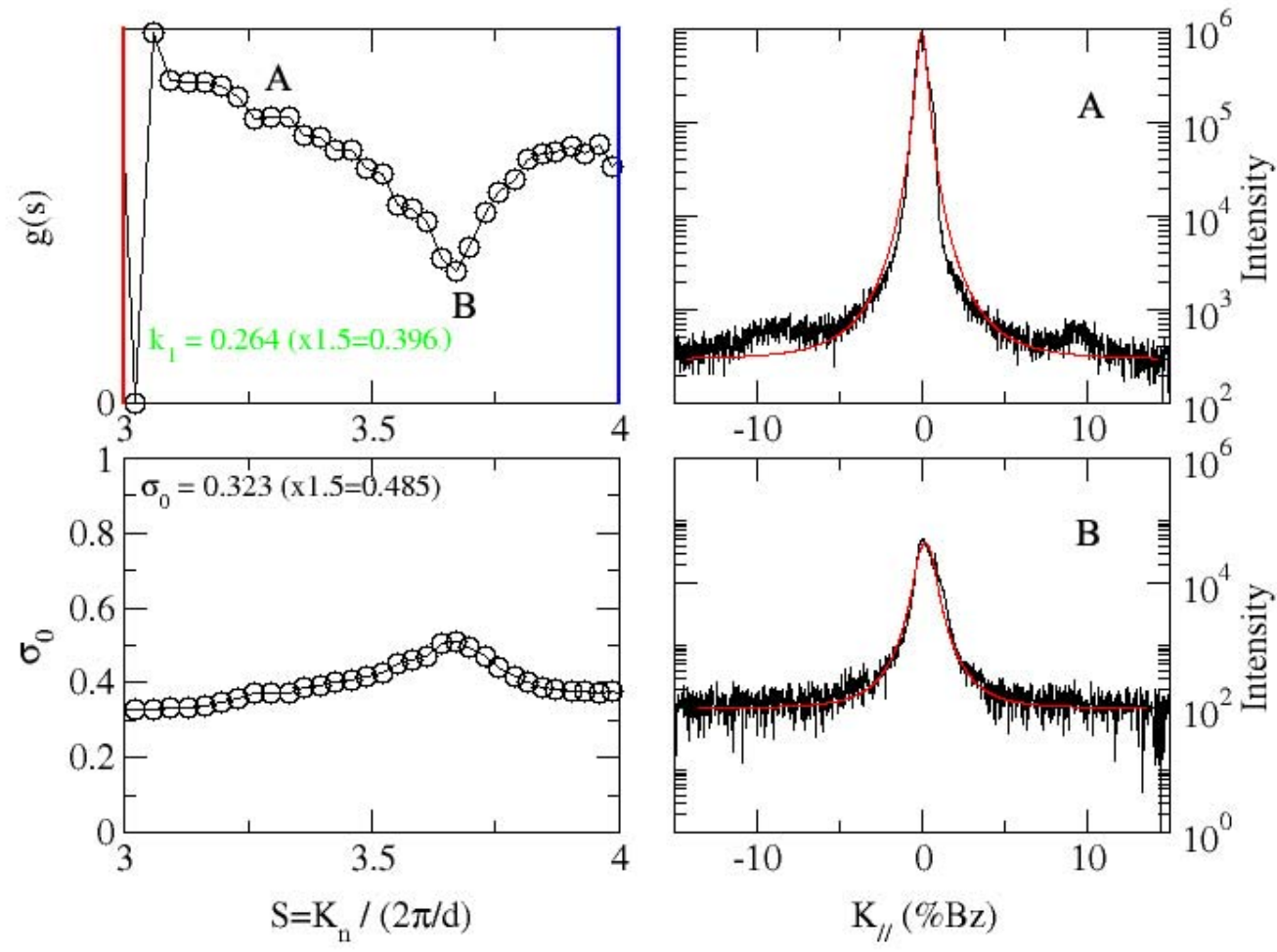

Figure $24 \sigma_{0}$ vs s curve when In islands grows on In $4 \times 1$ phase.

References:

[1] B.S. Swartzentruber, et al., J. Vac. Sci. Technol. A 7, 4(1989)

[2] M. Hupalo, et al., Phys. Rev. Lett. 90, 216106 (2003)

[3] M. Yakes, et al., Phys. Rev. B 69, 224103 (2004)

[4] http://www.omicron-instruments.com/ltstm/STM.html

[5] http://www.physik.fu-berlin.de/ ag-rieder/spaleed.html

[6] U. Scheithauer, et. al., Sci. Surf. 178,441(1986)

[7] S. Stepanovskyy, et.al, Sur. Sci. 515,187(2002) 


\title{
CHAPTER 3: UNUSUAL THERMAL STABILITY OF QUANTUM SIZE EFEECT PB ISLANDS GROWN ON SI(111)-IN(4x1)
}

\author{
A paper published in App. Phys. Lett., 90, 163117 (2007) \\ M.Yakes ${ }^{1}$, J. Chen ${ }^{2}$, M. Hupalo ${ }^{3}$, and M.C. Tringides ${ }^{4}$
}

\begin{abstract}
$\mathrm{Pb}$ islands grown on the anisotropic $\mathrm{Si}(111)-\operatorname{In}(4 \mathrm{x} 1)$ were studied using STM and SPA-LEED. Anisotropic wire shapes with a uniform island height of 4 layers due to quantum size effects are observed as well as a preferred width of $6.6 \mathrm{~nm}$. Unlike islands grown on clean and $\mathrm{Pb}$ covered $\mathrm{Si}(111), \mathrm{Pb}$ islands grown on the $\mathrm{Si}(111)-$ $\operatorname{In}(4 \times 1)$ reconstructed surface maintain 4 layer heights to room temperature. The increased temperature stability enhances the potential use of the grown structures to technological applications.
\end{abstract}

In order to fully utilize the potential of self-assembled nanostructures, it will be important to fabricate structures that have uniform size and geometry. A physical phenomenon that has great promise as a tool in assembling uniform height structures is the quantum size effect (QSE) i.e. the dependence of the electron confinement energy on structure height. ${ }^{[1]}$ Recently, the $\mathrm{Pb} / \mathrm{Si}(111)$ system has shown the best evidence that not only QSE are observable in nanostructures, but also because of the variation of the electronic energy with height an extremely sharp height distribution is obtained. The sharpest height distribution for growth on $\mathrm{Si}(111)-7 \times 7$ was found to be 7 layer islands grown at $\sim 180 \mathrm{~K}$. This is due to both the energy ${ }^{1}$ minima vs height of the confined electron energy, but also due to kinetics, i.e. this is the best temperature to build the uniform height islands within the deposition time. These results have been confirmed with a variety of techniques including high resolution Electron Diffraction, STM, STS, X-ray scattering, and ARPES. ${ }^{[2-6]}$ Despite the great success of QSE in $\mathrm{Pb} / \mathrm{Si}(111)$, one caveat is that these structures are metastable and

\footnotetext{
${ }^{1}$ Graduate student, primary researcher

${ }^{2}$ Graduate student, participated in data analysis

${ }^{3}$ Staff scientist, conducted STM experiments

${ }^{4}$ Professor, author of corrspondence
} 
with mild $(\sim 50 \mathrm{~K})$ thermal annealing they transform into larger heights, so the mono-disperse distribution is lost. For these islands to have potential technological applications it is important to find ways to preserve the height uniformity to room temperature. This is possible with the use of foreign adsorbates (surfactants) or by growing on other interfaces. ${ }^{[7-9]}$. As reported earlier with $\mathrm{STM} \mathrm{Pb}$ grown on $\mathrm{Si}(111)-\operatorname{In}(4 \mathrm{x} 1)$ shows extended stability. In addition the combined effect of QSE and anisotropic strain, due to the underlying anisotropic reconstruction, results in elongated islands of controllable height and width. ${ }^{[10]}$ The preferred height is 4 layers with respect to the $\operatorname{In}(4 \times 1)$ substrate and the preferred width is $5 \mathrm{w}_{0}$, where $\mathrm{w}_{0}=1.35 \mathrm{~nm}$ is the width of the $4 \times 1$ unit cell along the [11 $\left.\overline{2}\right]$ direction (after growing $3 \mathrm{ML}$ at $180 \mathrm{~K}$ ). The 4 layer height was also the preferred height for growth on a different reconstructed phase $\mathrm{In}-\sqrt{3} 1 \mathrm{x} \sqrt{3}$, thus indicating unusual stability for the 4 layer $\mathrm{Pb}$ islands on $\mathrm{Si}(111)$ when covered with In.

In this work we have conducted systematically thermal annealing experiments of $\mathrm{Pb}$ islands grown on the In $(4 \times 1)$ structure to determine changes on the island dimensions, by performing parallel STM and SPA-LEED measurements. Because of the large area probed in diffraction (which includes more than $10^{5}$ islands) these experiments provide excellent statistical information about the thermal stability of the $\mathrm{Pb}$ islands. ${ }^{[1]}$ Experiments took place in two separate UHV chambers with experimental setup and $\operatorname{Si}(111)-\operatorname{In}(4 \times 1)$ preparation method as described elsewhere. ${ }^{[2,10]} \mathrm{Pb}$ islands were prepared by depositing 2.5$3 \mathrm{ML}$ of $\mathrm{Pb}$ at $0.1-0.2 \mathrm{ML} / \mathrm{min}$ at $185 \mathrm{~K}$. After deposition the crystal was annealed incrementally to higher temperatures.

Fig. 1 shows STM images taken at $185 \mathrm{~K}$. Figure 1(a) shows a $100 \times 100 \mathrm{~nm}^{2}$ area of 2.3 $\mathrm{ML}$ of $\mathrm{Pb}$ grown on $\mathrm{In}(4 \mathrm{x} 1)$. At this coverage $2 \mathrm{ML}$ of $\mathrm{Pb}$ form the wetting layer and the remaining 0.3 ML forms islands of 2layers (4 layers total as measured from the substrate), giving total area covered by the islands $15 \%$. Between the anisotropic $\mathrm{Pb}$ islands the second layer is seen to be incomplete with the exposed trenches that descend to the first $\mathrm{Pb}$ layer. Figure 1(b) shows a $200 \times 200 \mathrm{~nm}^{2}$ area after overnight RT anneal. As seen in Fig. 1(b) the wetting layer between the islands is smoother and the uncovered area is reduced indicating that most of the top layer of the wetting layer has moved to the islands. The 1-D scans shown underneath each figure along the direction of the white lines in the images confirm 
that the island height is unchanged after annealing: it is $0.61 \mathrm{~nm}$ at $185 \mathrm{~K}$ (corresponding to 4layers from $\operatorname{In}(4 \times 1)$ if the 2 layers in the wetting layer are added) and $0.99 \mathrm{~nm}$ at $\mathrm{RT}$ (corresponding to 4-layers from $\operatorname{In}(4 \mathrm{x} 1)$ if the remaining 1-layer in the wetting layer is added).

$40 \% \times 40 \%$ silicon Brillioun Zone (BZ) 2-D scans around the (00) spot are shown in Fig. 2. The temperature of each scan is labeled in the figure. The spot profile of the specular beam consists of a sharp central spike due to the long range order on the surface and a broad component due to correlation of the lateral separation between $\mathrm{Pb}$ islands ${ }^{[2]}$. Due to the anisotropic size and separation between the $\mathrm{Pb}$ islands, the satellite ring is not isotropic but instead is elongated along [1 $\overline{1} 0]$, the direction of the shortest island dimension. The 3 -fold symmetry of the $\operatorname{Si}(111)$ surface and the three possible directions of the growth of the $\operatorname{In}(4 \times 1)$ chains give the 6 arms seen in the diffraction pattern. As the temperature increases, the intensity of the central spot increases (which also confirms the transfer of $\mathrm{Pb}$ from the top layer of the wetting layer) and the length of the arms decreases (because of coarsening of the island lateral dimensions). With increasing temperature there are strong spots near $11 \% \mathrm{BZ}$ and weaker spots near $15 \% \mathrm{BZ}$ along the $[1 \overline{1} 0]$ direction, which come from the corrugation on the island tops due to the lattice mismatch between the $\mathrm{Pb}$ islands and the underlying $4 \mathrm{x} 1$ substrate $^{[13]}$. This indicates, as in all the other interfaces studied so far that the $\mathrm{Pb}$ islands are crystalline through the wetting layer down to the $\mathrm{Si}$ substrate and that the height is unchanged (since the corrugation intensity decreases with increasing island height). 


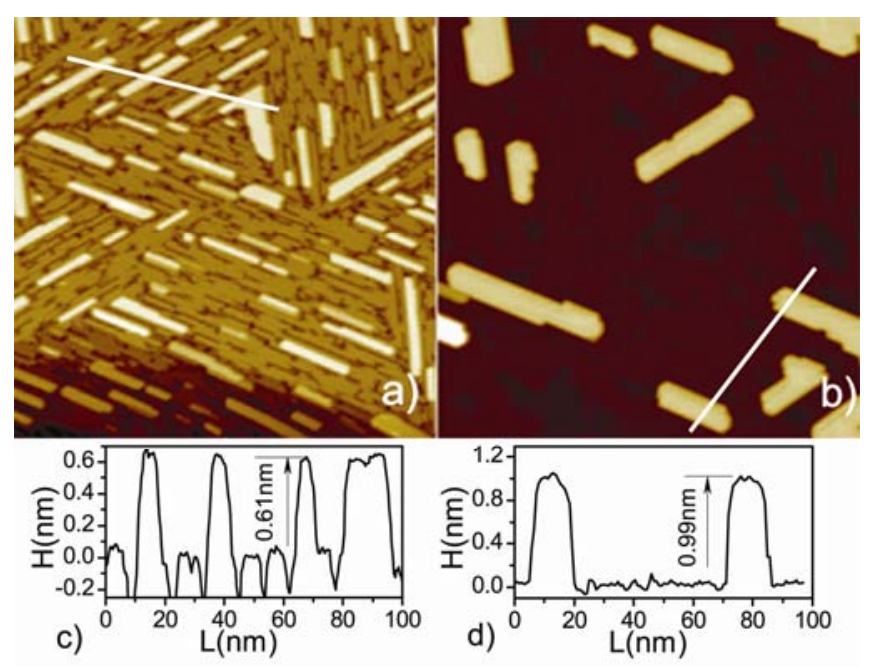

Figure 1 (a) $100 \times 10 \mathrm{~nm}^{2}$ image showing 2.3 ML of Pb deposited on the Si(111)-In(4x1) phase. 4 layer nanowires are present separated by 2 layer wetting layer. (b) $200 \times 200 \mathrm{~nm}^{2}$ scan after annealing to RT. The 1-d scans below each image and the histograms ( not shown) confirm that the islands still have the same 4 layers when measured from $\operatorname{In}(4 \times 1)$.

With SPA-LEED, when the terrace width is comparable to the transfer width of the instrument, the surface morphology can be investigated using G(s) curves. The function G(s) is the ratio of the sharp to the broad component of the spot profile with changing momentum transfer. The momentum transfer is normalized to the $\mathrm{Pb}$ step height as a function of the momentum transfer normal to the surface $\left(s=k_{\perp} /(2 \pi / d)\right.$, where $d$ is the layer height of $\mathrm{Pb}(111))$. Minima of the $\mathrm{G}(\mathrm{s})$ occur when the diffracted wave from the island tops and wetting layer are out-of-phase and maxima occur when the waves are in phase. Typical in phase and out of phase profiles are shown in the bottom right Fig. 3. The period of the oscillations of the G(s) curve is inversely proportional to the island height. Fig, 3 shows the $\mathrm{G}(\mathrm{s})$ curves for 5 temperatures. In each case four oscillations are clearly seen in a single period of the normalized momentum transfer. At the lowest temperature the last two oscillations are suppressed. At this temperature for electron energy above $60 \mathrm{eV}(\mathrm{s}>3.6)$ the scattering factor is so low that the separation of the profile into broad and narrow components is difficult. At $215 \mathrm{~K}$ and $242 \mathrm{~K}$ the oscillations are very strong, and then become weaker at $265 \mathrm{~K}$ and $285 \mathrm{~K}$, though the oscillations are still clearly seen at the Fullwidth-half-maximum (FWHM) vs s (not shown). This is simply due to the island size becoming a larger fraction of the coherence length. Also shown is the $\mathrm{k}_{1}$ value, the wavevector of the parallel component of the momentum transfer at the satellite rings (shown at the bottom right Fig.3) which corresponds to the most probable separation between islands. 
At $185 \mathrm{~K} \mathrm{k}_{1}$ indicates that the average separation is $18 \mathrm{~nm}$ and at $265 \mathrm{~K} \mathrm{k}_{1}$ is less than $0.5 \%$ $\mathrm{BZ}$, or the separation greater than $40 \mathrm{~nm}$ showing that the island density has decreased by a factor of $\sim 20$ (in excellent agreement fig. 1$)$. The oscillation period of $1 / 4(2 \pi / d)$ which indicates that the two reflecting surfaces the electrons scatter from are the island tops and the $4 \times 1$ interface, not the top of the wetting layer similar to the scattering on top of the $\beta$ $\sqrt{ } 3 x \sqrt{ } 3^{[12]}$. Otherwise the oscillation period would be $1 / 2(2 \pi / d)$.

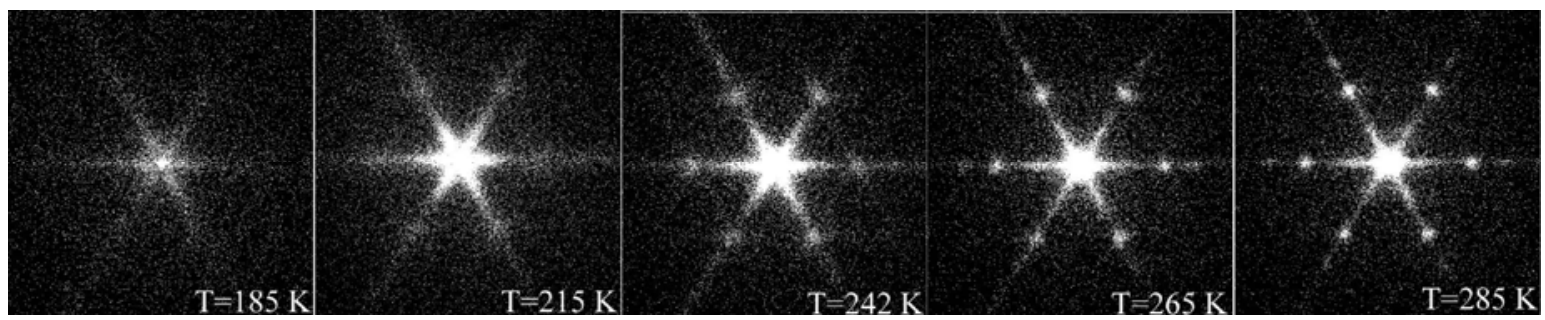

Figure 2 Diffraction patterns of $40 \%$ x $40 \%$ BZ (Brillouin Zone) the specular beam of 3ML with annealing temperature. Arms are caused by the anisotropic island shapes and corrugation spots visible at $11 \%$ and $14 \% \mathrm{BZ}$ along ([1_10]) confirm that the $\mathrm{Pb}(111)$ island is in contact with the $\operatorname{In}(4 \times 1)$ interface.

Annealing experiments on other interfaces with SPA-LEED ${ }^{[12]}$ and x-ray diffraction ${ }^{[5]}$ to $\mathrm{RT}$ have shown that as $\mathrm{Pb}$ islands are annealed, the distribution of island heights changes irreversibly from a single preferred height to a broader height distribution. As mentioned above, an important question is can the sharply peaked distribution be stable at higher temperatures? The extraordinary stability of the 4 layer islands on $\operatorname{In}(4 x 1)$ is still not understood theoretically, i.e. why the presence of In has such a drastic effect on the energetics. With STS the energy levels of the $\mathrm{Pb}$ islands on $\operatorname{In}(4 \mathrm{x} 1)$ were found to be $\sim 1$ $\mathrm{eV}^{[4,14]}$, while the calculated levels for free standing films were measured to be $2.2 \mathrm{eV} .{ }^{[15]}$ Either way, the difference in surface energy, especially when the electronic energy is summed over the subbands in the parallel $\mathrm{k}_{\|}$lateral direction, will be only a few meVs. ${ }^{[15]}$ Also, since the calculations of [15] assumed infinite lateral size of the islands, it is unclear whether the unusual shape of the islands contributes to their increased temperature stability. Regardless, the unusual temperature stability implies that it is possible for suitably prepared substrates (with specific superstructures grown on top) to enhance island stability to higher annealing temperatures. 


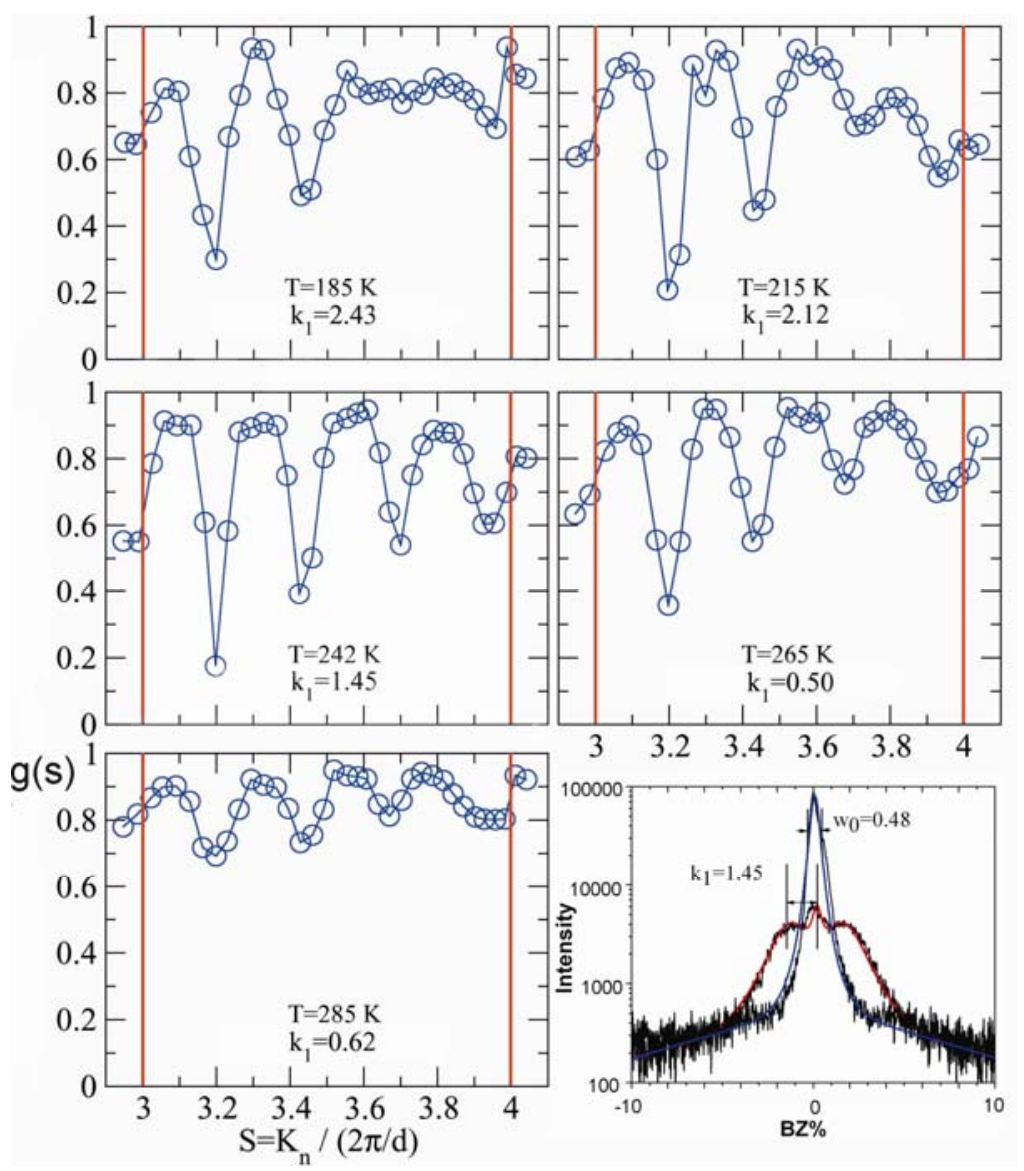

Figure $3 \mathrm{G}(\mathrm{s})$ curves taken for $3 \mathrm{ML}$ coverage on the Si(111)-In(4x1) phase with increasing temperature. The oscillation period is $1 / 4(2 \pi / d)$ which indicates that the 4 layer island height is unchanged with annealing. At the bottom right typical in phase and out of phase conditions are shown with the satellite wavevector $k_{1}$ and instrumental width $w_{0}$ marked .

In summary, we have investigated with STM and SPA-LEED the thermal stability of anisotropic $\mathrm{Pb}$ islands grown on $\operatorname{Si}(111)-\operatorname{In}(4 \mathrm{x} 1)$. Unexpectedly, the 4 layer uniform height on this interface is found to be stable to RT which is substantially higher than previously observed on other substrates and brings the grown structures closer to technological applications.

[1] Z. Zhang, Q. Niu, C.-K. Shih, Phys. Rev. Lett., 805381 (1998)

[2] K. Budde, E. Abram, V. Yeh, M.C. Tringides, Phys. Rev. B, 6110602 (2000)

[3] S.H. Chang, W.B. Su, W.B. Jian, C.S. Chang, L.J. Chen, T.T. Tsong, Phys. Rev. B, 65 245401 (2002)

[4] M. Hupalo and M. C. Tringides, Phys. Rev. B 65, 115406 (2002)

[5] P. Czoschke, H. Hong, L. Basile, and T.-C. Chiang, Phys. Rev. B 72, 075402 (2005) 
[6] Y.-F. Zhang, J.-F. Jia, T.-Z. Han, Z. Tang, Q.-T. Shen, Y. Guo, Z. Q. Qiu, and Q.-K. Xue, Phys. Rev. Lett. 95096802 (2005)

[7] D.A. Ricci, T. Miller, T.-C. Chiang, Phys. Rev. Lett., 95266101 (2005)

[8] Y. Jia, B. Wu, H. H. Weitering, and Z. Zhang, Phys. Rev. B 74, 035433 (2006)

[9] S. Stepanovskyy, V. Yeh, M. Hupalo, M.C. Tringides, Surf. Sci., 515187 (2002)

[10] M. Hupalo and M.C. Tringides, Phys. Rev. B, 73041405 (2006)

[11] D. Thein, F.J. Meyer zu Heringdorf, P. Kury, M. Horn-von Hoegen, Anal. Bioanal. Chem. 379588 (2004)

[12] M. Hupalo, S. Kremmer, V. Yeh, L. Berbil-Bautista, E. Abram and M. C. Tringides, Surf. Sci., 493526 (2001)

[13] M. Hupalo, V. Yeh, T.L.Chan C. Z. Wang, K.M.Ho and M.C.Tringides Phys. Rev. B 71 193408 (2005)

[14] A. Mans, J. H. Dil, A. R. H. F. Ettema, and H. H. Weitering, Phys. Rev. B 66195410 (2002)

[15] C.M. Wei and M.Y. Chou, Phys. Rev. B, 66233408 (2002) 


\title{
CHAPTER 4: CRYSTALLOGRAPHIC PHASE TRANSITION AND ISLAND HEIGHT SELECTION IN IN/SI(111) GROWTH
}

A paper published in Phys. Rev. B., 77, 233302 (2008)

\author{
J. Chen ${ }^{1}$, M. Hupalo ${ }^{2}$, M. Ji ${ }^{3}$, C. Z. Wang ${ }^{3}$, K.M. Ho ${ }^{3}$ and M. C. Tringides ${ }^{4}$
}

\begin{abstract}
In/Si(111) has been studied with SPA-LEED, STM and first-principles total energy calculations to identify the growth morphology at low temperatures. Of the different substrate interfaces used, only In growth on $\mathrm{Si}(111)-\mathrm{Pb}-\alpha-\sqrt{3} \mathrm{x} \sqrt{3}$ has resulted in uniform height fcc (111) 4-layer islands. A transition to the bulk bct (110) oriented islands is favored at higher temperatures $\mathrm{T}>250 \mathrm{~K}$ and/or larger coverages $\theta>5 \mathrm{ML}$. These results suggest two stabilizing effects for the preferred morphologies, i.e. QSE and orientation dependent surface and interface energies and is supported from first principles calculations.
\end{abstract}

As nanostructures become smaller in size deviations from macroscopic bulk behavior should be expected. The discreteness in their structure and the increasing importance of low symmetry atoms (i.e. surface atoms in 3-d structures, perimeter atoms in 2-d structures etc.) can result in dramatic differences from the structure of macroscopic crystals. A rich and still unexplored realm of potentially technologically relevant metastable phases can be observed as the dimensions of the structures are reduced ${ }^{[1-5]}$.

Novel physical phenomena in reduced dimensions has been realized under a range of conditions for nanostructures smaller than some minimum size. For example kinetic barriers that control sintering (and therefore catalytic activity) in $\mathrm{Pb}$ nanoparticles adsorbed on $\mathrm{MgO}$ [1] and 2-d Xe adatom or vacancy cluster diffusion on $\operatorname{Pt}(111)^{[2]}$ were found to decrease with size, because they are determined by atoms of the lowest coordination and not the average curvature of the structure. For metallic islands with heights comparable to the wavelength of the confined electrons $\lambda F$ the island electronic structure is controlled by Quantum Size Effects (QSE) ${ }^{[6-12]}$. More importantly the confined electron energy can vary as function of height so preferred island heights were observed. In other systems novel crystallographic 
phases ${ }^{[13]}$ have been observed in nanostructures, different from the bulk phases like the fcc $\mathrm{Fe}$ grown on $\mathrm{Cu}$ substrate ${ }^{[13]}$. During the room temperature growth of $\mathrm{Bi}$ on $\mathrm{Si}(111)-7 \mathrm{x} 7$, first islands of a pseudocubic (012) phase grow at low coverage which convert to the hexagonal bulk structure (001) phase ${ }^{[14]}$.

In these systems ${ }^{[1-14]}$ the deviation from bulk behavior on the nanoscale was only of one physical parameter (either of the kinetic barriers so the speed of the process is accelerated ${ }^{[1-5]}$ or of the grown island height ${ }^{[6-12]}$ or of the crystallographic phase ${ }^{[13,14]}$ ). It would be interesting to find a single nanoscale system where more than one bulk deviation is seen and are controllable with easy "experimental" knobs. In this paper we report that In growth on the $P b-\alpha-\sqrt{3} \mathrm{x} \sqrt{3}$ is such a system where several deviations from the bulk are tunable: 4-layer uniform fcc(111) height islands due to QSE for coverages less than some critical coverage, the novel fcc(111) crystallographic phase which transforms into bulk bct(110) with increasing temperature or coverage; and enhanced diffusion on this particular $\mathrm{Pb}-\alpha-\sqrt{3} \mathrm{x} \sqrt{3}$ interface so mass transport is unusually fast that large islands form at temperatures as low as $150 \mathrm{~K}$. The bet islands prefer to grow in height and can reach multiple heights (i.e. they can easily become many times the deposited amount). Since initially fcc(111) islands are present and they should be converted to larger bct(110) islands, a large number of mixed metastable islands is also observed before all islands convert to the bct structure. It is remarkable that both the uniform height uniformity, the transition from fcc(111) to bct(110) and fast kinetics well below room temperature are only observed when growth is on the $\mathrm{Si}(111)-\mathrm{Pb}-\alpha \sqrt{3} \mathrm{x} \sqrt{3}$ phase (and not on other $\mathrm{Si}(111)$ interfaces: clean $\mathrm{Si}(7 \mathrm{x} 7$ ), In $-\beta \sqrt{ } 3 x \sqrt{ } 3$, In- $\sqrt{ } 31 x \sqrt{ } 31$, In- $4 x 1)$.

These results have been examined in parallel with first-principles total energy calculations to identify the relative contribution of the surface energy, interface energy, and the QSE energy in island stability.

Experiments were performed in two separate UHV chambers. The $\mathrm{Si}(111) \mathrm{Pb}$ $\alpha \sqrt{3} \times \sqrt{3}$ phase is prepared by depositing in excess of $1.3 \mathrm{ML}$ of $\mathrm{Pb}$ on $\mathrm{Si}-(7 \times 7)$ followed by annealing to $500 \mathrm{~K}$. Fig. 1 (a) shows an area $500 \times 418 \mathrm{~nm} 2$ at $\mathrm{T}=204 \mathrm{~K}$ with $\theta=2.7 \mathrm{ML}$ of Indium grown on $\mathrm{Si}(111)-\mathrm{Pb} \alpha \sqrt{3} \times \sqrt{3}$ showing uniform height 4-layer islands. The islands have fcc(111) structure as seen visually from their shape, their heights and as confirmed with 
diffraction shown below. The corresponding histogram is shown in fig.1(b) with extraordinary sharpness of the height distribution similar to the one observed in the intriguing $\mathrm{Pb} / \mathrm{Si}(111)$ system. These results are confirmed with SPALEED diffraction measurements that record the variation of the diffracted intensity vs electron energy as the scattering condition normal to the surface changes from in-phase to out-of-phase. It is also important that this sharp height distribution of the fcc islands is observed only on this interface $\mathrm{Pb}-$ $\alpha \sqrt{ } 3 x \sqrt{3}$ and growth on other interfaces leads to broader distribution of irregularly shaped still fcc Indium islands.

However the uniform height selection competes with a new nanoscale effect as the coverage or temperature increases. Fig.2(a) shows a 200x200nm2 STM image of In islands grown on $\mathrm{Pb}-\alpha \sqrt{3} \mathrm{x} \sqrt{3}$ at $\mathrm{T}=200 \mathrm{~K}$ with $\theta=2.5 \mathrm{ML}$. Within this area a taller island is seen top left with mixed shape, i.e. its left part has tetragonal (bct phase) and its right part has triangle shape(fcc shape). The mixed islands do not have flat top but the same number of layers in the bet and fcc part of the island. This image shows the onset of a crystallographic transition that with further deposition or with annealing it converts the initial fcc(111) to normal bct(110) oriented islands as expected from the bulk Indium bct structure.

Fig.2(b) shows an area of $280 \times 280 \mathrm{~nm} 2$ with the mixed island (located now at the center left of the image) after stepwise Indium deposition with a total of $2.5 \mathrm{ML}$. It is remarkable that the mixed island has grown from $10 \mathrm{ML}$ to $28 \mathrm{ML}$. This indicates an extraordinary high and selective Indium mobility where mass transport is preferably to the bct islands. Figs. 2(c) and 2(d) show the corresponding height histogram and the surrounding fcc(111) islands grew only from 4 to 5 -layers. In fig. 2(d) more peaks are seen because islands of both orientations being present and the bct islands have no preferred height.

These results are fully confirmed with diffraction SPA-LEED. The diffraction experiments show that with increasing temperature $\mathrm{T}$ or I coverage $\theta$ the fcc spots become weaker and eventually disappear while the bct spots grow stronger. Both 2-D and 1-D scans are shown in Fig.3 for growth at $\mathrm{T}=150 \mathrm{~K}$ with electron energy $38 \mathrm{eV}$. Figs. 3(a) and 3(c) correspond to $\theta=4 \mathrm{ML}$ while Figs. $3(\mathrm{~b})$ to $\theta=8 \mathrm{ML}$ and $3(\mathrm{~d})$ to $\theta=6 \mathrm{ML}$. The red scans are for the clean $7 \times 7$ surface and are used for the wavevector calibration. The scanning directions in 
$3(\mathrm{c})$ is $[1 \overline{1} 0]$ and in $3(\mathrm{~d})$ for the black curve is 4deg off the [1 $\overline{1} 2]$ and for the red curve is $[1 ! 12]$.
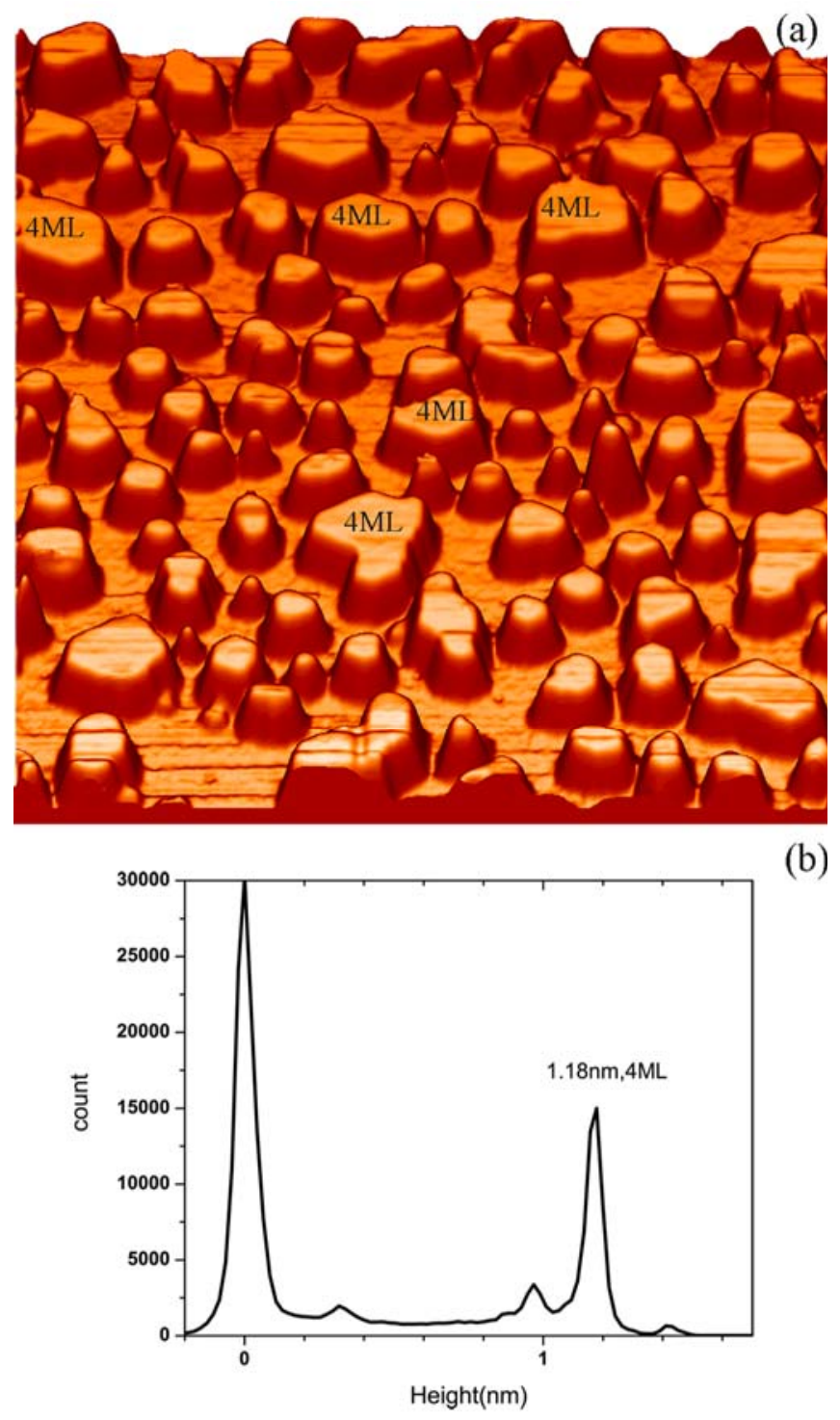

(b)

Figure 1 (a) 500x418nm ${ }^{2}$ T=204K $\theta=2.7$ ML Indium grown on $\mathrm{Si}(111)-\mathrm{Pb}-\alpha-\sqrt{3} \mathrm{x} \sqrt{3}$ showing uniform height 4-layer fce(111) islands as seen in the histogram of fig1(b).

These diffraction data give directly the fcc(111) and bct (110) planar unit cells from the spot positions: the fcc(111) spots along [ $\overline{1} 10]$ are at $115.2 \% \mathrm{BZ}$ and for the bet(110) spots

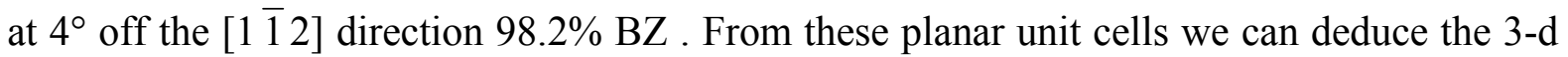
fcc unit cell (the lattice constant is $0.477 \mathrm{~nm}$ ) and the $\mathrm{c}$ axis lattice constant $\mathrm{c}=0.495 \mathrm{nmnm}$ of the bet $3-\mathrm{d}$ unit cell. (From the ratio $\mathrm{c} / \mathrm{a}=1.52$ we obtain the lattice constant in the other two directions $\mathrm{a}=0.325 \mathrm{~nm})$. These extrapolated values of the $3-\mathrm{d}$ unit cells are essentially the 
same as the bulk ones which indicates that the islands are not strained. From these values we deduce the interlayer spacing of the fcc(111) planes to be $0.274 \mathrm{~nm}$ and for the bct(110) planes $0.231 \mathrm{~nm}$. (When the STM preferred fcc heights are converted into number of mono layers the ratios are non-integers 4.2ML, because STM measures contours of electron density from the wetting layer). The area between the In islands is covered by the $\alpha-\sqrt{3} x \sqrt{3}$ phase as seen by the strong $(1 / 3,1 / 3)$ and $(2 / 3,2 / 3)$ spots, although as concluded from low coverage experiments these correspond to mixed In and $\mathrm{Pb}$ "Devil's Staircase" phases. This confirms the tendency of the In-Pb system to form a 2-D smooth alloyed phase which has even higher In mobility and can account for the extraordinary speed of building the mixed bct + fcc islands ${ }^{[15]}$. The characteristic spots at $14 \% \mathrm{BZ}$ in Fig. 2 (a) close to the (00) spot originate from the Moire pattern at the interface (8 Indium match 7 Si unit cells).

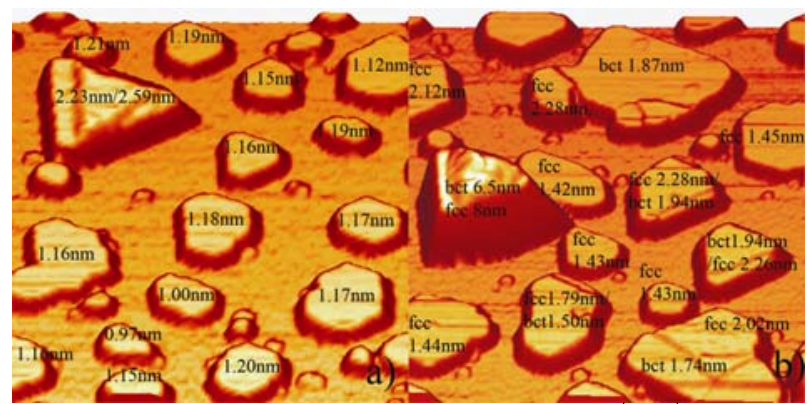

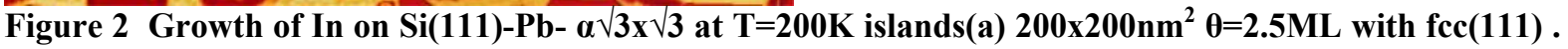
(b) $280 \times 280 \mathrm{~nm} 2 \theta=5 \mathrm{ML}$ with fcc(111), bct(110) and mixed islands. The mixed island grew by $18 \mathrm{ML}$ although only $2.5 \mathrm{ML}$ has been deposited.

It is well known that the bct crystal structure is the ground state structure of In. Previous first-principles calculations have shown that the energy of bulk fcc In is only slightly higher than bulk bct, by approximately $2 \mathrm{meV} /$ atom $^{[16]}$. They are sufficiently close for the transition between the two structures to be possible especially for low dimensional islands at smaller thickness.

In order to verify this competition, first-principles calculations were performed using the plan-wave pseudopotential VASP code ${ }^{[17]}$. Interactions between the core and valence electrons are described by the projected augmented wave (PAW) potential ${ }^{[18]}$. Exchange correlation energy is described by the generalized conjugate gradient approximation (GGA) using the form proposed by Perdew-Burke-Ernzerhof (PBE) ${ }^{[19]}$. The energy cutoff for the plane wave basis was $350 \mathrm{eV}$. The optimized lattice constants from calculations are $0.331 \mathrm{~nm} / 0.503 \mathrm{~nm}(\mathrm{i} / \mathrm{e} . \mathrm{a} / \mathrm{c})$ for the bct bulk structure and $0.48 \mathrm{~nm}$ for the fcc structure, which 
are in good agreement with the experimentally measured ones $0.325 \mathrm{~nm} / 0.495 \mathrm{~nm}$ and $0.471 \mathrm{~nm}$ respectively. The calculated cohesive energies for the two crystalline structures are -2.7208 and $-2.7223 \mathrm{eV}$ per atom respectively, consistent with the previous results ${ }^{[16]}$.
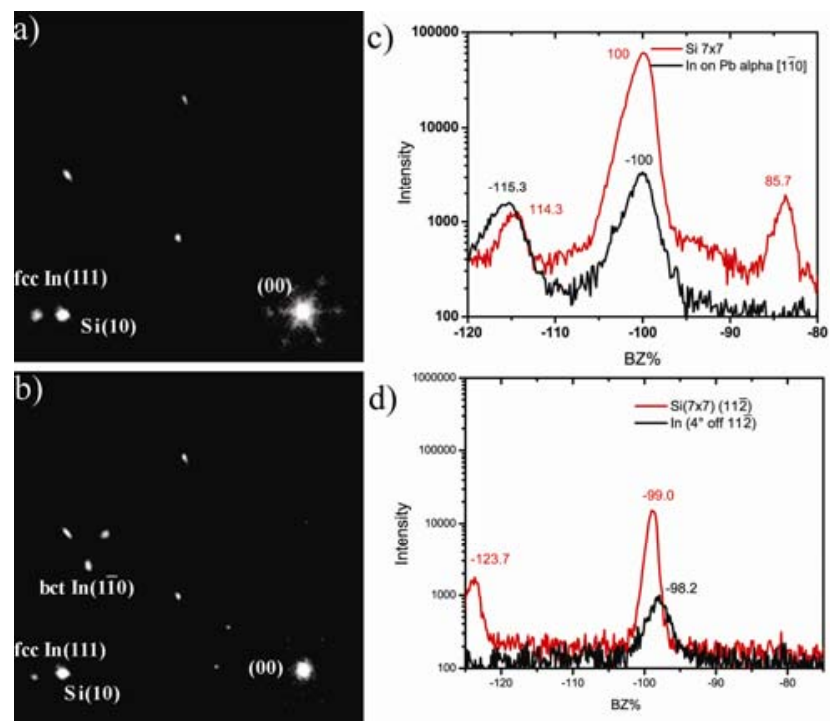

Figure 13 2-d diffraction patterns and 1-d scans for In growth on $\operatorname{Si}(111)-\mathrm{Pb}-\alpha \sqrt{3} \times \sqrt{3} \mathrm{~T}=150 \mathrm{~K}$ with electron energy $38 \mathrm{eV}$. Figs. 2(a) shows fec(111) growth with $\theta=4 \mathrm{ML}$ and fig.2(b) mixed bet(110) and fce(111) growth with $\theta=8 \mathrm{ML}$. Fig.3(c) shows 1-d scans along [ $\overline{1} 10]$ and fig. 3(d) scans for $\theta=6 \mathrm{ML} 4^{\circ}$ off

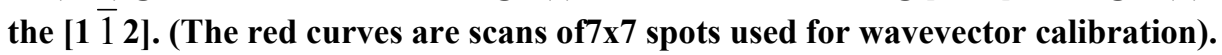

The thin film calculations were performed for the fcc In terminated by the (111) plane and for the bct In terminated by the (110) plane as seen in the experiment. The unit cell used in the calculation is $6.2 \mathrm{~nm}$ in $\mathrm{z}$-direction including the vacuum region normal to the surface and periodic boundary conditions. The thickness of the films varies from 2 to 19 layers and 20x20x1 Monkhorst-Pack k-point mesh was used for Brillouin zone sampling. In all cases the film was relaxed until the forces were less than $0.1 \mathrm{eV} / \mathrm{nm}$.

The surface energies Es of the two structures as a function of thickness are shown in Fig. 3(a) for the free standing slab calculations. The surface energy of the fcc(111) is lower than the one of the bct(110), but oscillations are seen with film thickness. These oscillations can be attributed to the Quantum Size Effect (QSE) with the In fcc(111) film being more stable at 4, 7, and 10ML, and the bct(110) film more stable at 6 and 16ML.

We note that according to the energy differences between the fcc and bct structures in the present calculations, the fcc to bct transition would be at over $100 \mathrm{ML}$ thickness which is much larger than the observed one. This difference would be due to the fact that free standing films are used and not the real $P b-\alpha-\sqrt{3} x \sqrt{3}$ substrate. The interface energy is expected to 
differ for these two interfaces and will affect the film stability. The total energy of the film can be written as $E(n)=E_{s}{ }^{0}+E_{i}+n E_{b}+E_{Q S E}(n)$ where $n$ is the number of layers, $E_{s}{ }^{0}$ is the surface energy in the limit of large thickness (so that the QSE is negligible), $E_{i}$ is the interface energy (between the metal film and substrate), $E_{b}$ is the bulk energy (per layer) and the $E_{\mathrm{QSE}}(\mathrm{n})$ is the energy as the function of film thickness due to QSE. Then the relative energy between the two film structures is $\Delta \mathrm{E}_{\mathrm{bct-fcc}}(\mathrm{n})=\Delta \mathrm{E}_{\mathrm{s}}{ }^{0}+\Delta \mathrm{E}_{\mathrm{i}}+\mathrm{n} \Delta \mathrm{E}_{\mathrm{b}}+\Delta \mathrm{E}_{\mathrm{QSE}}(\mathrm{n})$. Using the surface energy of the free standing film from Fig. 3 (a) and noting that $\mathrm{E}_{\mathrm{s}}=\mathrm{E}_{\mathrm{s}}{ }^{0}+\left(\mathrm{E}_{\mathrm{QSE}}\right) / 2$, $\Delta \mathrm{E}_{\mathrm{s}}{ }^{0}$ and $\Delta \mathrm{E}_{\mathrm{QSE}}(\mathrm{n})$ can be estimated. Calculation of the interfacial energy difference between the two film structures is not feasible because the structure of the interface is not known. Since the transition from fcc to bct occurs earlier than the free standing slab prediction, this suggests that the interface energy of the bct film is lower than that of the fcc film on $\mathrm{Pb}-\alpha-$ $\sqrt{3} x \sqrt{3}$ substrate. If we assume the interface energy difference between the two structures is $\Delta \mathrm{E}_{\mathrm{i}}=-0.16 \mathrm{eV}$, then the relative energy of the two structures $\Delta \mathrm{E}_{\text {bct-fcc }}(\mathrm{n})$ as a function of the film thickness $\mathrm{n}$ can be plotted in Fig. 3(b). The plot shows that there is strong energetic competition for the growth of fcc and bct thin films below 12 layers most likely due to the QSE. The bct structure is more favorable above 12 layers.

These calculations are indicative of the ease of transformation of the crystallographic transition with $\mathrm{T}$ or $\theta$ and the existence of mixed crystals. However given that in the experiment initially the fcc islands form, any transformation to bct(110) islands should not only be controlled by energy differences but also by the kinetic barriers to be overcome. At the low temperatures $<200 \mathrm{~K}$ these barriers can account for the extraordinary stability of the 4 -layer fcc islands below some minimum coverage $\sim 4 \mathrm{ML}$. (The calculation compares two ideal crystal structures and their $\mathrm{T}=0$ thermodynamic difference).

Earlier results in the literature have indicated partial information on the crystallographic transition. In Ref. ${ }^{[20]}$ Indium nanoparticles within Ar gas atmosphere at $300 \mathrm{~K}$ were found with X-ray scattering to transform from bct to fcc within the range 4-6nm. In Ref. ${ }^{[21]}$ TEM studies under UHV conditions of Indium nanoparticles deposited at room temperature (RT) on carbon films undergo an fcc to bct transition with crossover size at $6 \mathrm{~nm}$. 

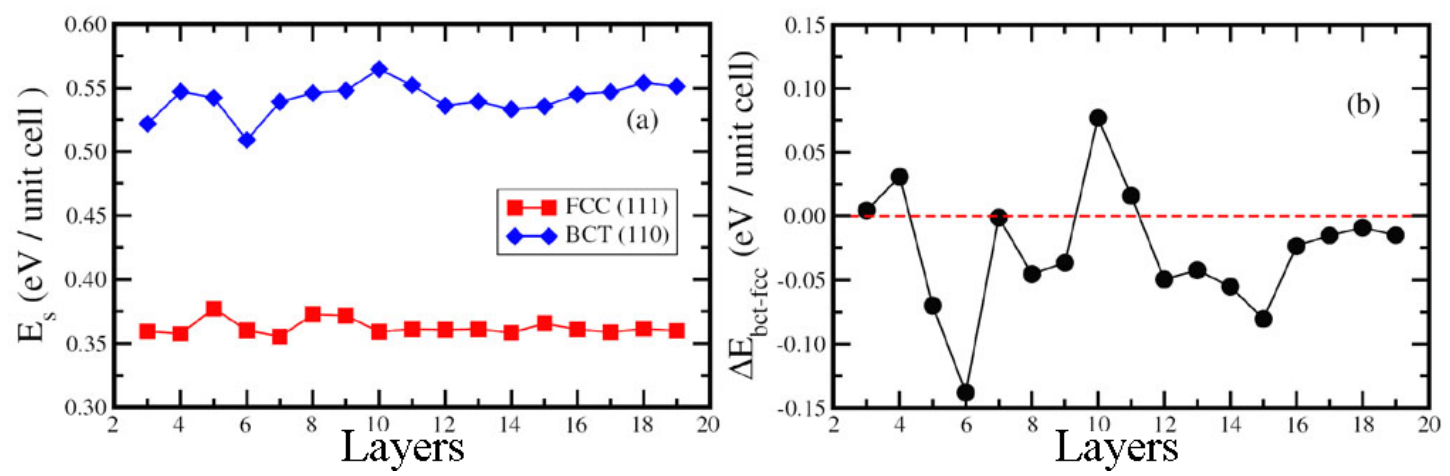

Figure 4 (a) Surface energies Es(n) of the Indium fec (111) and bct(110) films as a function of thickness n for free standing slab calculations; (b) The difference in energy between the bct(110) and fcc(111) films as a function of $n$ assuming the interface energy difference $\Delta \mathrm{Ei}=\mathbf{- 0 . 1 6 \mathrm { eV }}$. Within a narrow coverage range at $\sim 8 \mathrm{ML}$ both the bet(110) and fcc(111) films become of preferred energy.

Epitaxial growth of Indium on $\operatorname{Si}(111)-7 \times 7$ at RT was carried out with LEEM $^{[22]}$ and

STM ${ }^{[23]}$ showing In(111) islands. At temperatures higher than RT ( 440K)large islands of linear size more than $1 \mu \mathrm{m}$, with rectangular cross-section and bct(110) orientation consistent with the temperature trend of the current experiments ${ }^{[22]}$. The island density is extremely low, i.e. less than 2 islands $/ 100 \mu \mathrm{m}^{2}$ which indicates the extraordinary mobility of the Indium on the wetting layer. The In islands were also studied with STS ${ }^{[24]}$ and ARPES ${ }^{[25]}$ to conclude that electron localization effects originating from electron correlations are important.

In summary this work on $\mathrm{In} / \mathrm{Si}(111)$ shows that other metals besides $\mathrm{Pb} / \mathrm{Si}(111)$ can have similar sharp island height uniformity. The optimal interface is $\operatorname{Si}(111)-\mathrm{Pb}-\alpha-\sqrt{3} x \sqrt{3})$ and the preferred height is 4-layer. In addition an allotropic fec to bet transition is observed with a well-defined kinetic pathway how each crystal structure is attained with temperature and/or coverage. These results suggest two effects to be responsible, i.e. QSE stabilize height and surface energy stabilize the crystal structure. These conclusions are supported quantitatively with first-principles calculations. This work clearly shows the possibility of manipulating not only the island height but the crystal structure itself and with fast kinetics at such low temperatures.

\section{Acknowledgements}

Work at the Ames Laboratory was supported by the Department of Energy-Basic Sciences under Contract DE-AC02-07CH11358. This research used resources of the National Energy Research Scientific Computing Center, which is supported by the Office of Science of the U.S. Department of Energy under Contract No. DE-AC02-05CH11231. 
References:

1. C. T. Campbell, et al. Science 298, 811 (2002).

2. D. S. Sholl and R. T. Skodje Phys. Rev. Let. 75, 3158 ( 1995).

3. D.J. Liu, J.W. Evans Phys. Rev. B 66, 165407 (2002).

4. F. Nita and A. Pimpinelli Surf. Sci. 55131 (2004).

5. Z. Kuntova et al Phys. Rev. B 71, 125415 (2005)

6. K. Budde et al. Phys. Rev. RC B 61, 10602 (2000).

7. M. Hupalo et al. Phys Rev. B 64, 155307 (2001).

8. W. B. Jian et al. Phys. Rev. Lett. 90, 196603 (2003)

9. H.Hong, et al. Phys. Rev. Lett 90, 076104 (2003)

10. A. Mans, et al. Phys. Rev. B 66, 195410 (2002).

11. C. M. Wei and M. Y. Chou Phys. Rev. B 66, 233408 (2002)

12. Z.Y. Zhang, et al. Phys. Rev. Lett. 80, 5381 (1998).

13. A. Kirilyuk et al Phys. Rev. B 541050 (1996); A. Biedermann et al Phys. Rev. Lett. 86 464 (2001)

14. T. Nagao et al Phys. Rev.Lett. 93, 105501 (2004).

15. J. Chen, et al. ( to be published).

16. S. I. Simak, et al. Phys. Rev. Lett. 85, 142(2000)

17. G. Kresse and J. Furthmuller Phys. Rev. B 54,11169 (1996)

18. G. Kresse, and J. Joubert, Phys. Rev. B 59,1758(1999)

19. J. P. Perdew et al. Phys. Rev. Lett. 77, 3865(1996)

20. Akimichi Yokozeki and Gilbert Stein, J. Appl. Phys. 49(4) (1978)

21 Yoshifumi Oshima, et al, Surface Science 476107 (2001).

22 A, Pavloska, et al. Jou. Vac. Sci. Techn. B 20(6) 2478 (2002)

23. S. L. Surnev et al. Jou. Vac. Sci. Tech. A 13(3) 1389 ( 1995)

24. I. B. Altfeder, et al. Phys. Rev. Lett. 92, 226404 (2004)

25. J.H.Dil, et al. Phys. Rev. B 73, 161308 (2006). 


\title{
CHAPTER 5: THE EXTRAORDINARY HIGH MOBILITY OF INDIUM ATOM ON SI(111)-PB- $\alpha-\sqrt{3} \times \sqrt{ } 3$ SURFACE
}

(based on a paper to published)

\begin{abstract}
Indium islands are grown on $\mathrm{Si}(111)-\mathrm{Pb}-\alpha-\sqrt{3} \mathrm{x} \sqrt{3}$ surface and studied via scanning tunneling microcopy(STM) and spot-profile-analysis low energy electron diffraction(SPALEED). We found indium atoms have extra high mobility and they highly prefer to coarsen to huge islands than smaller islands.
\end{abstract}

Introduction

One of the unusual systems that has generated a lot of interest recently is $\mathrm{Pb} / \mathrm{Si}(111)$. ${ }^{[1,2,3]}$ Recently, experiments on the $\mathrm{Pb} / \mathrm{Si}(111)$ system has shown that not only quantum size effect(QSE) ${ }^{[4]}$ are observable in nanostructures, but also because of the variation of the electronic energy with height an extremely sharp height distribution is obtained. In the varies of $\mathrm{Pb} 2 \mathrm{D}$ phases $\mathrm{Si}(111)-\mathrm{Pb}-\alpha-\sqrt{3} \times \sqrt{3}$ phase is especially good for growing islands due to its smoothness and commensuration with silicon lattice underneath. There are multiple discoveries related to it. ${ }^{[5,6]}$ One of our previous research shows QSE effect when we grow indium islands on $\mathrm{Pb}$ alpha phase and there is an phase transition of indium between fcc and bct structure. ${ }^{[7]}$ But there is no indication of QSE based on known report about growth of indium directly on $\mathrm{Si}(111) 7 \times 7 .{ }^{[8,9]}$ Therefore the $\mathrm{Pb}$ plays some important role. The interaction between $\mathrm{Pb}$ and $\mathrm{In}$ should be important. Therefore it is interesting to study the growth of In on $\mathrm{Pb}$ substrate or $\mathrm{Pb}$ growth on In substrate. Our previous work shows that the $\mathrm{Pb}$ islands growing on top of In $4 \times 1$ phase are extraordinarily stable, they remains their shape at even $\mathrm{RT}^{[10]}$. In this work we report indium atoms show an extra high mobility on $\mathrm{Pb}$ alpha phase even in low temperature, compared with the previous work on the coarsening of $\mathrm{Pb}$ islands. ${ }^{[11,12]}$ And the islands size and structure plays an extra important role in the island growth. 


\section{Experiment}

Experiments were performed with an Omicron variable-temperature STM. The system is in a UHV chamber. The $\operatorname{Si}(111)-\mathrm{Pb}-\alpha-\sqrt{3} x \sqrt{3}$ phase is prepared by depositing $\sim 1.5 \mathrm{ML}$ of $\mathrm{Pb}$ on the clean $\mathrm{Si}(111) 7 \mathrm{x} 7$ surface and then annealing to $300^{\circ} \mathrm{C}$. Fig.1 is an $\mathrm{STM}$ image of the $\mathrm{Si}(111)-\mathrm{Pb}-\alpha-\sqrt{3} \times \sqrt{3}$ phase. There are many domains in the surface. The two bright dots are islands formed by the $\mathrm{Si}$ atoms from the surface when the $7 \mathrm{x} 7$ reconstruction is destroyed. After that, the system is cool down to $180 \mathrm{~K}$ and In was deposited on it in several steps in order to observe how In islands grow. After each deposition, STM images are taken.

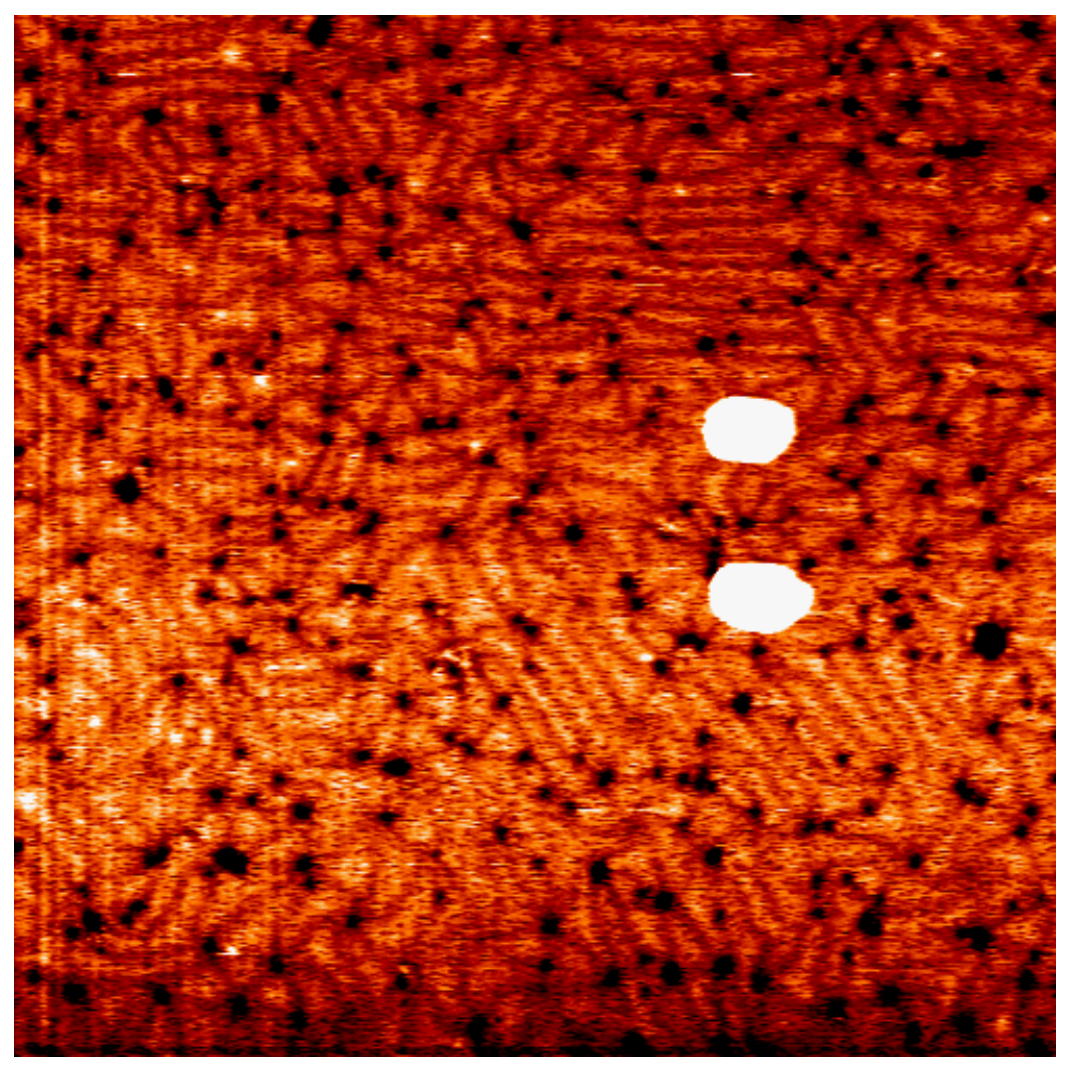

Figure $1100 \mathrm{~nm} * 100 \mathrm{~nm}$ stm image of the $\mathrm{Si}(111)-\mathrm{Pb}-\alpha-\sqrt{3} \mathrm{x} \sqrt{3}$ phase. Result and discussion

As we reported in chapter 4 , the In grows islands on top of $\mathrm{Pb} \alpha$-phase. In the beginning In islands are in face-centered cubic(FCC) structure and turn into body-centered tetragonal (BCT) structure when coverage is higher. Another discovery is that we found the big island grows much faster than the small ones. 


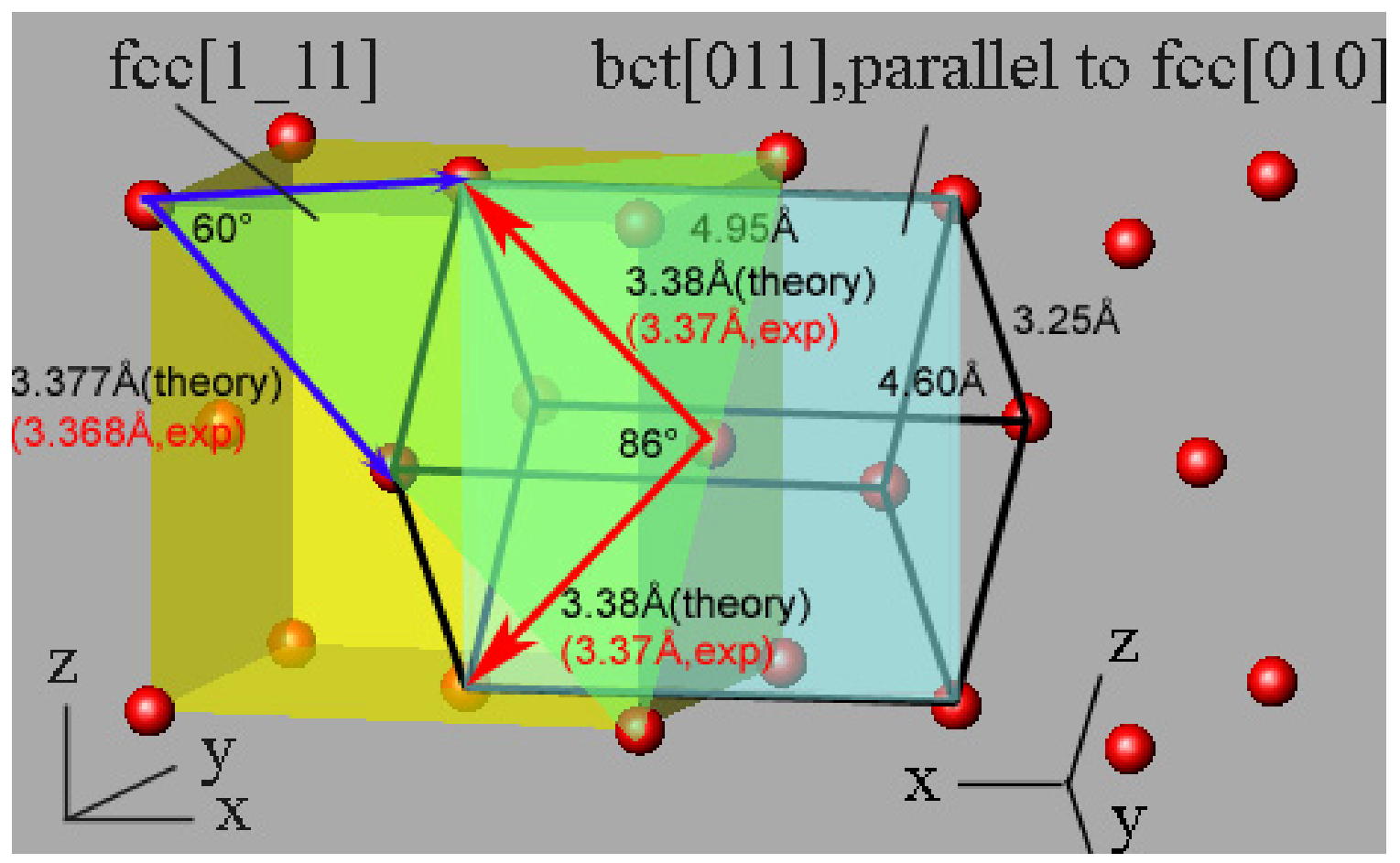

Figure 2 hard ball model for the fcc and bet planes. The red balls represents the In atoms. The unit cell and unit vectors of both structure are drawn in the figure.

Fig. 2 shows the atomic model of fcc and bet structure. The unit cell of fcc structure is shown in yellow. There is an atom in the center of each side of the cube. The fcc islands we observed is in the (111) plane (the green one in the figure). The arrows in blue give the unit vector of a primitive unit cell. The unit cell of bet structure is shown in the black frame. There is an atom in the center of each cell, so it is body-centered. We plot the unit cell of fcc and bct in the same in lattice, but actually the bct structure is slightly stretched ( 4\%) compared to fcc and its not in a real cubic structure but a tetragonal. The bet islands we observed are in its $[1 \overline{1} 0]$ plane (the light blue one) and the unit vectors of the primitive unit cell are the arrows in red.

The value of the unit vectors of fcc and bct structure can be calculated as following:

For fcc islands, it has the same structure as Si but different lattice constant. From diffraction pattern the In fcc spots is at $115 \% \mathrm{BZ}$, that is to say, the unit vector of In is 1.15 times that of $\mathrm{Si}$ in reciprocal space, so in real space the lattice constant of In fcc structure is $3.84 \AA$ (lattic constant of $\mathrm{Si}) / 1.15=3.34 \AA$. The angle between two vectors is $60^{\circ}$.

For bct islands, it has the In bulk structure (tetregonal with $\mathrm{a}=\mathrm{b}=3.2523 \AA, \mathrm{c}=4.9461 \AA$, [http://www.webelements.com/webelements/elements/text/In/xtal.html]). Therefore we can 
calculate the unit vectors from geometry. The body diagonal is $\sqrt{ }\left(\mathrm{a}^{2}+\mathrm{b}^{2}+\mathrm{c}^{2}\right)=6.755 \AA$, So the two wave vectors are $a_{1}=a_{2}=6.755 / 2=3.38 \AA$. And the angle between them is $2 * \arcsin \left(3.25^{*} \sqrt{2} / 2 / 3.38\right)=85.8^{\circ}$. Based on these unit vectors, we can calculate the unit vectors in reciprocal space: $a_{1}^{*}=2 \pi \frac{\hat{n} \times a_{1}}{a_{1} \bullet\left(a_{2} \times \hat{n}\right)}, a_{2}^{*}=2 \pi \frac{a_{2} \times \hat{n}}{a_{1} \bullet\left(a_{2} \times \hat{n}\right)}$ $\left(a_{1} *, a_{2} *\right.$ are reciprocal vectors $)$. In our case, $a_{1} *=a_{2} *=2 \pi / a_{1} \sin \theta\left(\theta=85.8^{\circ}\right.$ is the angle between $\mathrm{a}_{1}$ and $\mathrm{a}_{2}$ ). Notice that $100 \%$ Si Brillouin Zone(BZ of Si) corresponds to $\frac{4 \pi}{\sqrt{3} a_{0}}$, $a_{1} *=a_{2} *=\sqrt{ } 3 a_{0} /\left(2 a_{1} \sin \theta\right) * 100 \% B Z=98.9 \% B Z$. This value agrees with the experiment $(99 \%)$ in diffraction. (in Chapter 4)

We can also calculate the single step height of bct and fcc structure. For fcc, the islands grow on (111) plane, so the step height is simply one third of the body diagonal of the yellow cube: $4.77 / \sqrt{ } 3=2.74 \AA$. For bct, the islands grow on $\left(1 \_10\right)$ plane, so the single layer height is $3.25 / \sqrt{ } 2=2.30 \AA$. We can see the single layer height is different in the two structures and therefore in a mixed island there will be two different heights.

Fig. 3 shows the growth of In islands in different coverages at $\mathrm{T}=180 \mathrm{~K}$ at the same place. The flux rate of In deposition is $0.09 \mathrm{ML} / \mathrm{min}$ calibrated by the quartz crystal (see Chpater2). In the beginning of the observation the island \#1(the biggest) is in fcc-bct transition so it is in mixture of the two structures and therefore has two different heights, corresponding to fcc and bet, respectively. Most other islands are still fcc islands. It is obviously that the In atoms falling on to each single islands is less than the amount of the island growth. What we need notice is island \#1, which grows extraordinarily fast. It is a mixed island of fcc and bet structure. In Fig.3(b) its height is $2.23 / 2.59 \mathrm{~nm}(\mathrm{bct} / \mathrm{fcc})$, and in Fig 3(f) its height becomes $6.34 / 7.78 \mathrm{~nm}$, which is almost tripled to the height in (b). In the same time, most other islands grow only one layer in height, which is much slower although some of them (e.g. \#5 and 6) emerge and grow in size. This means most newly deposited In around islands 1 goes to it instead of other islands.

Table 1 gives the details about the growth of island \#1. The deposition time is the total time of indium deposition for each picture. The coverage is therefore the flux rate $(0.09 \mathrm{ML} / \mathrm{min})$ times the deposition time. The table also gives the time when each image is 
taken and the time interval between two depositions. Height and the size of the island are measured using the Scala software. The area and volume is then calculated by geometry. The table also gives the size of area it collects indium atoms from, and the ratio of the collection area to its own area in each of the six coverages. From Table.1 we can see Island \#1 collected an area which is much larger than its size, i.e. it collect In atoms from a very large area of the wetting layer. The wetting layer plays an important role in the growth of islands.

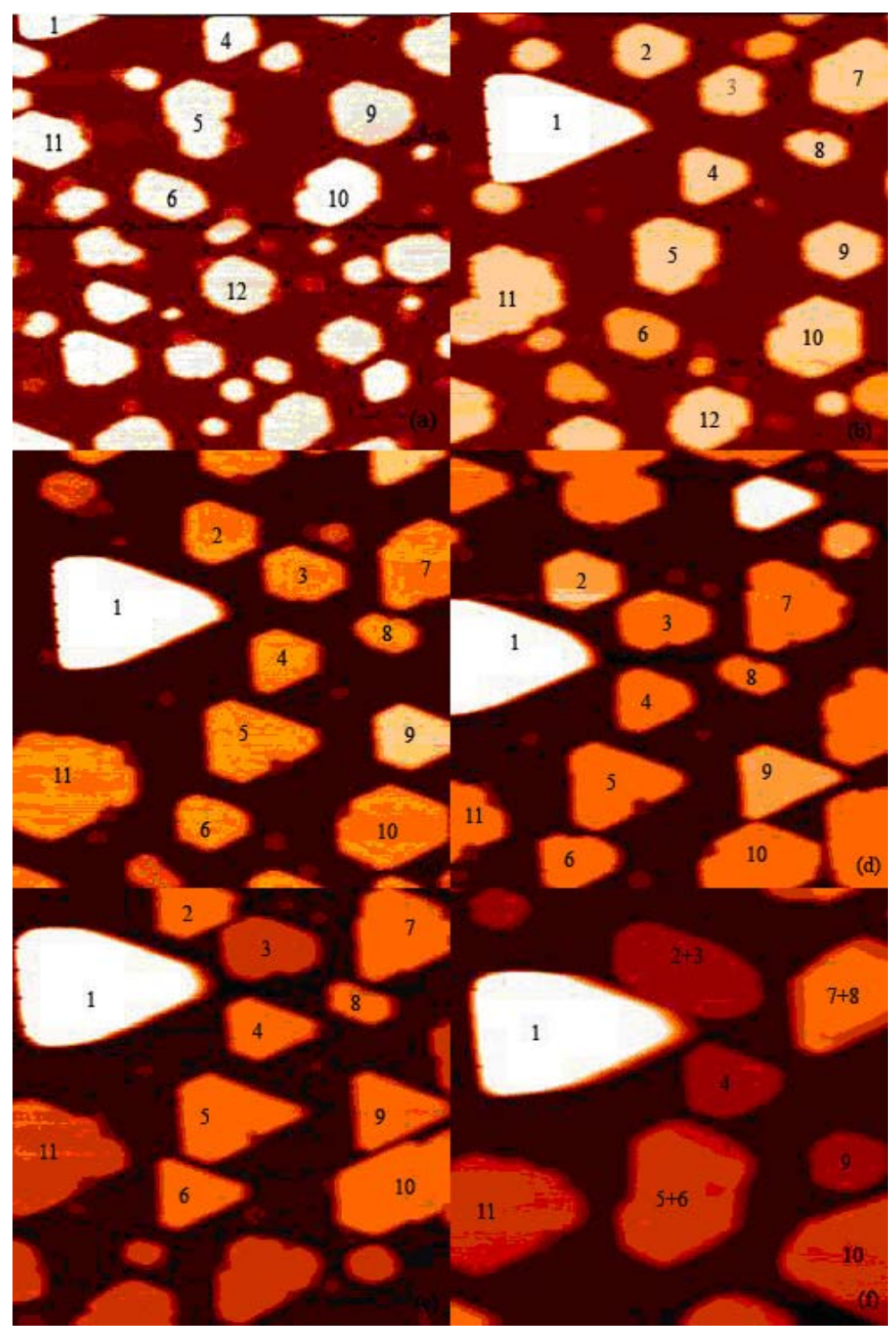

Figure 3 STM images showing the growth of islands in a specific area, all imges are $200 \mathrm{~nm} \times 200 \mathrm{~nm}$ (a) img26, 2.4ML (b) img30, 2.8ML (c) img34, 3.2ML (d) img37, 3.6ML (e)img40, 4.1ML (f) img48, 5.6ML, $\mathbf{T}=\mathbf{2 0 7 K}$. 
During the whole process, the islands \#1 grows much faster than other islands. Fig.4 shows the contrast between the island\#1 and other islands. In Table 2 we show the comparison between island \#1 and other islands. From (b) to (f), islands \#1 grows from 9ML to $28 \mathrm{ML}$, while the fcc islands around it grows only $1 \mathrm{ML}$, which means it grows about 20 times faster. Therefore most deposited In atoms must goes to it.

By subtract the volume between two images we can calculate the indium that the island collects during the time interval and then the collection area (volume difference divided by the thickness of deposited indium).

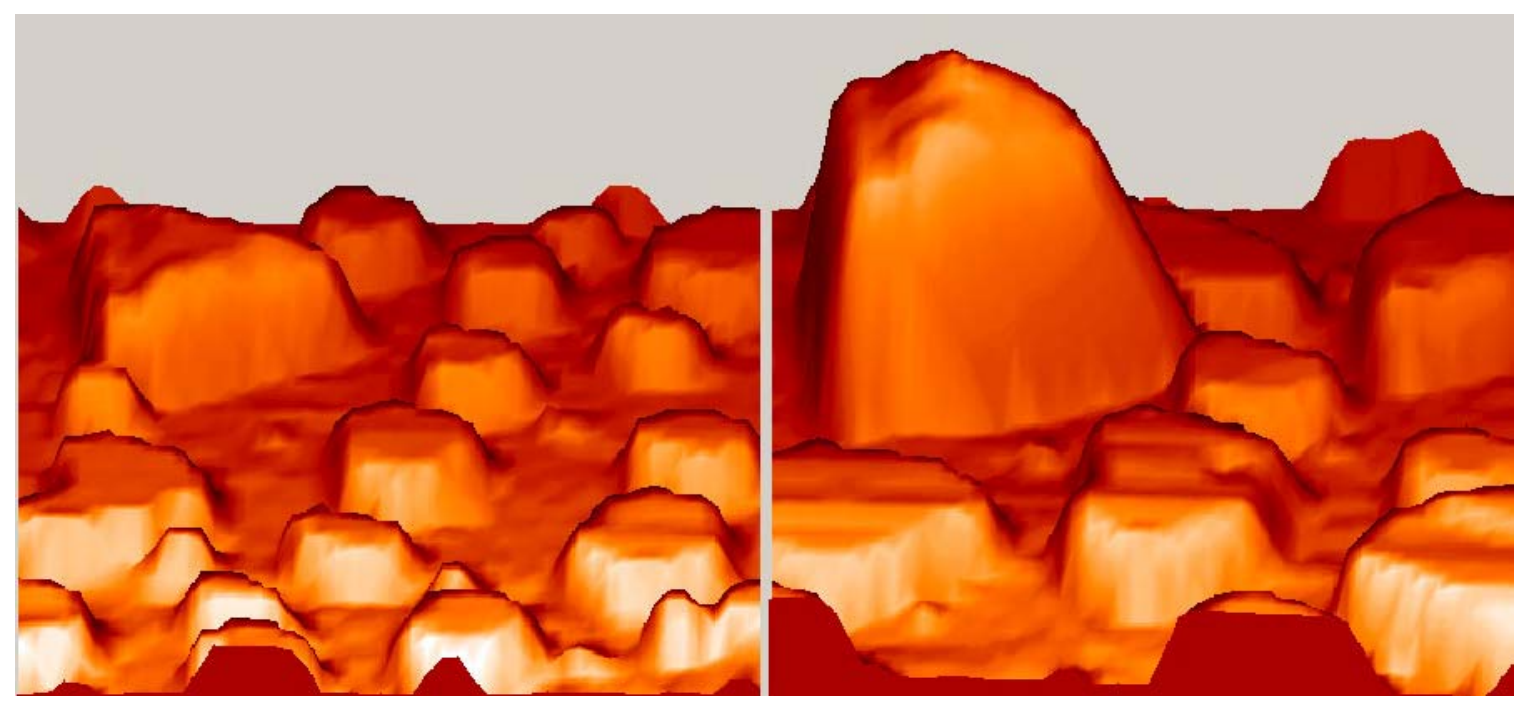

Figure 4 3D STM images showing the indium islands of the same area at coverage of (a) 2.5ML (b) 5.0ML. Image size is $200 \mathrm{~nm} \times 200 \mathrm{~nm}$. $\mathrm{T}=207 \mathrm{~K}$.

For an example, from (b) to (c), the island's area increases from $2520 \mathrm{~nm}^{2}$ to $2687 \mathrm{~nm}^{2}$, its height increases from 2.23/2.59nm (10ML) to $2.95 / 3.46 \mathrm{~nm}(13 \mathrm{ML})$ and therefore its volume increases from $5890 \mathrm{~nm}^{3}$ to $8354 \mathrm{~nm}^{3}$ (the area and volume of fcc and bct parts are calculated separately and summed up in the end due to there height difference). The increased volume is $2464 \mathrm{~nm}^{3}$. However, we put only $0.36 \mathrm{ML}$ of indium during this period, so the indium thickness is $0.36 * 0.274=0.099 \mathrm{~nm}$ and the indium atoms that directly falls on to it is $2687 \mathrm{~nm}^{2} * 0.099 \mathrm{~nm}=269 \mathrm{~nm}^{3}$. All other indium comes from the wetting layer. The collection area is $2464 / 0.099=24640 \mathrm{~nm}^{2}$. If we subtract the area of itself, then the collection area is $24640-2687=21953 \mathrm{~nm}^{2}$, which is 8 times its own area.

From above analysis, we can get to a conclusion that the indium islands collect In from area larger than its size. This means the atoms landing on wetting layer between them 
will go to the islands, too. Among them the island \#1 is very special, it collects In atoms from an area which is nearly 10 times of its size, which means atoms landing at more than 3 times of its radius will go to it instead of other nearer islands (e.g. \#2,3,...) In another word, it is like a vacuum cleaner absorbing almost all the atoms in the wetting layer. This also shows the extraordinary mobility of In atoms. The reason why In atoms so prefer the big island is still unknown.

Since the atoms falling to the wetting layer will also go to the islands, the wetting layer plays an important role. If we focus on the wetting layer we can see there are many "dots" on the wetting layer between islands. These dots are about $0.1 \mathrm{~nm}$ in height, $1.5-2 \mathrm{~nm}$ in diameter, the average distance between two dots are $\sim 2.3 \mathrm{~nm}$, their number density is about 72/ (20nm*20nm).(Fig.5) The identity of these dots are not fully known, but they are very likely formed by the indium atoms.

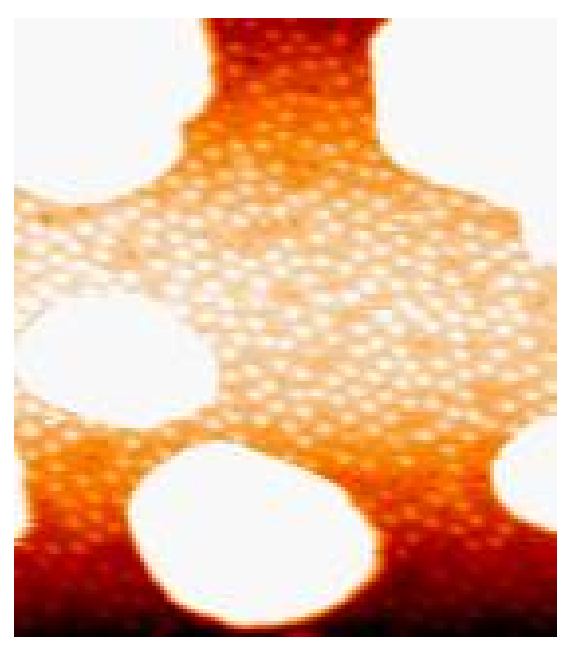

Figure 5 the "blobs" on the wetting layer. Image taken after 1.4ML of In deposited on Pb alpha phase. Image size is $28 \mathrm{~nm} * 31 \mathrm{~nm}$

The mobility of indium atoms can be compared with growth of $\mathrm{Pb}$ islands. When $\mathrm{Pb}$ islands grow on $\mathrm{Pb}$ alpha phase on $\mathrm{Si}(111)$ surface, the new layer will grow from the perimeters to the center, therefore there will be a ring ${ }^{[12]}$ in the edge in the beginning, and then the atoms filled in the inner part and to form a full additional layer. In the indium case although we made many attempts, we never found the "rings". The transfer rate from wetting layer to the island in $\mathrm{In}$ is also much larger than in $\mathrm{Pb}$. This shows that In atoms are much more mobile than $\mathrm{Pb}$ atoms. 
We can even make a rough estimation of the diffusion speed of In atoms. The time interval between two different coverages is 30 minutes. During the time, the islands \#1 absorb In atoms within the radius of $100 \mathrm{~nm}$, so the diffusion speed of In atoms is at least $100 \mathrm{~nm} / 30 \mathrm{minutes}=3 \mathrm{~nm} / \mathrm{min}$. The temperature is kept at $207 \mathrm{~K}$ during the depositions. This is the lowest speed limit for In atoms diffusion. In fact, the diffusion could finish in much less than 30minutes, so the actually diffusion speed could be much larger.

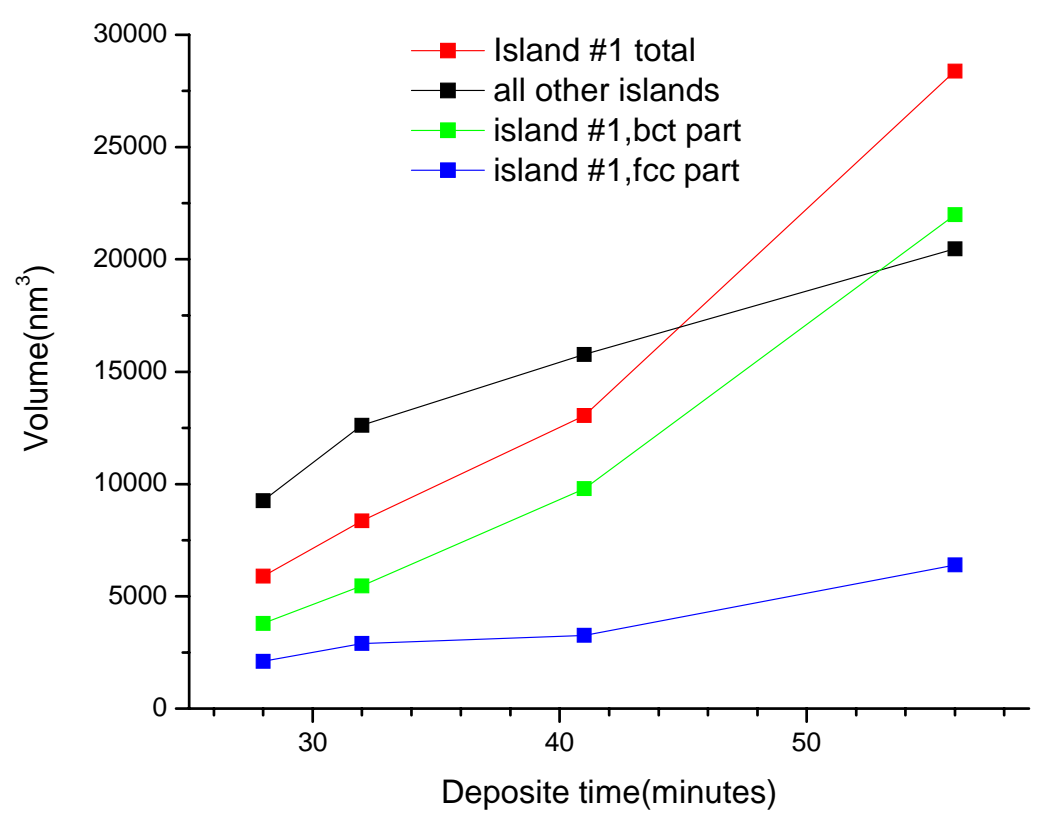

Figure 6 The growth of different parts of islands \#1 and other islands. The growth rate of the islands \#1 is much faster than other islands.

Another approach to study the properties of islands is to study the g-s curve using SPA-LEED ${ }^{[13]}$. As the electron energy varies, the normal momentum transfer component $\mathrm{Kz}$ changes as well as the phase difference between scattering from the top of the islands and the surrounding area. In certain energies, the phase difference is $0,2 \pi, 4 \pi, \ldots$ For these conditions the $\left(\begin{array}{ll}0 & 0\end{array}\right)$ beam is at a maximum and has instrumentally limited full-width-at-half-maximum (FWHM). At energies with phase difference $\pi, 3 \pi, 5 \pi \ldots$ i.e. the out-of-phase conditions there is destructive interference between the top of the islands and the surrounding region. The $\left(\begin{array}{ll}0 & 0\end{array}\right)$ profiles for out-of-phase conditions have minimum intensity and larger FWHMs. At the in-between energies, the profiles can be decomposed into a narrow and a broad 
component. The ratio $\mathrm{g}(\mathrm{s})$ of the narrow component to the total area oscillates as a function of the scaled normal component momentum transfer $\mathrm{s}=(\mathrm{Kz} /(2 \pi / \mathrm{d}))($ where $\mathrm{d}=0.286 \mathrm{~nm}$ is the single step height for $\mathrm{Pb}\left(\begin{array}{lll}1 & 1 & 1\end{array}\right)$ and $0.274 \mathrm{~nm}$ for the sing stop for $\operatorname{In}\left(\begin{array}{ll}111 \\ )\end{array}\right)$ fcc). According to the period of the $\mathrm{g}(\mathrm{s})$ curve we can learn the magic height, or the number of layers which is the dominant height of the islands. This method is proved useful in studying $\mathrm{Pb}$ islands but in indium case it is not successful because there is no obviously out-of-phase condition. This indicates that the indium islands are much mobile and the islands grow so fast that the distance between indium islands in short time exceeds the instrument' limit. But in this experiment the growth of this island is even more amazing.

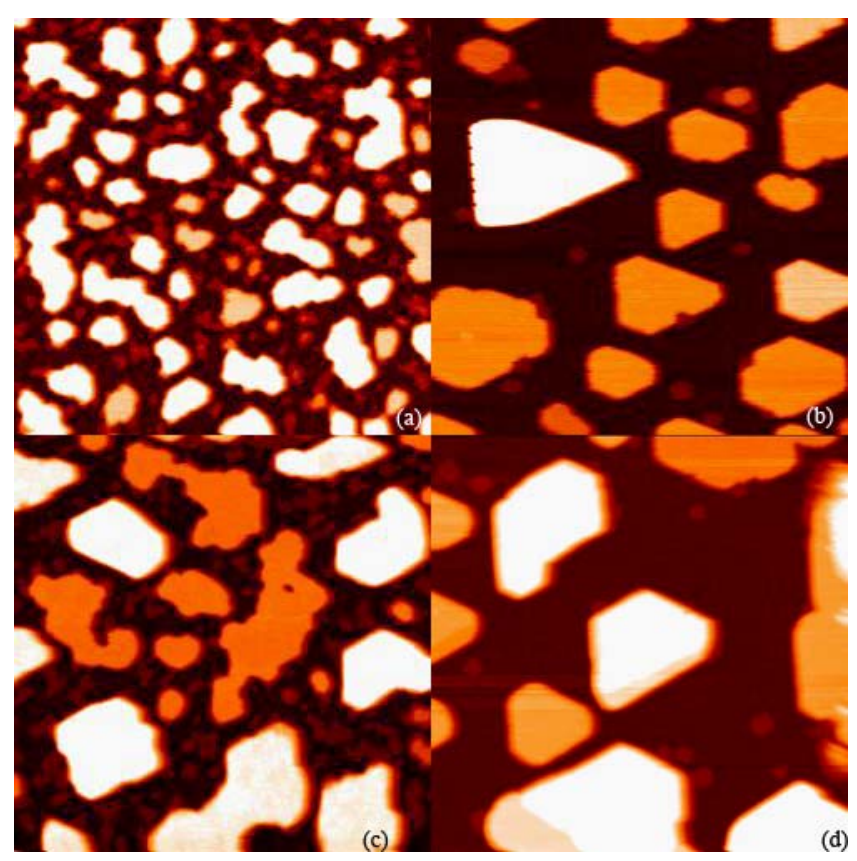

Figure 14 comparison of $\mathrm{In}$ island on $7 \times 7$ and $\mathrm{Pb}$ alpha phase (a) 34min on $7 \times 7$ (b)32 min on alpha (c) $50 \mathrm{~min}$ on $7 \times 7$ (d) $48 \mathrm{~min}$ on alpha The flux rate is the same $(0.09 \mathrm{ML} / \mathrm{min})$, the coverage is $3.0 \mathrm{ML}$, $2.9 \mathrm{ML}$, 4.5ML, 4.3ML, respectively.

The experiment also shows that bct parts are more easily to grow compared with fcc part. As we have found, the growth rate of island \#1 are much bigger than other islands. In fig.6 we give the volume of the fcc, bct part of island \#1 and other islands. We can clearly see that it grows by six times while other islands grow by only 2 times. That is to say, it grows much faster than other islands. What's more, its bct part( the green line) grows much faster than its fcc part( the blue line). 
We can also compare the difference when we deposit indium on other substrate, e.g. directly on $7 x 7$. Fig. 7 shows the islands growing on $\mathrm{Pb}$ alpha phase and directly on $\mathrm{Si} 7 \mathrm{x} 7$ surface. The islands on $7 \times 7$ surface also have preferred height, 4-layer, which is the same as the fcc islands height on $\mathrm{Pb}$ alpha phase. However, the shape of the islands is quite irregular compared with the islands on $\mathrm{Pb}$ alpha phase. They usually don't have straight side and regular shapes like triangle or hexagon. This comparison shows that the $\mathrm{Pb}-\alpha$ phase is a very special phase for the growth of In islands.

\section{Conclusion}

In atoms are more mobile than $\mathrm{Pb}$ atoms when they nucleate on the $\mathrm{Si}(111)-\mathrm{Pb}-\alpha-$ $\sqrt{3} x \sqrt{3}$ surface, especially when the bct islands have formed. They are so mobile that the STM system can't catch of figure of ring on top of In islands like Pb. The STM figures shows bet parts of In islands prefer more to grow in height and have more ability to absorb In atoms nearby, which results a contrast of growth speed between islands in different sizes. We observed that the bct islands' ability to attract In atoms in the wetting layer is much stronger than fcc islands(in fig.3, 20 times). The extraordinary ability to collecting atoms from wetting layer is very impressive and further studies are needed to fully understand the reason.

\section{References}

[1] L. Seehofer, G. Falkenberg, D. Daboul, and R. L. Johnson, Phys. Rev. B 51, 13503 (1995).

[2] M. Hupalo, J. Schmalian, and M. C. Tringides Physical Review Letters 90216106 (2003)

[3] H. Y. Lin, Y. P. Chiu, L. W. Huang, Y. W. Chen, T. Y. Fu, C. S. Chang, and Tien T.

Tsong Physical Review Letters 94136101 (2005)

[4] C. M. Wei and M. Y. Chou, Phys. Rev. B 66, 233408 (2002) (QSE)

[5] M. Hupalo, V. Yeh, T. L. Chan, C. Z. Wang, K. M. Ho, and M. C. Tringides, Phys. Rev. $B$ 71, 193408 (2005)

[6] W. B. Jian, W. B. Su, C. S. Chang, and T. T. Tsong, Phys. Rev. Lett. 90, 196603 (2003).

[7] J. Chen , M. Hupalo, M. Ji, C. Z. Wang, K.M. Ho and M. C. Tringides, Phys. Rev. B 77, 233302 (2008) 
[8] Pavlovska, E. Bauer and M. Giessen, J. Vac. Sci. Technol. B 20, 2478 (2002)

[9] J. Kraft, M. G. Ramsey, and F. P. Netzer, Phys. Rev. B 55, 5384 (1997)

[10] M. Yakes, J. Chen, M. Hupalo, M. C. Tringides Applied Physics Letters 90163117 (2007)

[11] Z. Kuntova, M. Hupalo, Z. Chvoj, and M. C. Tringides Phys. Rev. B 75, 205436 (2007)

[12] M. Hupalo and M. C. Tringides, Phys. Rev. B 75, 235443 (2007)

[13] S. Stepanovsky, M. Yakes, V. Yeh, M. Hupalo, M.C. Tringides, Surface Science 600, $1417(2006)$

Table 1 growth of Island \#1

\begin{tabular}{|c|c|c|c|c|c|c|c|}
\hline & $\begin{array}{c}\text { deposition } \\
\text { time } \\
\text { (minutes) }\end{array}$ & $\begin{array}{c}\text { Coverage } \\
\text { (ML) }\end{array}$ & time & $\begin{array}{c}\text { time } \\
\text { interval }\end{array}$ & $\begin{array}{l}\text { Height } \\
(\mathrm{nm})\end{array}$ & $\begin{array}{l}\text { Correspond } \\
\text { layer height } \\
(\mathrm{ML}, \mathrm{bct} / \mathrm{fcc})\end{array}$ & $\begin{array}{c}\text { fcc area } \\
\left(\mathrm{nm}^{2}\right)\end{array}$ \\
\hline $\mathrm{a}$ & 24 & 2.2 & $15: 48$ & & $\mathrm{n} / \mathrm{a}$ & $\mathrm{n} / \mathrm{a}$ & $\mathrm{n} / \mathrm{a}$ \\
\hline $\mathrm{b}$ & 28 & 2.5 & 16:09 & $0: 21$ & $2.23 / 2.59$ & $9.7 / 9.45$ & 812.1 \\
\hline $\mathrm{c}$ & 32 & 2.9 & $16: 30$ & $0: 21$ & $2.95 / 3.46$ & $12.8 / 12.6$ & 838.1 \\
\hline d & 36 & 3.2 & $16: 51$ & $0: 21$ & $3.24 / 3.74$ & $14.1 / 13.6$ & 765.8 \\
\hline e & 41 & 3.7 & $17: 13$ & $0: 22$ & $3.67 / 4.54$ & $16.0 / 16.6$ & 717.9 \\
\hline \multirow[t]{2}{*}{$\mathrm{f}$} & 56 & 5.0 & $17: 59$ & $0: 46$ & $6.34 / 7.78$ & & 821.9 \\
\hline & fcc volume & $\begin{array}{l}\text { bct area } \\
\left(\mathrm{nm}^{2}\right)\end{array}$ & $\begin{array}{c}\text { bct } \\
\text { volume } \\
\left(\mathrm{nm}^{3}\right)\end{array}$ & $\begin{array}{c}\text { total area } \\
\left(\mathrm{nm}^{2}\right)\end{array}$ & $\begin{array}{c}\text { total } \\
\text { volume } \\
\left(\mathrm{nm}^{3}\right)\end{array}$ & $\begin{array}{c}\text { require area } \\
\text { to } \\
\text { accumulated } \\
\text { indium for } \\
\text { island \#1 } \\
\left(\mathrm{nm}^{2}\right)\end{array}$ & $\begin{array}{l}\text { ratio to } \\
\text { its size }\end{array}$ \\
\hline $\mathrm{a}$ & $\mathrm{n} / \mathrm{a}$ & $\mathrm{n} / \mathrm{a}$ & $\mathrm{n} / \mathrm{a}$ & $\mathrm{n} / \mathrm{a}$ & $\mathrm{n} / \mathrm{a}$ & & \\
\hline $\mathrm{b}$ & 2103 & 1698 & 3787 & 2510 & 5890 & 10162 & 4.05 \\
\hline $\mathrm{c}$ & 2900 & 1849 & 5455 & 2687 & 8354 & 24640 & 9.17 \\
\hline d & 2864 & $\mathrm{n} / \mathrm{a}$ & $\mathrm{n} / \mathrm{a}$ & $\mathrm{n} / \mathrm{a}$ & $\mathrm{n} / \mathrm{a}$ & $\mathrm{n} / \mathrm{a}$ & $\mathrm{n} / \mathrm{a}$ \\
\hline $\mathrm{e}$ & 3259 & 2665 & 9781 & 3383 & 13040 & 25151 & 7.43 \\
\hline $\mathrm{f}$ & 6394 & 2520 & 15795 & 3342 & 22369 & 31201 & 9.34 \\
\hline
\end{tabular}


Table 2 Comparison between Island \#1 and other islands

\begin{tabular}{|cccccc|}
\hline island $\# 1$ & & & \multicolumn{2}{c|}{ Sum of island (2-11) } \\
Area & Height & Volume & Area & Height(average) & Volume \\
$\left(\mathrm{nm}^{2}\right)$ & $(\mathrm{nm})$ & $\left(\mathrm{nm}^{3}\right)$ & $\left(\mathrm{nm}^{2}\right)$ & $(\mathrm{nm})$ & $\left(\mathrm{nm}^{3}\right)$ \\
& $\mathrm{n} / \mathrm{a}$ & $\mathrm{n} / \mathrm{a}$ & $\mathrm{n} / \mathrm{a}$ & & $\mathrm{n} / \mathrm{a}$ \\
2510 & $2.23 / 2.59$ & 5890 & 8140 & 1.14 & 9260 \\
2687 & $2.95 / 3.46$ & 8354 & 10726 & 1.18 & 12613 \\
$\mathrm{~N} / \mathrm{A}$ & $3.24 / 3.74$ & $\mathrm{n} / \mathrm{a}$ & 8769 & 1.21 & 1.46 \\
3339 & $3.67 / 4.54$ & 13040 & 10823 & 1.68 & 20476 \\
3342 & $6.34 / 7.78$ & 22369 & 12217 & & 1.46 \\
\hline
\end{tabular}




\section{CHAPTER 6: HOW INDIUM GROWS ON THE INDIUM SUBMONOLAYER PHASES ON SI(111)}

\section{- DO WE NEED TO GROW ON THE ALPHA PHASE TO GET THE BEST QSE ISLANDS?}

(Based on a paper to published)

\section{Abstract}

Indium(In) is deposited on varies In phases(In beta phase, In $\sqrt{ } 31$ phase, In $4 \times 1$ phase and In 1x1R30 phase) at low temperature $(160 \mathrm{~K})$ and the low energy electron diffraction (LEED) and scanning tunneling microscope(STM) are used to study the growth of Indium. A novel phase transition from In beta and $\sqrt{3} 1$ phase to In 1 x1R30 phase is observed. The In atoms form fcc islands at low coverage on all the phases, but the fcc-bct transition is not observed in these substrate, which indicated that the In atoms on In substrates are very mobile and $\mathrm{Pb}$-alpha phase is very special substrate to grow In islands with QSE effects. Introduction

The In on $\mathrm{Si}(111)$ system is very rich in two-dimensional phases and has therefore attracted widespread attentions. Lander and Morrison's pioneering low energy electron diffraction (LEED) studies ${ }^{[1]}$ firstly discussed the In deposition on Si(111) surface and since then several phases are observed. Following this early work many laterally averaging studies are done but only a few resolving studies have been made of the In on $\mathrm{Si}(111)$ system. Although there is general agreement about several of the phases that appear with increasing coverage and temperature such as $7 \times 7, \sqrt{ } 3 \times \sqrt{ } 3-R 30^{\circ},(\sqrt{ } 31 \times \sqrt{ } 31), 4 \times 1$, several other phases are in question such as $2 \times 1,5 \times 5,(\sqrt{ } 43 \times 4),(4 \times 3 \sqrt{ } 3),(\sqrt{ } 7 \times \sqrt{ } 3)$, and $(1 \times 1)-R 30^{\circ}$. All the phases are named after their unit cell's size, for an example, $\sqrt{ } 3 x \sqrt{ } 3-R 30^{\circ}$ phase's unit cell is $\sqrt{ } 3$ times Si unit cell, and it is rotated $30^{\circ}$ from Si unit cells. In particular in the phases with coverage around one monolayer (ML), their coverage is not clearly understood yet. From the comprehensive scanning tunneling microscopy (STM) studies of Netzer's group ${ }^{[2-5]}$ the conclusion has been drawn that the (1x1)- $230^{\circ}$ structure does not exist but rather two different $(\sqrt{ } 7 x \sqrt{ } 3)$ structures exist that were not observed in diffraction experiments. More recently, the system has attracted attention again because it was suggested that one of its 
phases, the $4 \times 1$ structure, undergoes a charge density wave transition, a result that was later challenged. ${ }^{[6]}$ It is found that $\mathrm{Pb}$ islands grown on $\mathrm{In} 4 \mathrm{x} 1$ phase have extraordinary stability (Chpter.3).

Bauer's group ${ }^{[7]}$ made detailed researches about the structural phase transitions in the multiphase system indium on $\mathrm{Si}(111)$ as a function of coverage at different temperatures between -100 and $550{ }^{\circ} \mathrm{C}$ by low energy electron microscopy (LEEM) and low energy electron diffraction (LEED). Various In phases are made and these phases can be converted between each other according to the coverage at high temperature $\left(>300^{\circ} \mathrm{C}\right)$, However, there is no detailed study about the growth at low temperature yet. In this work we studied the growth of Indium on various phases of In at low temperature(170K).

Recently it is reported ${ }^{[8]}$ that when indium is deposited on In $4 \mathrm{x} 1$ phase at room temperature, the in will grow large islands but if In is deposited on the mixture of $\sqrt{3} 1 x \sqrt{3} 1$ phase and $4 \mathrm{x} 1$ phase, they will form nanowires, which indicates the boundary effects may also play a part.

\section{Experiments}

Experiments took place in a UHV chambers with base pressure $2 \times 10^{-11}$ Torr. The chamber is equipped with an Omicron SPA-LEED system, Auger spectrometer and mass spectrometer. The starting point of all experiments was the $\operatorname{Si}(111) 7 \times 7$ phase. This sample was prepared by flashing the crystal to $1250^{\circ} \mathrm{C}$, then cooling the crystal to $800^{\circ} \mathrm{C}$, the temperature of the $7 \times 7$ reconstruction. The different phases were obtained by depositing proper amount of In and annealing to $400^{\circ} \mathrm{C}$. The sample was then cooled to $170 \mathrm{~K}$ for further depositions. The deposition rate of In is approximately $0.1 \mathrm{ML} / \mathrm{min}$, which is calibrated by an oscillating quartz near the sample. The quartz's frequency is dependent on its own mass so when In is deposited on the sample, the In atoms will fall on to the surface and its mass will increase slightly (in the order of nanograms) and therefore change its frequency. By measure the change of its frequency we can measure the change of the quartz's mass therefore calibrate the flux rate of Indium. (see chapter. 2 for details about calibration) After each deposition 1D and 2D diffraction scans from the surface were taken to get the position and intensity of spots. Most of the scans are taken at electron energy E=38 
$\mathrm{eV}$ and for $1 \mathrm{x} 1 \mathrm{R} 30$ the scans are taken at $60 \mathrm{eV}$ because the spots have better intensity for the specific phase.

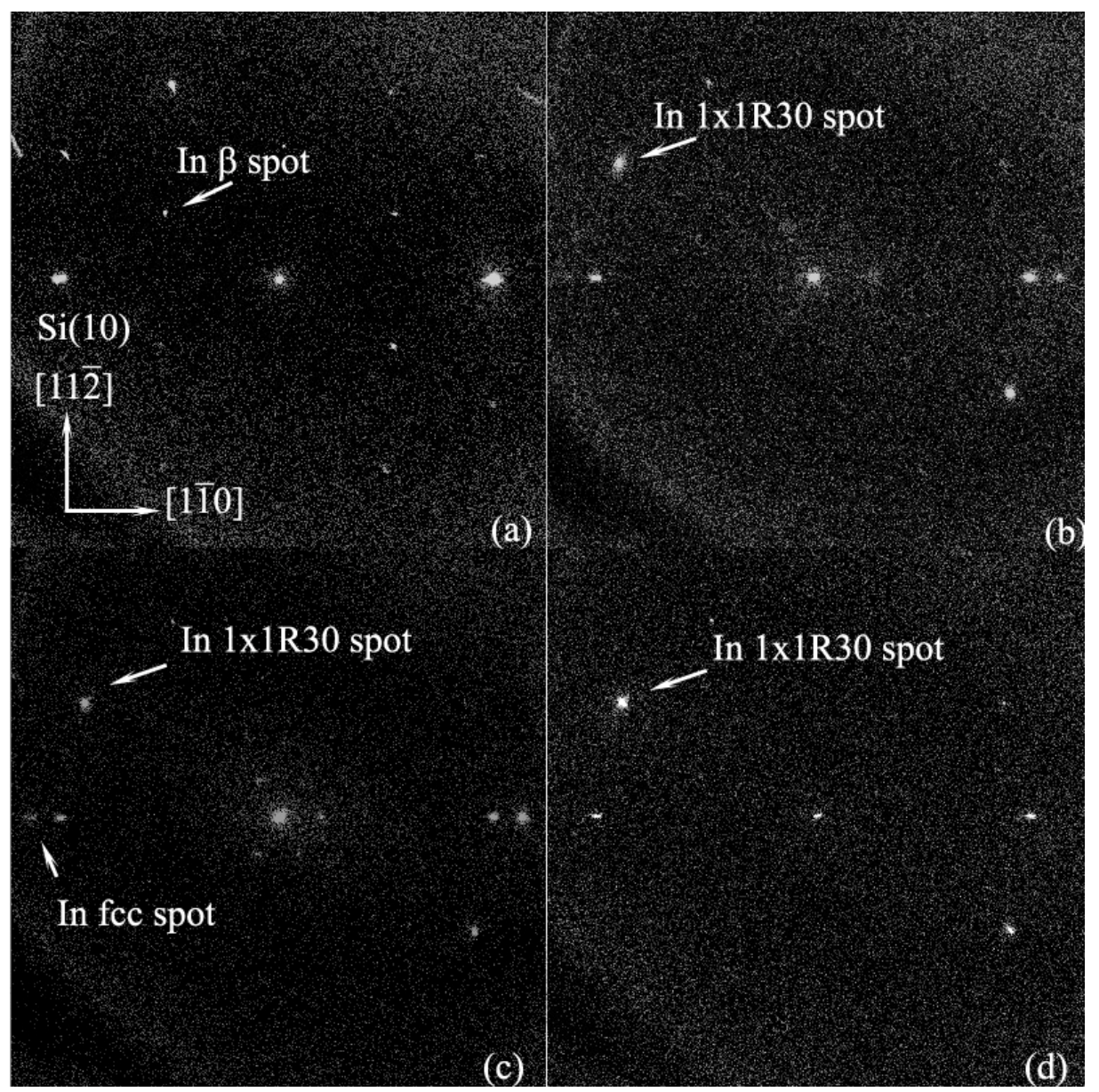

Figure $1240 \% \times 240 \% B Z 2 D$ diffraction patterns from SPA-LEED for In deposited on In $\beta$ phase. The major spots are marked in the figures. (a) In $\beta$ phase (b) after $1 \mathrm{ML}$ deposition (c) after $4 \mathrm{ML}$ deposition (d) after RT anneal

We also performed STM experiments that grow indium on indium $\beta$ phase, which is the indium phase with lowest coverage. The experiment took place in a separate UHV chamber with base pressure $2 \times 10^{-11}$ Torr equipped with an Omicron variable temperature STM. The starting point is $\operatorname{Si}(111) 7 \times 7$, too. The $\beta$ phases are made in similar way as the other chamber and cool down to $170 \mathrm{~K}$. And further Indium depositions are made. The flux rate of Indium is calibrated to be $0.1 \mathrm{ML} / \mathrm{min}$. The calibration of In flux is by integrating the total volume of In using the histogram after an amount of deposition (see Chapter.2 for more details). 
Results

1 Indium on indium $\sqrt{3} x \sqrt{ } 3(\beta)$ phase

We made the indium $\sqrt{3} x \sqrt{3}$ phase ( $\beta$ phase) by depositing $0.4 \mathrm{ML}$ of indium on $\mathrm{Si}$ $7 \times 7(111)$ and anneal to $400^{\circ} \mathrm{C}$. Then the system is cooled to $170 \mathrm{~K}$ for further depositions. Several depositions are made and the diffraction patterns are taken. The flux rate of indium is calibrated to $0.1 \mathrm{ML} / \mathrm{min}$.

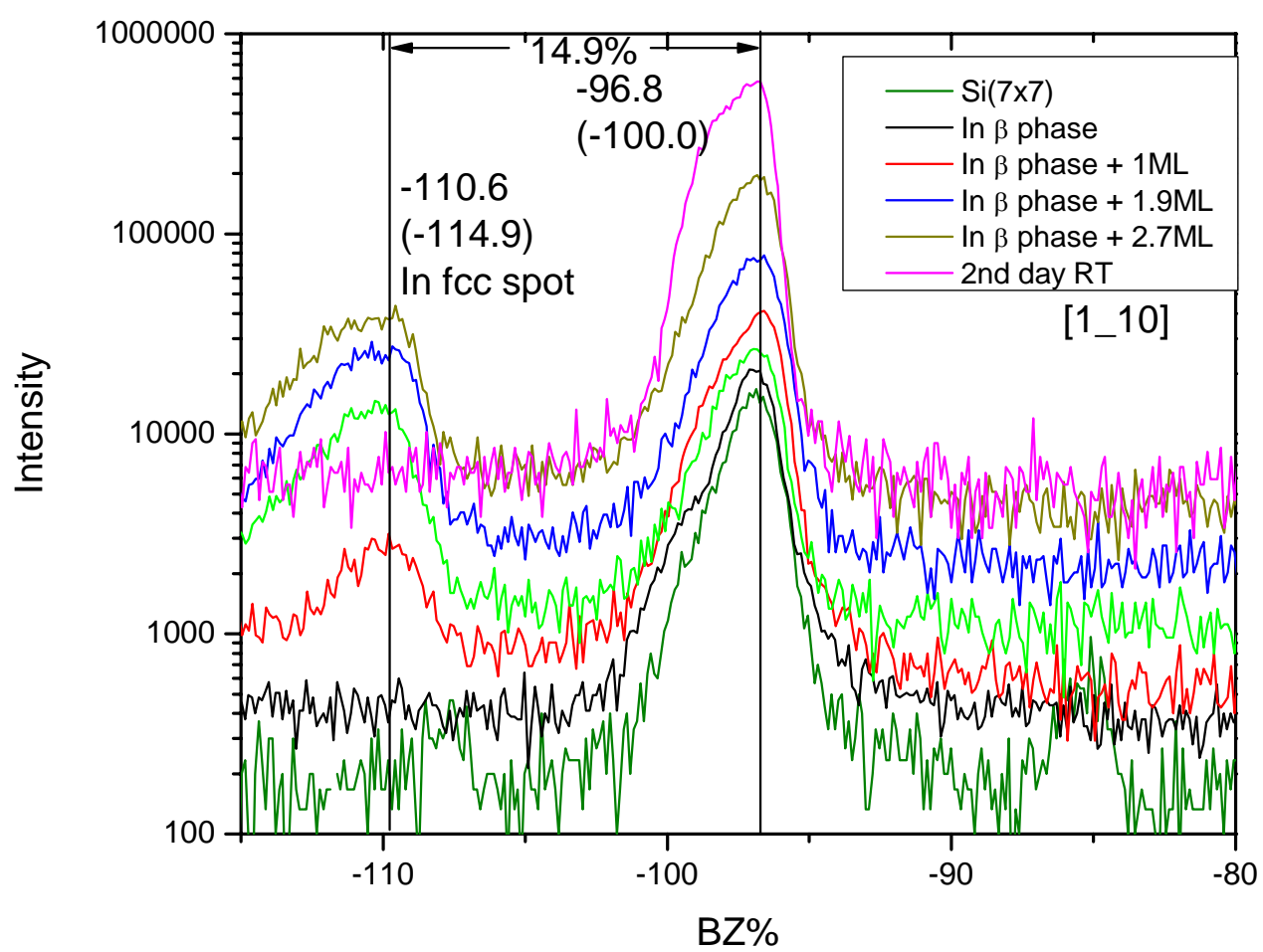

Figure $2151 \mathrm{D}$-scans near $100 \% \mathrm{BZ}$ range for In on In $\beta$ phase in [1 $\overline{1} 0]$ direction. Scans at different coverage are drawn in different colors and the curves are displaced in vertical direction to avoid overlapping. The $\mathrm{Si}(7 \times 7)$ curve is included for calibration. The positions of (10) spot(-100) and fec spot(114.9) are marked in the figure. The numbers in parentheses are corrected. The figure shows that the fcc spots intensity grows with coverage. Temperature is $170 \mathrm{~K}$.

Fig. 1 shows the $240 \% x 240 \%$ BZ 2D scan images at different coverages. Fig.1(a) is the original indium $\beta$ phase. The $\sqrt{3} \mathrm{x} \sqrt{3}$ lattice is $\sqrt{3}$ times silicon lattice in real space so its unit cell is $1 / \sqrt{ } 3$ of Silicon's. ( $\mathrm{Si}(10)$ spot is also marked in Fig.1(a)) in reciprocal space. And it is $30^{\circ}$ rotated from Si lattice. Due to the 6-fold symmetry, the direction of $\sqrt{3} x \sqrt{3}$ spot marked in Fig.1(a) is equivalent to the $[11 \overline{2}]$ direction. Fig. 1(b) is taken after 1ML of In 
deposition on $\beta$ phase. The $\beta$ spots disappear completely and a spots in the $[11 \overline{2}]$ direction appear. This spot is at 103\%BZ from the (00) spot in 1-D scan (see details in Fig 2) and belongs to the $1 \times 1$ R30 phase. This means that $\beta$ phase is converted to 1x1R30 phase. Fig. 1(c) is taken after $4 \mathrm{ML}$ of indium deposition totally on $\beta$ phase. We can see there is a strong spot in $[1 \overline{1} 0]$ direction at $115 \%$ outside $\operatorname{Si}(10)$ spot. It is the spot from In islands in face-centered cubic (fcc) structure. The 1x1R30 spots are still strong. Between 1ML and 4ML there is no qualitative change in the diffraction, the fcc spot becomes stronger with coverage increases. Fig.1(d) is taken after overnight room temperature annealing. The 1x1 spots is maintained but the fcc spots disappear. From above, we can tell that the 1x1 R30 phase is stable even at room temperature. The fcc structure is not stable at RT. It is well known that the bulk structure of In is body-centered tetragonal (bct).

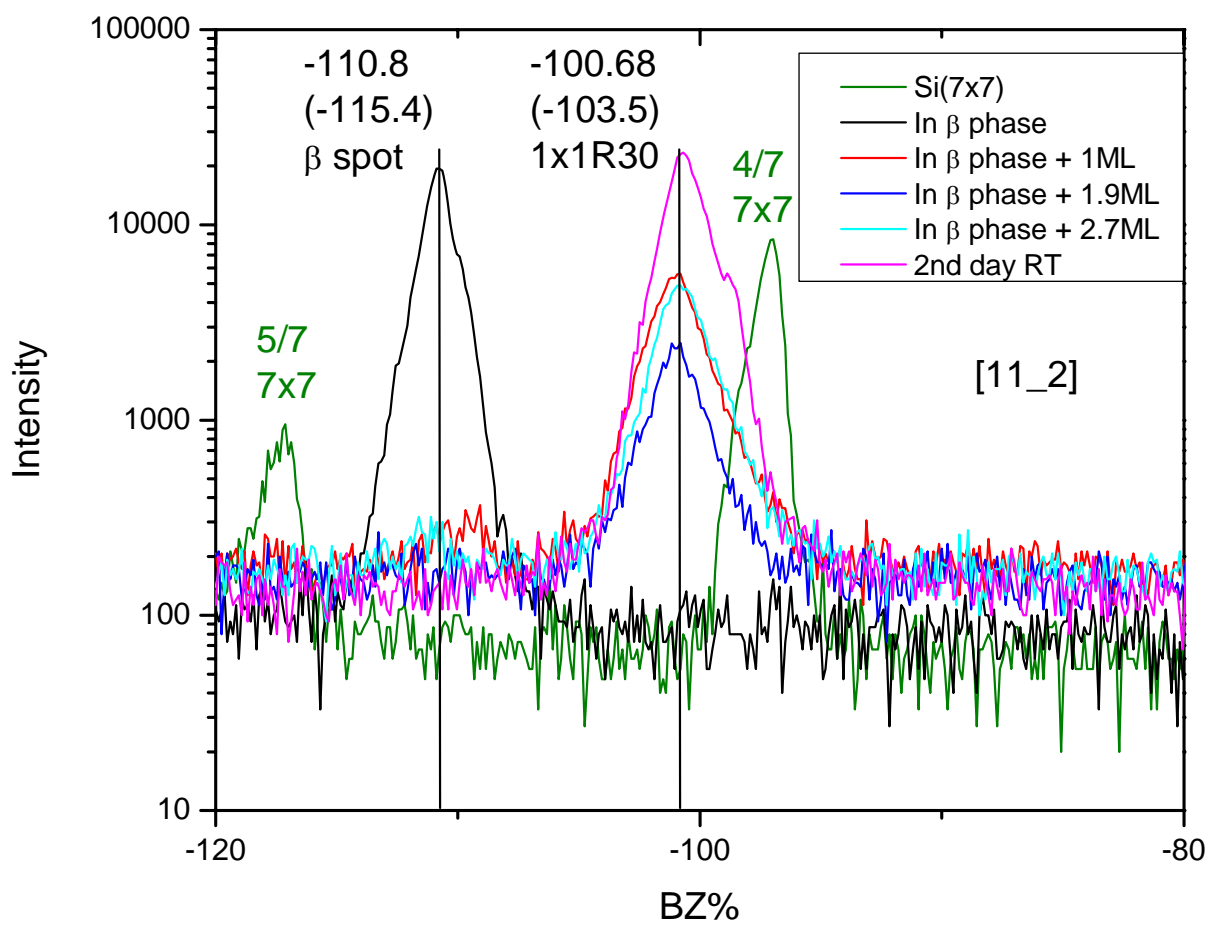

Figure 3 1D-scans near $100 \% \mathrm{BZ}$ range for In on In $\beta$ phase in [11 $\overline{2}]$ direction. Scans at different coverage are drawn in different colors. The $\operatorname{Si}(7 \times 7)$ curve is included for calibration. The positions of 1x1R30 spot(-103.5) and $\beta$ spot(-115.4) are marked in the figure as well the $7 \times 7$ spots. The numbers in parentheses are corrected. The figure shows that the beta spots disappear after the first deposition and 
1x1R30spots appear. The intensity of $1 \times 1$ spot is not directly dependent on the coverage and it becomes even larger at RT. Temperature is $170 \mathrm{~K}$.

Fig. 2 and Fig. 3 give the 1-D scans in $[1 \overline{1} 0]$ and $[11 \overline{2}]$ directions. Compared to 2D scans, the 1D scans have longer collection time window therefore the fluctuation is less and the random error is much lower compared to $2 \mathrm{D}$ scans, so we can get data with better accuracy to find the position of spots. It might be good to mention that all the curves in the images are from the raw data, which need to be corrected to remove the system error. This is how the correction works: The exact positions of the $7 \times 7$ spots are well known, i.e. $1 / 7,2 / 7, \ldots$ of $100 \% \mathrm{BZ}$, or $14.29 \%, 28.57 \% \ldots$ but the readings from the scans are not exactly these numbers due to system errors. For the same reason, the In fcc position from the raw data is not its really position and need to be corrected. We use the simplest way to correct this error by linear interpolation. For an example, The reading for $\operatorname{Si}(7 \times 7)$ spots reading are:-96.8 (7/7) and -109.3 (8/7) but their actual positions are -100(7/7) and 114.3(8/7). For the fcc spot (-110.6) near (8/7) spot, its real position is -[(110.6109.3)/(109.3-96.8)+8]/7*100\%=-115.7\%. All the other corrections are done in similar way. All the positions are marked by the both raw and corrected value(in parentheses) in 1D scans.

In Fig. 2 are the 1D scans in $[1 \overline{1} 0]$ direction near $100 \%$ BZ taken from Indium deposition experiments on In $\beta$ phase. The depositions are done at $\mathrm{T}=170 \mathrm{~K}$. Each curve is taken from a certain coverage, which is shown in the legend. There are two peaks in this region. One is at $-100 \% \mathrm{BZ}$, which is the (10) Si spot the other one is at $-115.7 \%$. Fig.2 shows that this spot appears after $1 \mathrm{ML}$ of deposition and becomes stronger with coverage increasing until 4ML. After room temperature annealing, it disappears. From its position ${ }^{[14]}$ and behavior we can tell it is the In fcc (10) spots. In Fig.3 are the 1D scan in [11 $\overline{2}]$ direction near $100 \% \mathrm{BZ}$. There are two peaks can be seen in this region. One is at $-115.4 \%$, which is from the beta phase $(2 / 3$ spot at $2 \sqrt{ } 3 / 3 * 100 \% \mathrm{BZ})$. It disappears after the first deposition. The other one is at $-103.5 \% \mathrm{BZ}$. It appears after the first deposition and with coverage increasing it become strong and stay even at room temperature. It is from the $1 \mathrm{x} 1 \mathrm{R} 30$ phase, a 2-D In phase which has higher coverage than beta phase. ${ }^{[6]}$ The $1 \mathrm{x} 1$ phase's unit cell is very close to silicon unit cell(103\%BZ), and it coverage is $1.05 \mathrm{ML}$. The clean $7 \mathrm{x} 7$ curve is also included for correction purpose. From this experiment we can tell that with In deposition, the In beta 
phase convert to $1 \times 1$ R30 phase. After the 1x1R30 phase forms, In islands with fcc structure grow on it. The 1x1R30 phase is stable at Room Temperature, while In fcc islands are not.

2 In on In $\sqrt{ } 31 x \sqrt{ } 31$ phase

We made In $\sqrt{ } 31 \mathrm{x} \sqrt{3}$ phase by depositing $\sim 0.8 \mathrm{ML}$ of indium on $\operatorname{Si}(111)$ reconstruction and annealing to $300^{\circ} \mathrm{C}$. Then we make several depositions at low temperature(170K). The In flux rate is $\sim 0.1 \mathrm{ML} / \mathrm{min}$. Fig. 4 shows an $\operatorname{In} \sqrt{ } 31 \times \sqrt{ } 31$ phase model in real space, there are 17 In atoms in each $\sqrt{ } 31 x \sqrt{ } 31$ unit cell ${ }^{[9]}$. Therefore the coverage of In $\sqrt{ } 31 \mathrm{x} \sqrt{ } 31$ phase is $17 / 31=0.53 \mathrm{ML}$

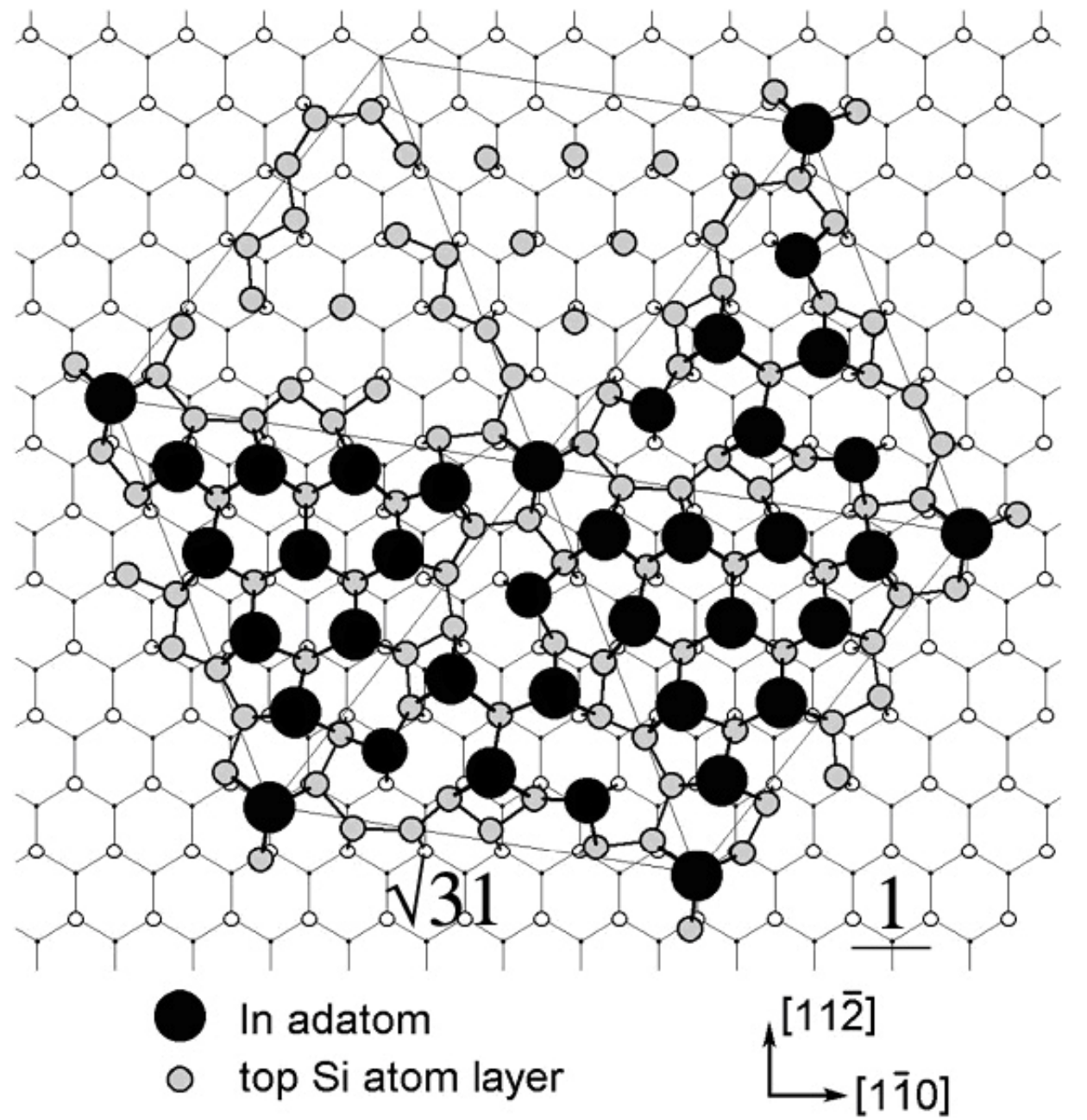

Figure 4 structure of In $\sqrt{ } \mathbf{3 1}$ phase. taken from [9] The dark back circles are the In atom, hexagonal the unit cell is shown in the figure. The length of the hexagonal is $\sqrt{ } \mathbf{3 1}$ times the silicon cell.

Fig. 5 shows the $240 \%$ x $240 \%$ BZ 2D scans of different stages of the deposition.

Fig.5(a) shows the $\sqrt{ } 31$ phase before depositions. Its wave vector is $18 \%(1 / \sqrt{ } 31 * 100 \%) \mathrm{BZ}$ 
in reciprocal space and is $9^{\circ}$ off the $[1 \overline{1} 0]$ direction. Fig.5(b) is taken after $0.5 \mathrm{ML}$ of In deposition. The $\sqrt{ } 31$ spots have nearly vanished and the $1 \times 1 R 30$ spot in $[11 \overline{2}]$ direction shows up. This means that most of $\sqrt{ } 31$ phase has converted into $1 \times 1 \mathrm{R} 30$, and there is a strong 1x1R30 spot in [11 $\overline{2}$ ] direction. Fig.5(c) is taken after 4ML of In deposition. The fcc spots show up which means there are already In islands. Fig.5(d) is taken after room temperature annealing. $\sqrt{ } 31$ phase and fcc spots disappear, and 1x1R30 spots is still strong.

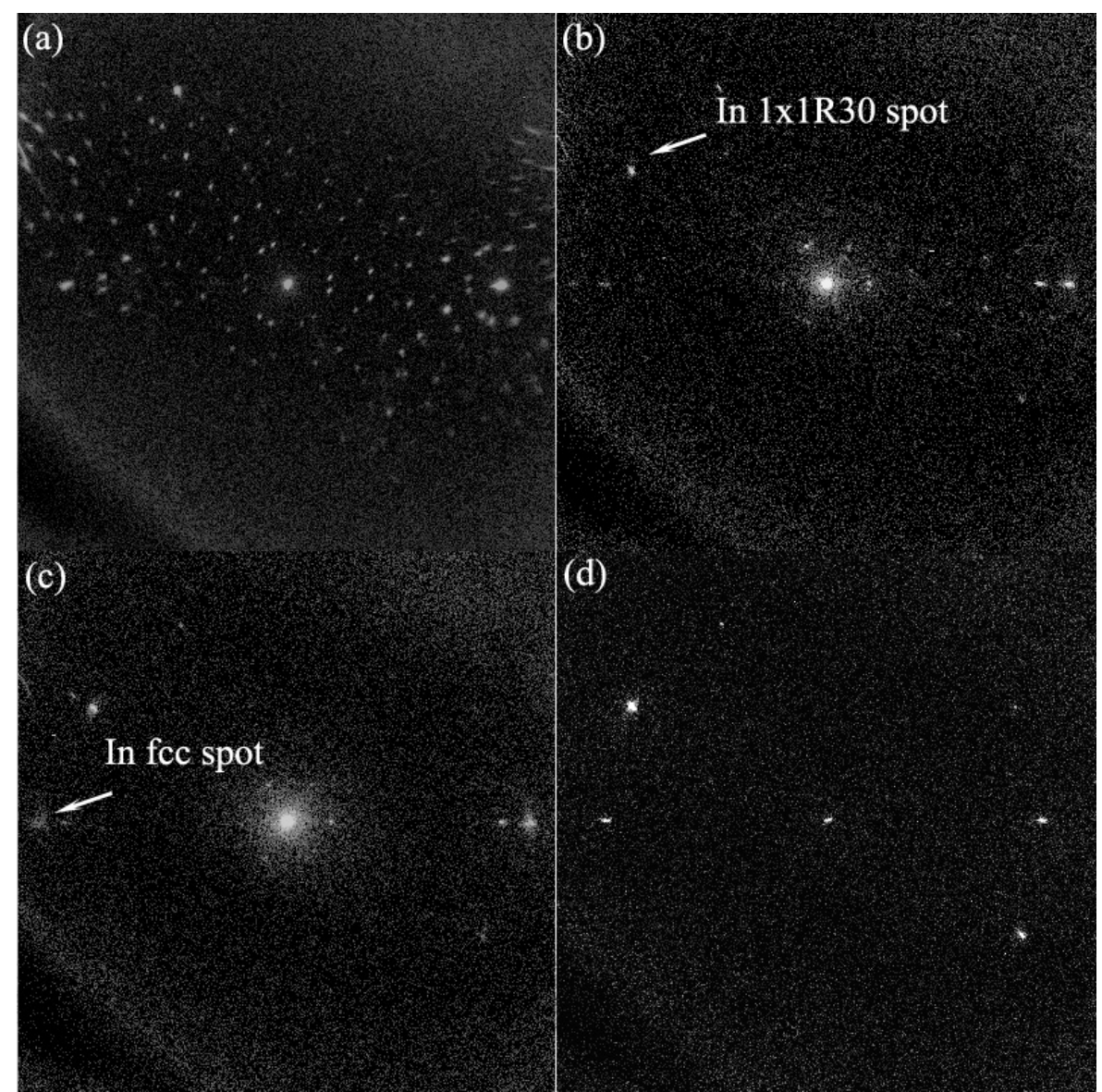

Figure 5 240\%x240\%BZ 2D diffraction patterns from SPA-LEED for In deposited on In $\sqrt{ } 31 x \sqrt{ } 31$ phase. The major spots are marked in the figures. (a) In $\sqrt{31} \times \sqrt{31}$ phase, $T=170 \mathrm{~K}$ (b) In $\sqrt{31}$ phase $+0.5 \mathrm{ML}$ of In, $\mathbf{T}=170 \mathrm{~K}$ (c) In $\sqrt{31}$ phase $+4 \mathrm{ML}$ of In, $T=170 \mathrm{~K}$ (d) After RT annealing

Fig.6 shows the 1D scans near $100 \% \mathrm{BZ}$ in [1 $\overline{1} 0]$ direction. The $1-\mathrm{D}$ profile at different coverages are displayed in different colors and the position of the major peaks are marked, the values in parentheses are the corrected.. The curves are similar to the In on In beta phase experiments. There are two peaks, $\operatorname{Si}(10)$ and In fcc(10) spot. The fcc peak appears in $[1 \overline{1} 0]$ direction after $1 \mathrm{ML}(10$ minutes) of deposition and disappear after room 
temperature annealing. The fcc spots is at $-115.7 \%$, which is the same spot we see in the In on $\mathrm{Pb}$ alpha phase and In on In $\beta$ phase experiments.

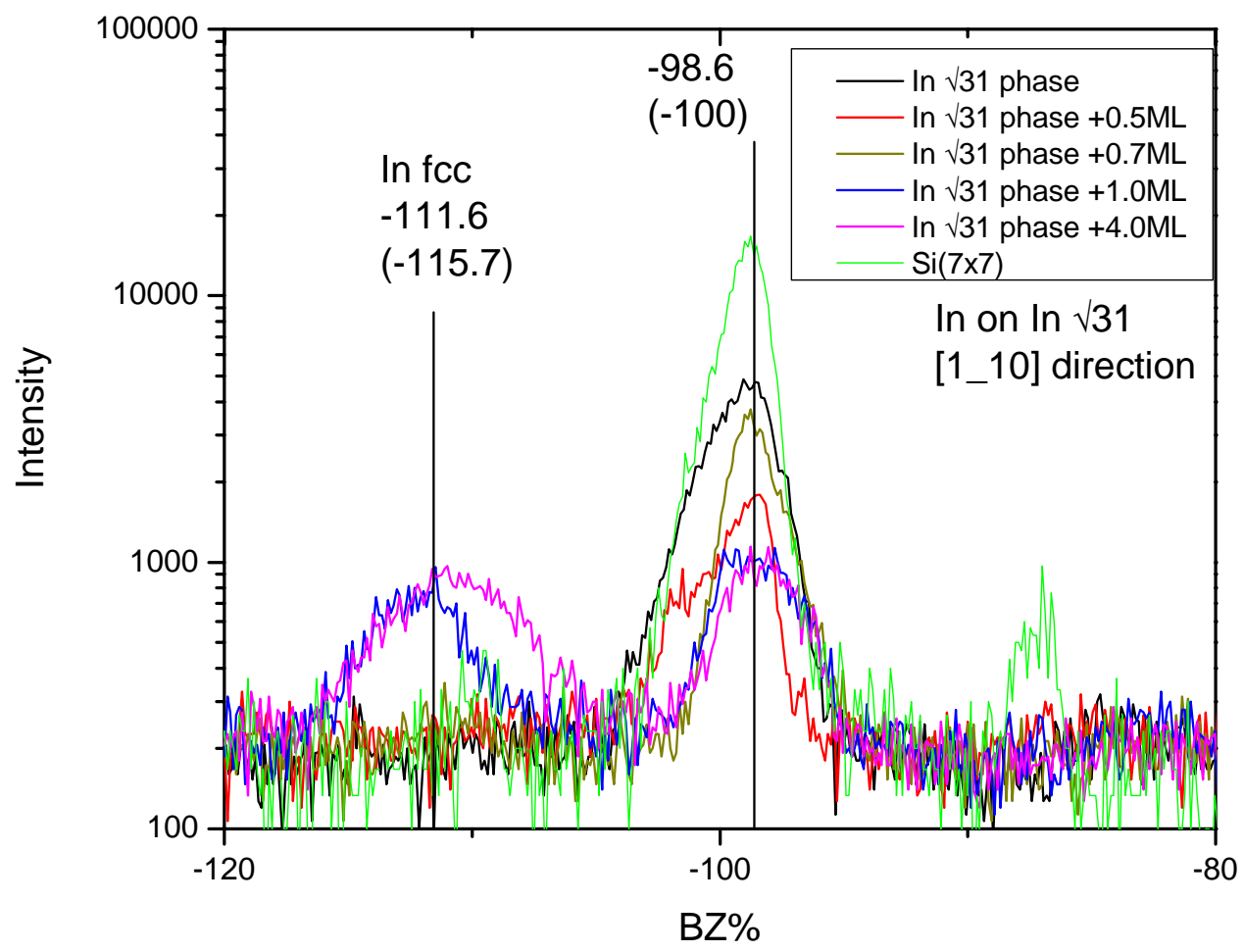

Figure 6 1D-scans near $100 \% B Z$ range for In on In $\sqrt{3} 1$ phase in [1 $1 \overline{1} 0]$ direction. Scans at different coverage are drawn in different colors. The $\mathrm{Si}(7 \times 7)$ curve(green) is included for calibration. The positions of (10) spot(-100) and fec spot(-115.7) are marked in the figure. The numbers in parentheses are corrected values. The figure shows that there are also In fec spots when we put In on $\sqrt{31}$ phase. Temperature is $170 \mathrm{~K}$.

Fig. 7 shows the $1 \mathrm{D}$ scans in $[11 \overline{2}]$ direction. The $-116.5 \%$ spot belongs to $\sqrt{ } 31$ phase, it disappears after the first In deposition, therefore the $\sqrt{ } 31$ phase doesn't exist already. At the same time, the $1 \times 1 \mathrm{R} 30$ spot at $-103 \% \mathrm{BZ}$ appears. At this time, there is no islands yet (there is no fcc spot in $[1 \overline{1} 0]$ direction) which means the $\sqrt{ } 31$ phase is converted into $1 \times 1$ R30 phase. The 1x1R30 spots didn't disappear after further deposition (4ML) or room temperature annealing, which is similar to the experiments on In $\beta$ phase. From this experiment we can tell, like the In $\beta$ phase, the $\sqrt{ } 31$ phase will convert to $1 \times 1 \mathrm{R} 30$ phase before islands grow, too. 


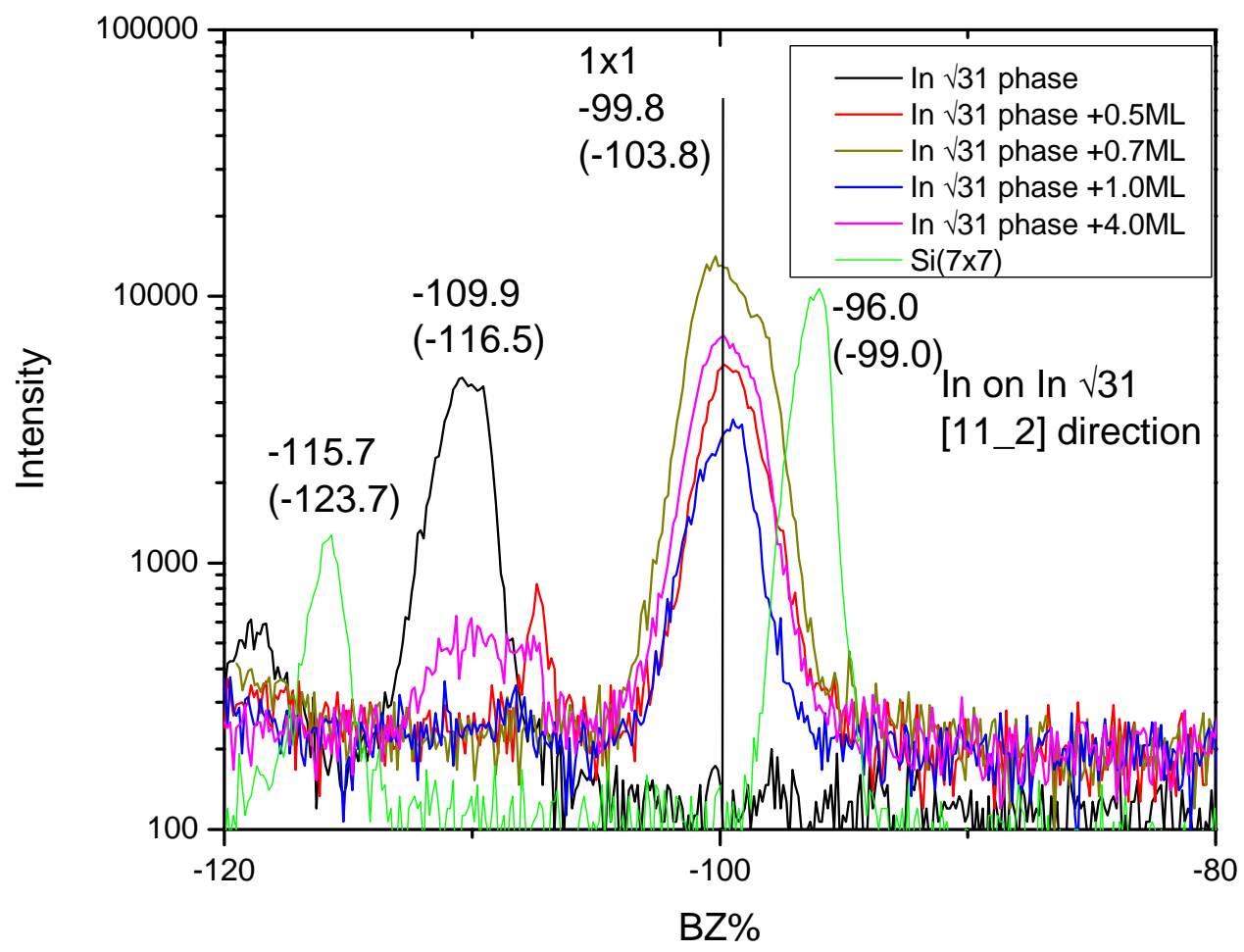

Figure 7 1D-scans near $100 \% \mathrm{BZ}$ range for In on In $\sqrt{31}$ phase in $[11 \overline{2}]$ direction. Scans at different coverage are drawn in different colors. The $\mathrm{Si}(7 \times 7)$ curve(green) is included for calibration. The positions of the $1 \times 1$ spots(-103.8) are marked in the figure. The numbers in parentheses are corrected values. Temperature is $170 \mathrm{~K}$.

3 In on In $4 x 1$ phase

We also tried to make several depositions at low temperature $(170 \mathrm{~K})$ on In $4 \mathrm{x} 1$ phase. The In $4 \times 1$ phase has coverage of $1 \mathrm{ML}$. The In flux rate is $\sim 0.1 \mathrm{ML} / \mathrm{min}$.

Fig 8 shows the $240 \%$ BZ 2D diffraction patterns after various depositions. Fig.8(a) is the starting In $4 \times 1$ phase. In reciprocal space the $4 \times 1$ structure's unit cell is $25 \% \mathrm{x} 100 \%$. In [1 $\overline{1} 0]$ direction the spacing between neighbor $4 \times 1$ spots is $25 \%$. The angle between the two unit vectors is $60^{\circ}$. F-ig.8 (b) is taken after a small amount of deposition (1ML), The $4 \mathrm{x} 1$ spots become weaker because the surface is covered by the wetting layer. Fig.8(c) is taken

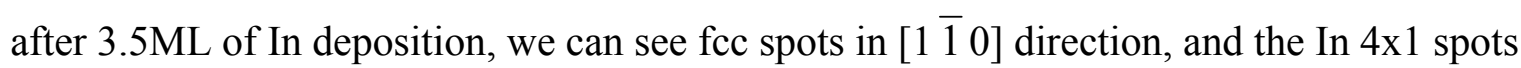
almost disappear. There are also some spots near (00) spot, they are corrugation from the top 
of In islands. We know that the lattice constant of In is different from the $\mathrm{Si}$, so there is mismatch between the two lattice. The corrugation pattern is actually the Moire pattern due to the mismatch. In fig.1(c) and fig.5(c) we see the corrugation patterns, too, but they are not as strong as on In $4 \times 1$. Fig.8(d) is taken after room temperature annealing. The fcc and corrugation spots are gone and 4x1 spots are back. Therefore, the In wetting layer have moved away and the $4 \times 1$ phase is revealed again. In this experiment the $1 \times 1 \mathrm{R} 30$ spots is not observed, so the $4 \times 1$ phase doesn't convert into 1x1R30.

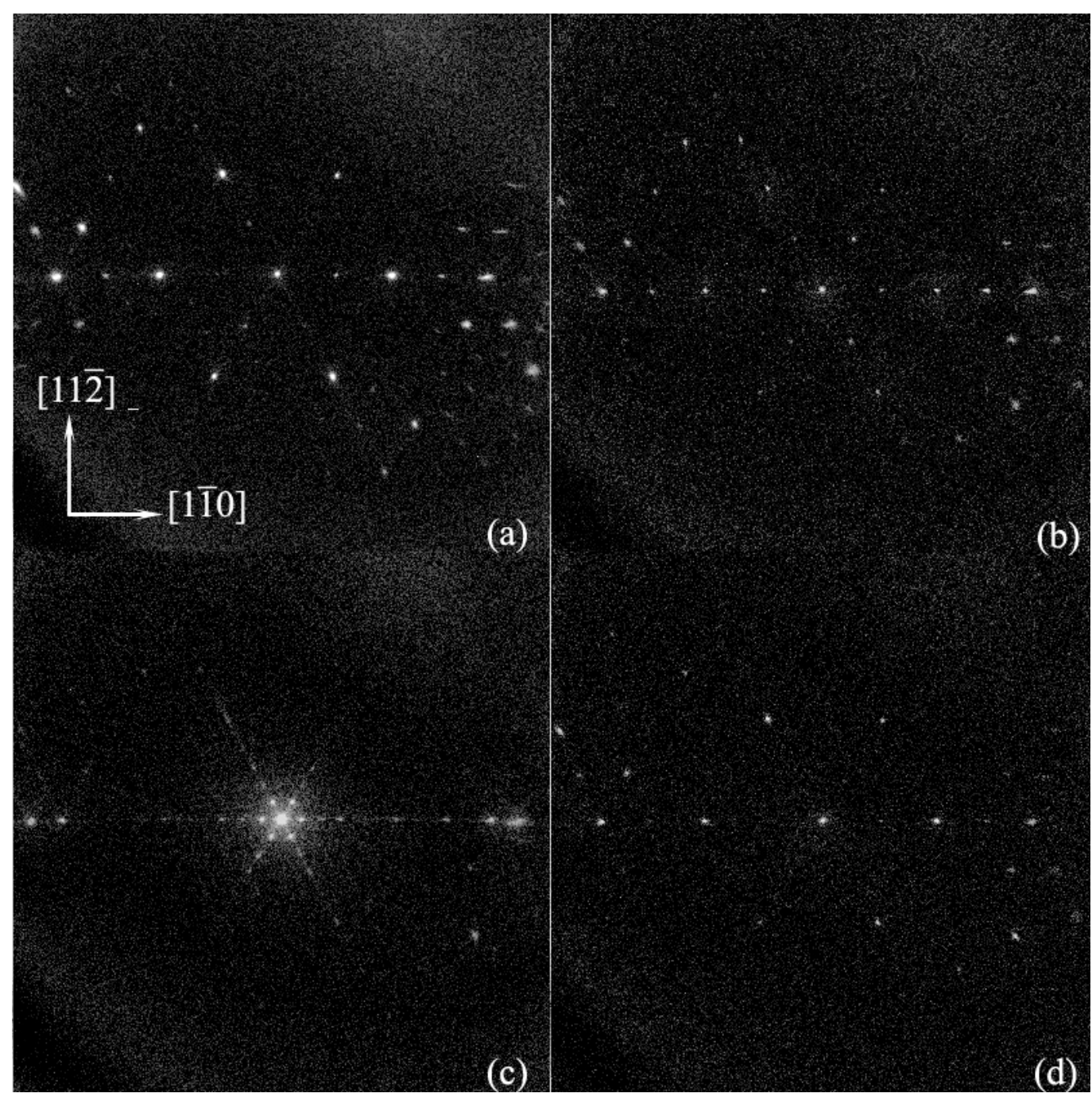

Figure $8240 \% \times 240 \% B Z 2 D$ scans for In deposited on In 4x1 phase. (a) In 4x1 T=170K (b)In 4x1 +1ML deposition $\mathrm{T}=170 \mathrm{~K}$ (c) In $4 \times 1+3.5 \mathrm{ML}$ deposition $\mathrm{T}=170 \mathrm{~K}$ (d) after room Temperature annealing Fig.9 shows the 1D scan along [1 $\overline{1} 0]$ direction near $-100 \% \mathrm{BZ}$. The profiles at different coverages are displayed in different colors. The $4 \mathrm{x} 1$ spots at $-75 \%$ can be seen at all coverages. In fcc spot(-115\%) shows up after $1 \mathrm{ML}$ deposition, and disappears after room 
temperature annealing, like previous experiments. Fig.10 shows the 1D scan along [11 $\overline{2}]$ direction near $100 \% \mathrm{BZ}$. We find a $1 \times 1 \mathrm{R} 30$ spots at $-103 \%$, which is too weak to be visible in the 2D pattern. Due to its weakness, we don't think it is converted from $4 \times 1$ phase. We can also see a spot at $-86.6 \%(\sqrt{ } 3 / 2)$ which is the (11) spot from $4 \times 1$ phase. This experiment shows that the $4 \mathrm{x} 1$ spot is stable. When we make depositions, the In islands grows on In $4 \mathrm{x} 1$ phase but the $4 \times 1$ phase is not destroyed. In $4 \times 1$ phase is revealed again after the In islands coarsening at room temperature.

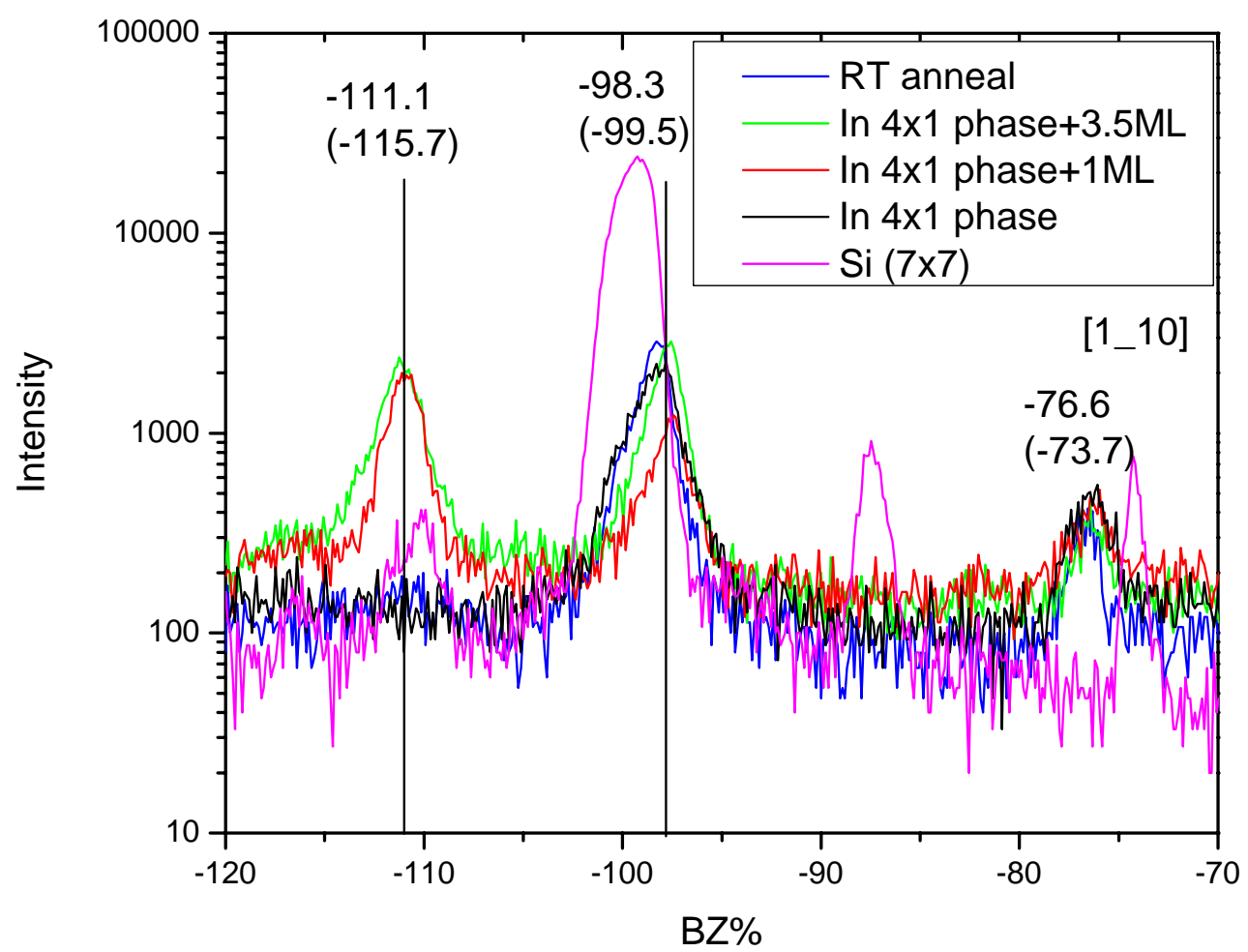

Figure 9 1D-scans near $100 \% \mathrm{BZ}$ range for In on In 4x1 phase in [1 $\overline{1} 0]$ direction. Scans at different coverage are drawn in different colors. The $\mathrm{Si}(7 \times 7)$ curve(magenta) is included for calibration. The positions of the $4 \times 1$ spots(-73.7,-99.5) and the fec spot $(-115.7)$ are marked in the figure. The numbers in parentheses are corrected values. Temperature is $170 \mathrm{~K}$.

4 In on In $1 \times 1 R 30$ 
We also try to make several depositions on In 1x1R30 phase at low temperature (170K). The In 1x1 R30 phases is made by depositing $1.2 \mathrm{ML}$ of indium on Si $7 \mathrm{x} 7$ phase and then annealing to $300^{\circ} \mathrm{C}$. The In flux rate is $\sim 0.1 \mathrm{ML} / \mathrm{min}$.

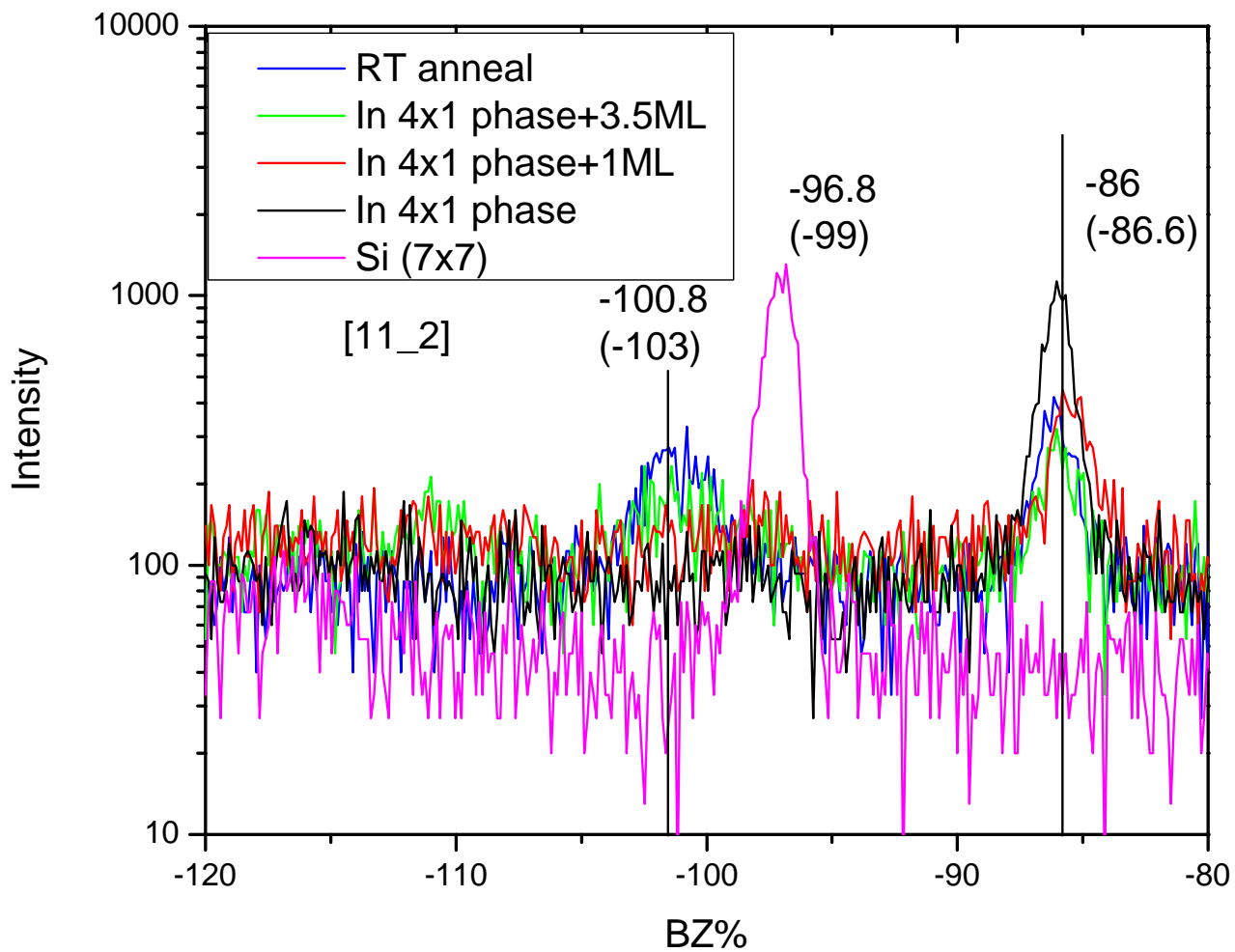

Figure 10 1D-scans near 100\%BZ range for In on In 4x1 phase in [11 $\overline{2}]$ direction. Scans at different coverage are drawn in different colors. The $\mathrm{Si}(7 \times 7)$ curve(magenta) is included for calibration. The positions of the $1 \times 1(-103)$ and $4 \times 1$ spots $(-86.6)$ are marked in the figure. The numbers in parentheses are corrected values. Temperature is $170 \mathrm{~K}$.

Fig. 11 shows the $240 \%$ 2D scan images at different stages. Fig 11(a) is the original $1 \times 1 R 30$ phase. We can see strong $103 \%$ spot in[11 $\overline{2}]$ direction. Fig.11(b) is taken after 1.2ML of In deposition, the $1 \mathrm{x} 1$ spots remains and fcc spots appear. We can also observe there is a $10 \times 10$ superstructure (wave vector $10 \%$ ) near the (00) spot, which is the corrugation. Fig.11(c) is taken after 4ML of In deposition. The fcc spots become stronger and the corrugation patterns is still visible. Fig.11(d) is taken after overnight room temperature annealing. The fcc and corrugation spots disappear, and the $1 \mathrm{x} 1$ spots still remains. The 
superstructure disappears with annealing. They show up and disappear with fec spots, this is the why we conclude they are from the In fcc islands.

Fig. 12 is the $1 \mathrm{D}$ scan in [1 $\overline{1} 0]$ direction near $100 \%$. Profiles at different coverages are plotted in different colors. We can see the fcc spots at $-115 \%$, which is consistent with the fcc indium islands position in the indium on $\mathrm{Pb}$ alpha phase experiments. The fcc spots shows up after only after $0.4 \mathrm{ML}$, which means 1x1R30 is almost "saturated" and the incoming In atoms goes to islands directly. Fig. 13 is the 1D scan in [11 $\overline{2}]$ direction. The $1 \times 1$ spot is at $-103 \%$. The clean $7 \times 7$ curve is also included for correction purpose. We find the fcc spot is also dependent on the coverage and disappear after RT annealing, while the 1x1R30 spots is not. The $1 \mathrm{D}$ scan results are consistent with $2 \mathrm{D}$ scans. 


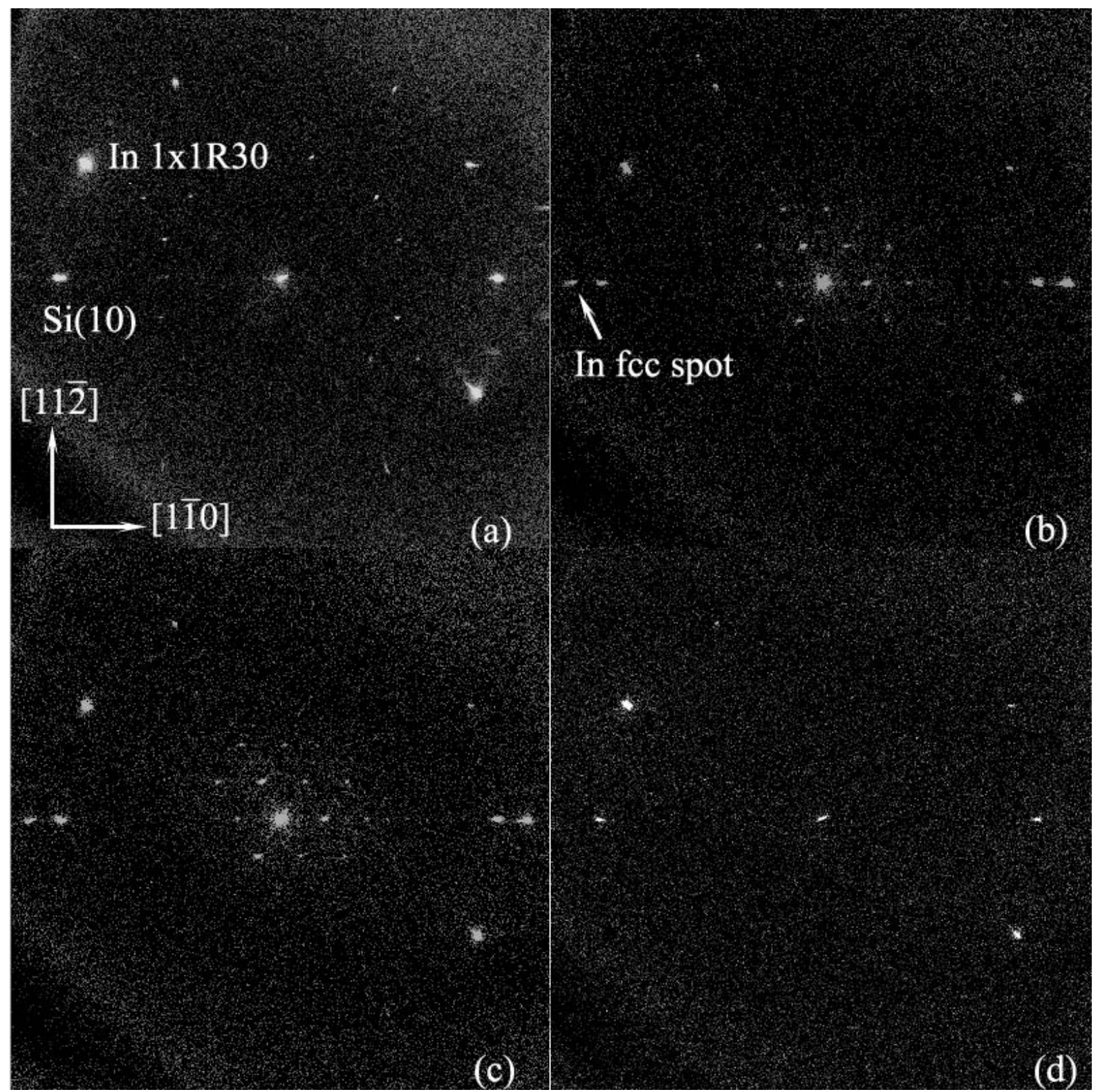

Figure $11240 \% x 240 \% B Z 2 D$ scans for In deposited on In 1x1 R30 phase. (a) In 1x1R30, T=170K (b) In $1 \times 1 R 30+1.2 M L$ of In, $T=170 K$ (c) $\operatorname{In} 1 \times 1 R 30+4 M L$ of $I n, T=170 K$ (d) after $R T$ anneal 


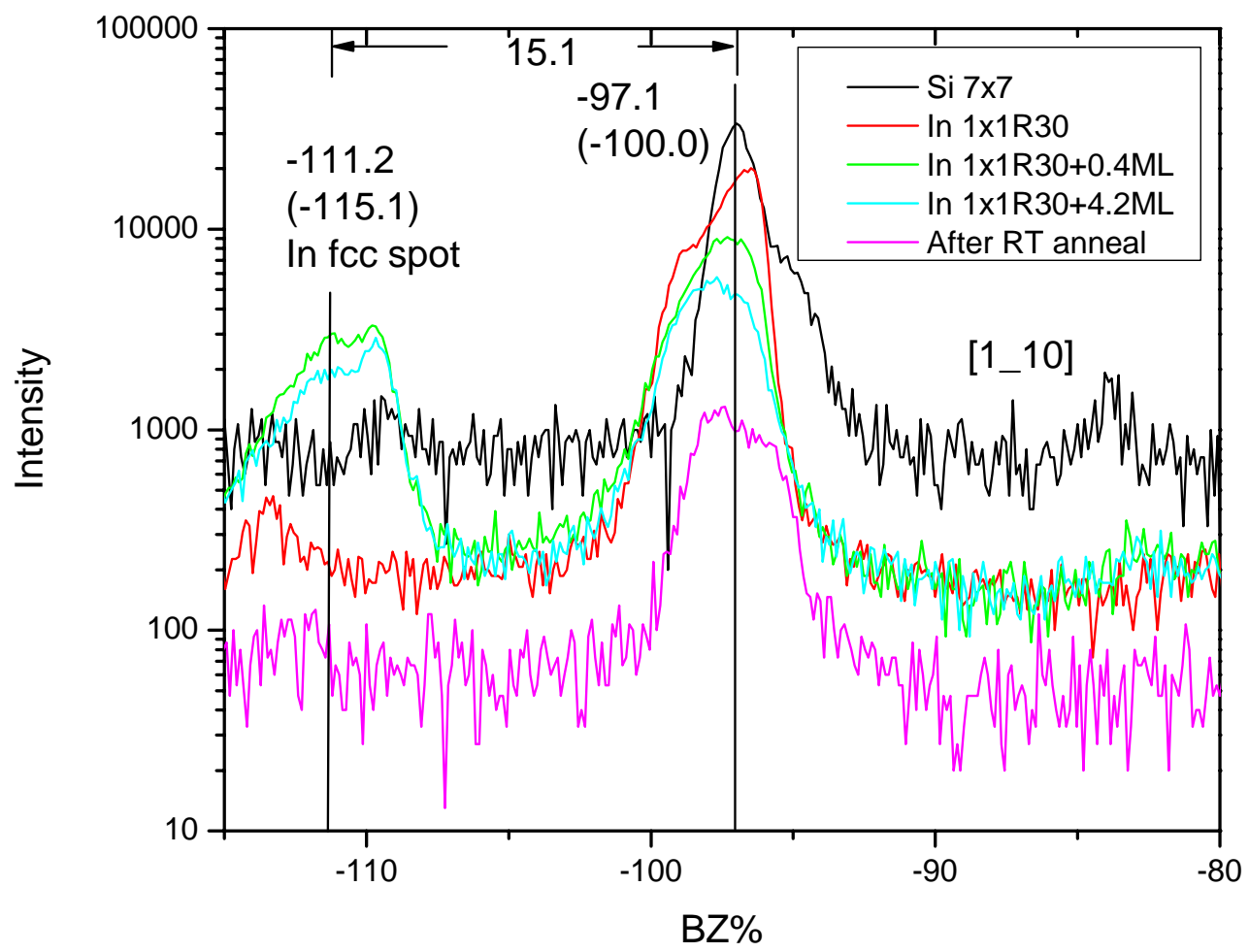

Figure 12 1D-scans near 100\%BZ range for In on In 1x1R30 phase in [1 $1 \overline{1} 0]$ direction. Scans at different coverage are drawn in different colors. The $\mathrm{Si}(7 \times 7)$ curve(black) is included for calibration. The positions of the (10) (-100) and In fec spots (-115.1)are marked in the figure. The numbers in parentheses are corrected values. Temperature is $170 \mathrm{~K}$. 


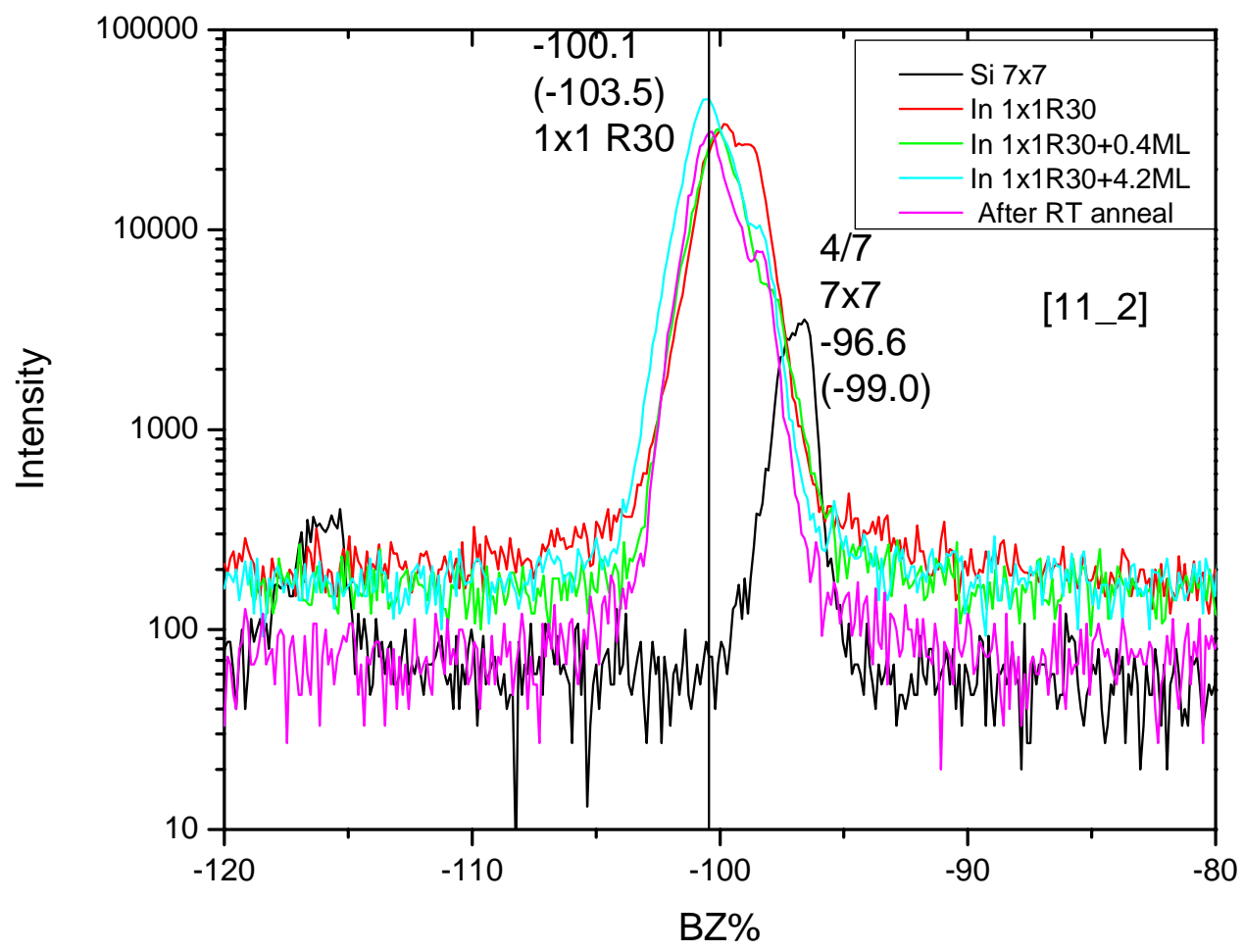

Figure 13 1D-scans near $100 \% \mathrm{BZ}$ range for In on In 1x1R30 phase in [11 $\overline{2}]$ direction. Scans at different coverage are drawn in different colors. The $\mathrm{Si}(7 \times 7)$ curve(black) is included for calibration. The positions of the $1 \times 1 R 30 \operatorname{spot}(-103.5)$ and the $S i 7 x 7 \operatorname{spot}(-99.0)$ are marked in the figure. The numbers in parentheses are corrected values. Temperature is $170 \mathrm{~K}$.

\section{STM experiments}

We also make the depositions experiments in STM experiments. Fig.14(a) shows 2.7ML of In deposited on In $\beta$ phase at 180K. On the surface exist two different types of islands. One is the huge rectangle-shaped island, the other is the much lower and smaller islands. In Fig 14(b) is the 1-D profile along the arrow, we can the height of the two types of islands have huge difference. The small islands are 1.2-1.9nm hight, corresponding 4-7 layer-high fcc islands. The huge island in rectangle shape is bct island. The STM experiments show that bet islands exist when we deposit In on In substrate. The main difficulty in STM experiments are the bct islands grow too fast and it is very hard to get a good images of decent quality without polluting the tip. 

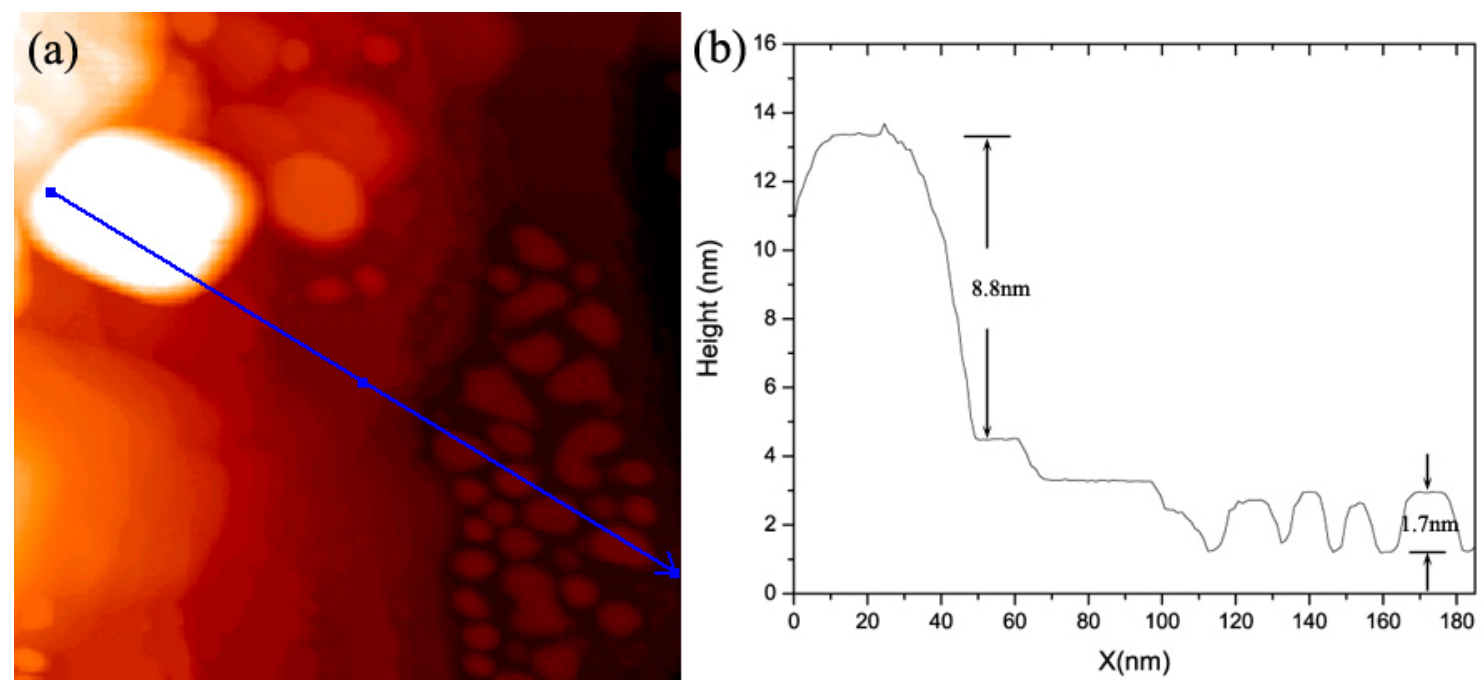

Figure 14 (a) STM image with 2.7ML of In on In beta phase. Image size 170nmx170nm T=180K (b) 1D profile along the arrow in (a).

Discussion

1 1x1R30 phase

There is some controversy about the existence of the 1x1R30 phase. The STM studies by Netzer's group think it is a pseudomorphic In overlayer which is only stable near defects. ${ }^{[10]}$ They showed that the diffraction spots attributed to this structure are due to the presence of several domains of a $\sqrt{7} x \sqrt{3}$ structure. They found two different domains: a nearly hexagonal one with coverage $1 \mathrm{ML}$ and a nearly rectangular one with coverage 1.2 ML. Bauer's group claims it is a slight distorted 1x1(by 3.85\%) and not exactly commensurate with Si lattice. ${ }^{[7]}$ They both claimed the $1 \times 1$ phase co-exists with $\sqrt{ } 7 x \sqrt{ } 3$ phase. According to our experiments, the $1 \mathrm{x} 1$ phase may corresponds to $\sqrt{7} \mathrm{x} \sqrt{3}$ because our 1-D scan shows in $[11 \overline{2}]$ direction we have not only $103 \%$ (which agrees well with Bauer), but also $70 \%, 35 \%$ spots if we choose a different and proper energy $(60 \mathrm{eV})$. These spots are multiples of $\sqrt{ } 3 / 5$ and belong to $\sqrt{7} x \sqrt{ } 3$ phase. They are similar spots on $\mathrm{Pb} \sqrt{7} \mathrm{~V} \sqrt{3}$ phase observed before. ${ }^{[11]}$

$\mathrm{On} \mathrm{Pb} \sqrt{ } 7 \mathrm{x} \sqrt{3}$ phase, further small amount of deposition will produce "Devil's staircase(DS)". ${ }^{[12]}$ It will be interesting to study if we have similar effects on In surface. Some further depositions were made to verify if there is devil-staircase like similar experiments on $\mathrm{Pb} \sqrt{ } 7 \mathrm{x} \sqrt{3}$ phase. After $0.06 \mathrm{ML}$ of deposition, there are no the spots positions 
shift observed, which indicates there is no devil-staircase like $\mathrm{Pb}$. If there is $\mathrm{DS}$ then after these amount of deposition the peaks should move significantly.

2 comparisons between different phases

Comparing the experiments on the four In phases, the In $\beta$ phase and $\sqrt{3} 1$ are similar. They are both metastable phases. When In atoms are deposited, they will be converted to 1x1R30 phase before islands grow. In our experiments, the conversion happened at low temperature $(170 \mathrm{~K})$, which is not reported before. In previous studies, we only have reports that this phase transition was observed at higher temperatures (above $0{ }^{\circ} \mathrm{C}$ ). ${ }^{[8]}$ This discover indicates that Indium atoms are very mobile, and it can reconstruct its at as low as $170 \mathrm{~K}$, which is learned from previous experiments ${ }^{[15]}$. However, the conversion is not $100 \%$ that's why the intensity of most spots(fcc, $1 \mathrm{x} 1$ spot, etc.) grow on $\beta$ phase is lower than those directly grow on $1 \mathrm{x} 1 \mathrm{R} 30$.

In $4 \times 1$ is a stable phase. It doesn't convert into other phases with In depositions, and its structure keeps even at RT temperature. It is the 1x1R30 is the most preferred phase of the four and is more stable than $4 \times 1$ phase since both $\beta$-phase and $\sqrt{3} 1$ phase will convert to $1 \times 1$, not $4 \times 1$ phase.

3 comparisons with growth on $\mathrm{Pb}$ alpha phase

After the experiments on four different phases, we can see there are always In fcc island spots and no bet island spots in diffraction patterns, which is different from the In growth on $\mathrm{Pb}$ alpha phase. From STM experiment, we observed bct islands like in the In on $\mathrm{Pb}$ alpha phase experiment, but why we can't see their diffraction patterns? And we know bct structure is the bulk structure of In so it is the most stable structure, so it should not turn into other structure. The only reasonable explanation is that the bct islands grow too fast and becomes too big in short time so that it exceed the limit of the SPA-LEED. The limit of SPALEED is $0.3 \% \mathrm{BZ}$, which corresponding $\sim 100 \mathrm{~nm}$ in real space. In case the bct island becomes too huge, it is not possible to observe it from diffraction any more.

4 the mobility of In atoms 
Another approach to study islands is to study the $\mathrm{g}(\mathrm{s})$ curve using SPA-LEED ${ }^{[13]}$. (See Chapter.2 for details). In case there are certain dominate height on the surface, $g(s)$ curve can tell the height of islands. However, when we try to use this to study In depositions on In phases, it is proved not successful because there is no obviously out-of-phase condition. This can also explained by the extra mobility of indium atoms. The islands grow so fast that the distance between indium islands in short time exceeds the instrument' limit.

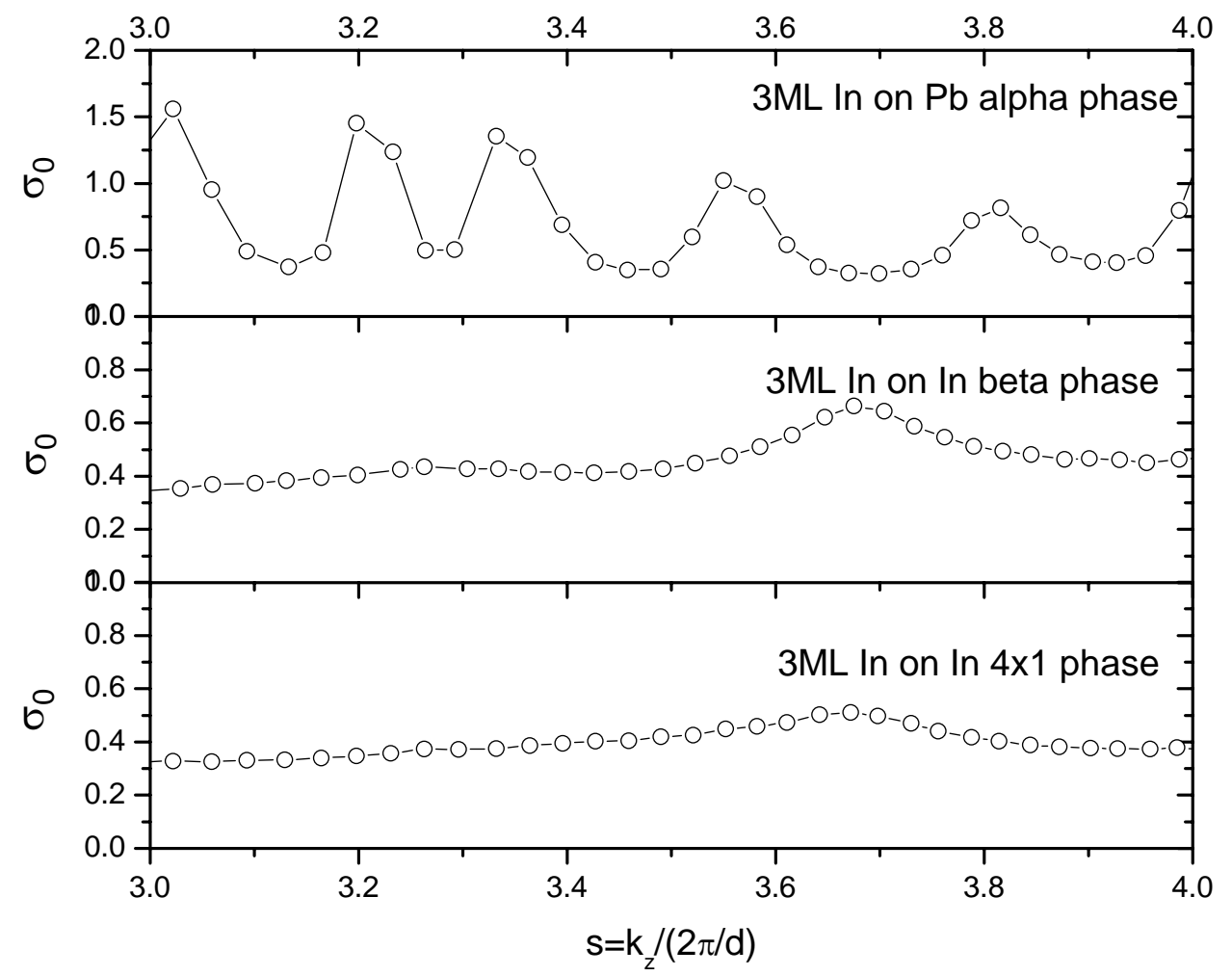

Figure $15 \sigma_{0} \sim S$ curve for In on three different phases.

Fig. 15 gives the $\sigma_{0} \sim \mathrm{s}$ curve for In islands grown on 3 different substrate- $\mathrm{Pb}$ alpha phase, In $\beta$ phase and In $4 \times 1$ phase. $s=\left(K_{z} /(2 \pi / d)\right)$ is scaled normal component momentum transfer and is function of electron energy. (see chp.2 for more details about $\mathrm{g}(\mathrm{s})$ curves) The $\sigma_{0}$ correspond to the width of (00) spot, its dependence on electron energy will reflect the property of In islands. We can tell on the $\mathrm{Pb}$ alpha phase, the value of $\sigma_{0}$ oscillating with energy and we can make the $\mathrm{g}(\mathrm{s})$ curve to get information from the surface. However, on the In beta and In $4 \times 1$ phases, the $\sigma_{0}$ values are always small, the maximum is just $0.6 \%$, which 
is already very close to the instrument limit $(0.3 \%)$. In this situation, it is impossible to get a good $g(s)$ curve. In physics, $\sigma_{0}$ corresponds to the size
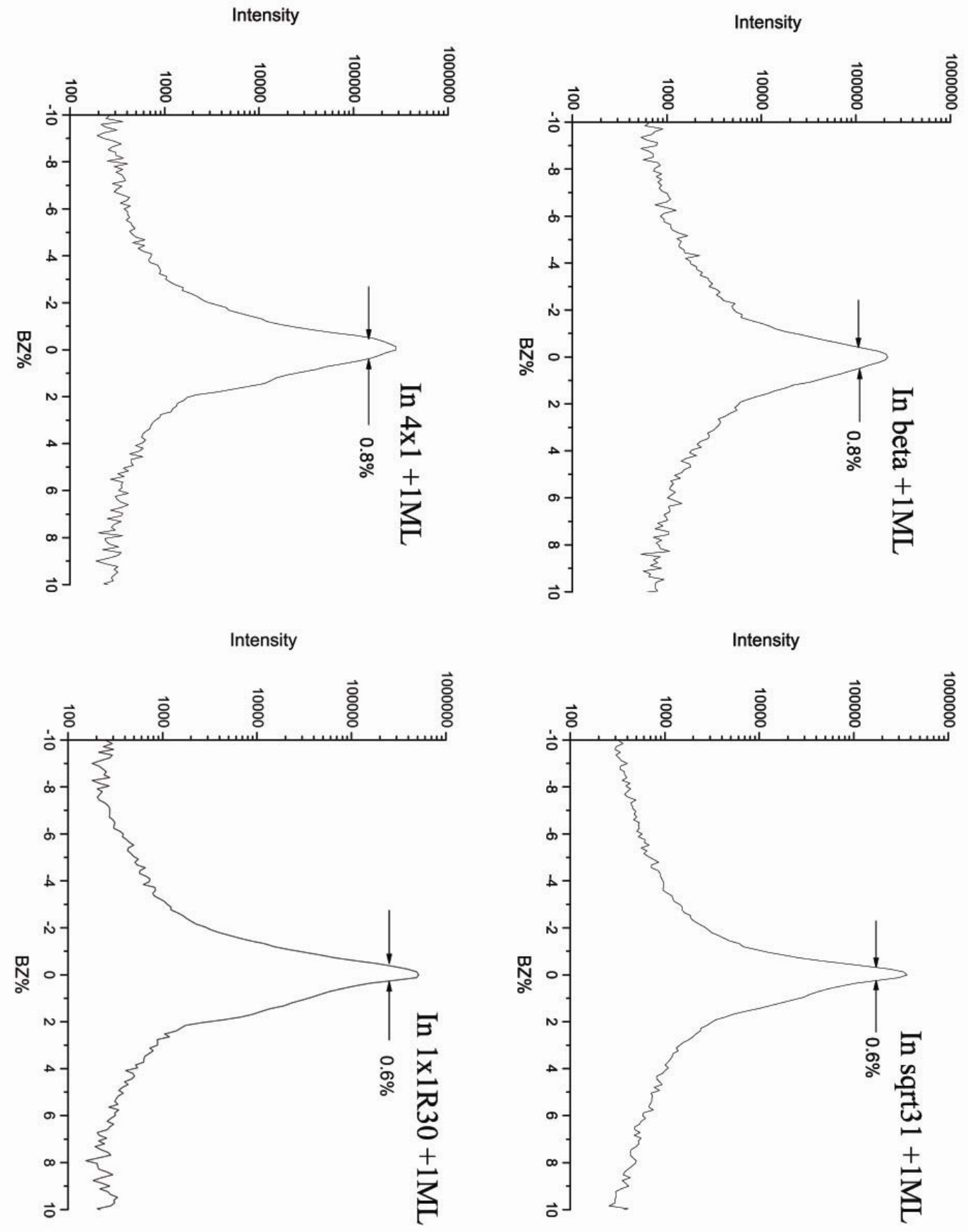

Figure 16 1-D profiles of (00)spot in[1 $1 \overline{1} 0]$ direction for the 4 different phases from SPA-LEED. The $\operatorname{FWHM}\left(\sigma_{0}\right)$ is marked in each curve.

of islands, small $\sigma_{0}$ means that the In islands are very large. 
Fig. 16 shows the 1-D profiles of (00) spot in [ $11 \overline{1} 0]$ direction for the 4 different In phases from SPA-LEED. In each of them we put $1 \mathrm{ML}$ of In on top at $\mathrm{T}=170 \mathrm{~K}$. The FWHM (full width of half maximum, or $\sigma_{0}$ in the $g(s)$ curve) of the (00) spot in these phases are all very small( $0.6-0.8 \%$, corresponding to $125-150 \mathrm{Si}$ unit cells or $48-60 \mathrm{~nm})$. The narrow peaks means that there are no uniform height islands on the surface or the islands are too big to be detected by the instruments. While when we put In on $\mathrm{Pb}$ alpha phase, $\sigma_{0}$ can be $1.5 \% \mathrm{BZ}$ in out-of-phase conditions (in fig.15).

Fig. 17 shows the huge difference of the $\mathrm{g}(\mathrm{s})$ curve taken for In on $\mathrm{Pb}$ alpha phase and In on In beta phase. In Fig.17(a) is the $\mathrm{g}(\mathrm{s})$ curve for the In on $\mathrm{Pb}$ alpha phase. It shows a clear 5-oscillation which indicates a 5-layer-island-dominate surface. In Fig.17(b) is the g(s) curve for the In on In beta phase. There is no any oscillation so we can't get useful information from it. This difference tells us that the indium islands are much more mobile on In substrate than on $\mathrm{Pb}$ substrate. Due to their mobility, the In islands grow so fast that the distance between indium islands in short time exceeds the instrument' limit.
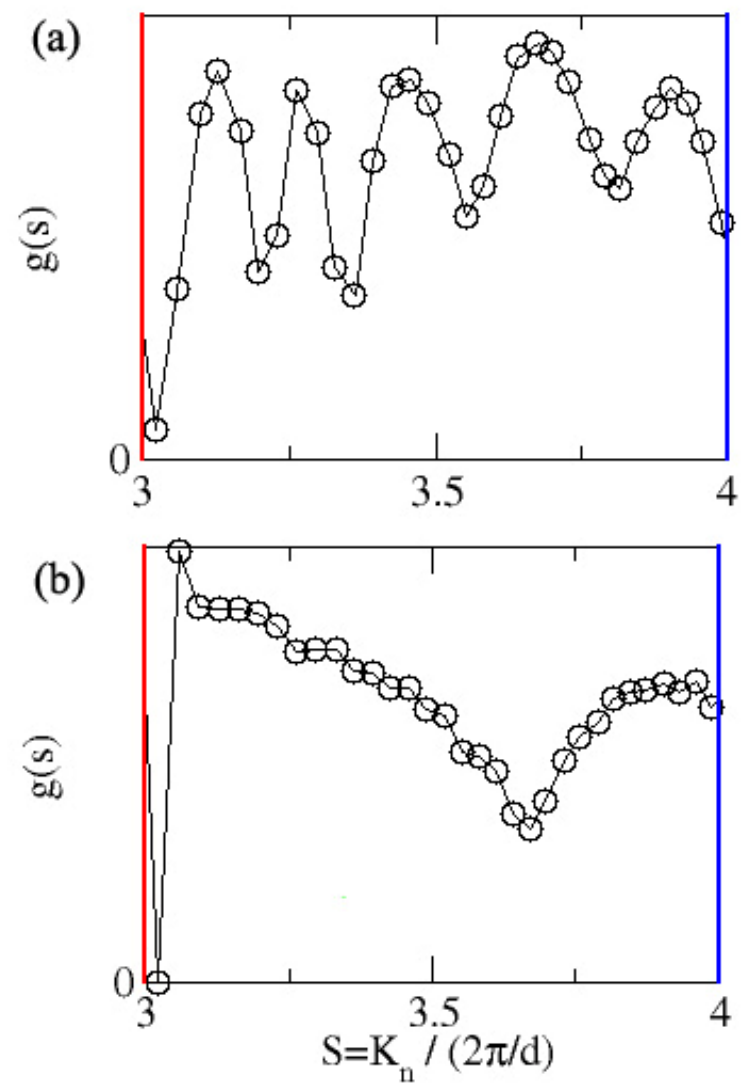

Figure 17 g(s) curve for (a) In on Pb alpha phase (b) In on In beta phase 
Several $g(s)$ curve are made for Indium deposition on different phase and in none of these $\mathrm{g}(\mathrm{s})$ curves we can see periodic oscillations which indicate uniform height islands. It not surprising because the (00) spot is always very narrow i.e. $\sigma_{0}$ is very small and out of the system's limit.

\section{Conclusion}

We studied Indium growth on varies of Indium phases at low temperature. We observed that the In beta phase and In $\sqrt{ } 31$ phase will convert into In 1x1R30 phase, which is not reported before. In $4 \times 1$ is a stable phase at low temperature as well as at room temperature. In the 4 phases, 1x1R30 phase is the most preferred. On all the In phase, In will grow fec islands at low temperature $(170 \mathrm{~K})$ and they becomes bet islands in a short time. These bet islands are very big and can not be observed from SPA-LEED due to the mobility

of In atoms. This experiment shows that In atoms are too mobiles on In substrate so $\mathrm{Pb}$ alpha phase is a special substrate that can grow In islands with QSE effects.

\section{References}

[1] J. J. Lander and J. Morrison, J. Appl. Phys. 36, 1706 1965(1964).

[2] H. Oefner, S. L. Surnev, Y. Shapira, and F. P. Netzer, Phys. Rev. B 48,10940 (1993).

[3] S. L. Surnev, J. Kraft, and F. P. Netzer, J. Vac. Sci. Technol. A 13, 1389 (1995).

[4] J. Kraft, S. L. Surnev, and F. P. Netzer, Surf. Sci. 340, 36 (1995).

[5] J. Kraft, M. Ramsey, and F. P. Netzer, Phys. Rev. B 55, 5384 (1997).

[6] C. Kumpf et al., Phys. Rev. Lett. 85, 4916 (2000).

[7] Pavlovska, E. Bauer and M. Giessen, J. Vac. Sci. Technol. B 20, 2478 (2002)

[8] Maojie Xu, et al., App. Phys. Lett., 94,073109(2009)

[9] A.A.Saranin, et.al, Surf. Sci. 450, 34(2000)

[10] J. Kraft, M.G. Ramsey, F.P. Netzer, Surf. Sci. 372, L271 (1997).

[11] M. Yakes, V. Yeh, M. Hupalo, and M. C. Tringides, Phys. Rev. B 69, 224103 (2004))

[12] M. Hupalo, J. Schmalian, and M.C. Tringides, Phys. Rev. Lett. 90, 216106 (2003). 
[13] S. Stepanovsky, M. Yakes, V. Yeh, M. Hupalo, M.C. Tringides, Surface Science 600 , 1417 (2006).

[14] J.Chen, M. Hupalo, M. Ji, C. Z. Wang, K. M. Ho, and M. C. Tringides, Phys. Rev. B 77, $233302(2008))$

[15] J.Chen, M. Hupalo, and M. C. Tringides, to be published. 


\section{CHAPTER 7: CONCLUSIONS}

Quantum size effect has attracted more and more interests in surface science due to many of its effects. One of its effects is the height preference in film growing and the resulting possibility of uniformly sized self-assemble nanostructure. The experiment of $\mathrm{Pb}$ islands on In $4 x 1$ phase shows that both the height and the width can be controlled by proper growth conditions, which expands the growth dimensions from 1 to 2 . This discover leads us to study the $\mathrm{In} / \mathrm{Pb}$ interface.

In Ch.3, we found that the $\mathrm{Pb}$ islands growing on $\mathrm{In} 4 \mathrm{x} 1-\mathrm{Si}(111)$ surface which have uniform height due to QSE and uniform width due to the constriction of In 4x1 lattice have unexpected stability. These islands are stable in even RT, unlike usual nanostructures on $\mathrm{Pb} / \mathrm{Si}$ surface which are stable only at low temperature. Since similar structures are usually grown at low temperature, this discovery makes the grown structures closer to technological applications. It also shows the unusual of $\mathrm{In} / \mathrm{Pb}$ interface.

Then we studied the In islands grown on $\mathrm{Pb}-\alpha-\sqrt{3} \mathrm{x} \sqrt{3}-\mathrm{Si}(111)$ phase in Ch.4. These islands have fcc structure in the first few layers, and then convert to bct structure. The In fcc islands have sharp height preference due to QSE like $\mathrm{Pb}$ islands. However, the preferred height is different (7 layer for $\mathrm{Pb}$ on $\mathrm{Si} 7 \mathrm{x} 7$ and 4 layer for $\mathrm{Pb}$ on $\mathrm{In} 4 \mathrm{x} 1$ ), due to the difference of interface. The In islands' structure prefers to be bct than fcc with coverage increase. It is quantitatively supported by first-principle calculation.

Unexpectedly, the In islands grown on various of In interfaces didn't show QSE effects and phase transition from fcc and bct structures as on the Pb- $\alpha$ interface (Ch.6). In $\mathrm{g}(\mathrm{s})$ curve there is no clear oscillations in the $\mathrm{g}(\mathrm{s})$ curve as the In on Pb- $\alpha$ phase. This may be due to the extra mobility of In atoms, which causes the In bct islands to grow too fast to be observed in diffraction or STM (Ch.5). From these experiments we can see the importance of $\mathrm{Pb}-\alpha$ phase in growth of In islands. It is the best interface to grow In islands in the phases we have experimented.

Recent experiments show that the Existence of $\mathrm{Pb}$ will decrease the diffusion speed of In. In Ch.6 we have shown that In atoms diffusion is so fast that the bct spots are not visible in diffraction. But when we put some $\mathrm{Pb}$ onto the In surface, we can see the bct spots, 
although very weak. So $\mathrm{Pb}$ should play an role in slowing down the indium atoms' diffusion. The interaction of $\mathrm{Pb}$ and In may play a role, but it is still not fully understood.

So the general conclusion of this thesis is that $\mathrm{In} / \mathrm{Pb}$ interface has extraordinary properties (e.g. special stability at RT, QSE effects.) and may have potential in selfassembling growth. There are unique effects on $\mathrm{Pb} /$ In interface which are not observed in pure $\mathrm{Pb}$ or In systems. And there is still no satisfactory theory that explains these effects. Many unsolved problems and further research is need. Why the $\mathrm{In} / \mathrm{Pb}$ interface is special? Is there a theory explanation? How does In and $\mathrm{Pb}$ interact with each other? Further research is needed to solve these problems. 


\section{ACKNOWLEDGMENTS}

I am very lucky to work with many outstanding scientists during my time at Iowa State University. Myron Hupalo has been a great source of guidance for me and is always ready to help when I have any trouble in the SPA-LEED and STM experiments. Steven Binz offered good suggestions and is willing to help in any time. Michael Yakes basically taught me everything I know about the SPA-LEED, and I thank him for many fruitful discussions. I also want to thank my adviser Michael Tringides for his guidance, encouragement and support. I would never finish this work without his help. Michael is not only an accomplished scientist but also one of the nicest people I have ever met.

Any success I have had or am going to have in my career and my life is a direct reflection on my family. I want to give my hearted thanks to my family: my father Qi Chen and my father Xiaolin Li. They are always patient and gave me priceless instruction and support throughout my life which will benefit me forever. 